\title{
Guidelines for Antibiotic Prescription in Intensive Care Unit
}

${ }^{1} \mathrm{GC}$ Khilnani, ${ }^{2}$ Kapil Zirpe, ${ }^{3}$ Vijay Hadda, ${ }^{4}$ Yatin Mehta, ${ }^{5}$ Karan Madan, ${ }^{6}$ Atul Kulkarni, ${ }^{7}$ Anant Mohan, ${ }^{8}$ Subhal Dixit, ${ }^{9}$ Randeep Guleria, ${ }^{10}$ Pradeep Bhattacharya, ${ }^{*}$ For the Expert Committee to formulate National Guidelines for Antibiotic Prescription in ICU

How to cite this article: Khilnani GC, Zirpe K, Hadda V, Mehta Y, Madan K, Kulkarni A, Mohan A, Dixit S, Guleria R, Bhattacharya P. Guidelines for Antibiotic Prescription in Intensive Care Unit. Indian Journal of Critical Care Medicine 2019;23(Suppl 1): S1-S63.

\section{Source of support: Nil}

Conflict of interest: None

\section{EXECUTIVE SUMMARY}

\section{Pharmacokinetics and Pharmacodynamics}

\section{Evidence Statement}

Time-dependent antibiotics require drug concentrations greater than the minimum inhibitory concentration (MIC) for a certain period between doses, which usually ranges from 40 to $50 \%$ of the inter-dose interval for their best action. Continuous infusions are preferred over extended infusions for beta-lactam antibiotics and are associated with clinical benefits like a decrease in hospital stay, cost of therapy and mortality. For vancomycin, continuous infusion is associated with reduced toxicity and cost of therapy but no mortality benefit.

\section{COMMUNITY-ACQUIRED PNEUMONIA IN THE INTENSIVE CARE UNIT}

\section{What are the Common Organisms Causing Community-Acquired Pneumonia in Intensive Care Unit Worldwide and India?}

\section{Evidence Statement}

Streptococcus pneumoniae, gram-negative bacilli (including klebsiella, Haemophilus influenzae), atypical organisms (Mycoplasma pneumoniae) and viruses (including influenza) are common causes of community-acquired pneumonia (CAP) in intensive care unit (ICU). Staphylococcus aureus, Legionella, and Mycobacterium tuberculosis are less common causes of CAP in ICU. Pseudomonas aeruginosa is an important pathogen causing CAP in patients with structural

Corresponding Author: GC Khilnani, Professor and Head, Department of Pulmonary Medicine and Sleep Disorders, All India Institute of Medical Sciences, New Delhi, India, Phone: 01129593488, e-mail: gckhil@gmail.com lung disease. Methicillin-resistant Staphylococcus aureus (MRSA) and multidrug-resistant gram-negative organisms are relatively infrequent causes of CAP in India and are associated with risk factors such as structural lung disease and previous antimicrobial intake. Anaerobic organisms may cause CAP or co-infection in patients with risk factors for aspiration like elderly, altered sensorium, dysphagia, head, and neck malignancy. S. pneumoniae remains sensitive to beta-lactams and macrolides. Haemophilus influenzae has good sensitivity to beta-lactam with beta-lactamase inhibitors and fluoroquinolones. Recent studies show an increasing prevalence of extended spectrum $\beta$-lactamase (ESBL) producing Enterobacteriaceae.

\section{What are the Risk Factors For Multidrug- Resistant (MDR) Pathogens for CAP In ICU?}

\section{Evidence Statement}

Risk factors for multidrug-resistant (MDR) organisms include age $>65$ years, antimicrobial therapy in the preceding 3 months, high frequency of antibiotic resistance in the community, hospitalization for $\geq 48$ hours in the preceding 3 months, home infusion therapy including antibiotics, home wound care, chronic dialysis within 1 month, family member with MDR pathogen and ongoing immunosuppressive treatment.

\section{Recommendations}

- All patients admitted with CAP in ICU should be evaluated for risk factors for infection with MDR organisms (2A).

- Antibiotic therapy should be individualized to cover the commonly implicated organisms according to risk factors, including Pseudomonas, ESBL producing Enterobacteriaceae or MRSA (3A).

\section{How Early Should the Antibiotics be Initiated in} Patients with CAP Who Require ICU Admission?

\section{Evidence Statement}

Early initiation of antibiotics has been associated with a reduction in all-cause mortality in community-acquired

*Expert Committee: Arti Kapil, Pawan Tiwari, Saurabh Mittal, Dhruva Chaudhary, JC Suri, MK Daga, Yash Zaveri, Suresh Rama Subban, Seema Sood, RK Mani, Narendra Rungta, Anirban Chaudhry, Rajesh Pandey, Neetu Jain, Arvind Baronia, Jaya Kumar, Gyanendra Agarwal, Camilla Rodrigues, BK Rao, Deepak Govil, Sachin Gupta, Ashit Hegde, Pramod Garg, Sandeep Mahajan, Chand Wattal, Rajesh Chawla, Anjan Trikha, Prakash Shastri, Anil Gurnani, Rajesh Mishra, Rohit Bhatia, GC Khilnani, Kapil Zirpe, Vijay Hadda, Anant Mohan, Atul Kulkarni, Karan Madan, Yatin Mehta, Subhal Dixit, Randeep Guleria, Pradeep Bhattacharya 
pneumonia, including severe pneumonia with sepsis or septic shock.

\section{Recommendations}

- Appropriate antimicrobial therapy should be initiated as early as possible in patients of CAP requiring ICU admission, preferably within the first hour after obtaining necessary microbiologic samples (3A).

\section{Should CAP in ICU Receive Empirical Antimicrobials or Upfront Targeted Antimicrobial Therapy?}

\section{Evidence Statement}

Early institution of targeted antibiotic therapy in severe CAP based on urinary antigen testing is associated with a higher relapse rate without any mortality benefit in prospective randomized studies. Retrospective studies have shown mortality benefit with narrowing down of antibiotic therapy based on results from cultures of respiratory specimens, blood cultures as well as Legionella and pneumococcal urinary antigen testing.

\section{Recommendations}

- Empirical therapy covering common etiologic organisms should be initiated for severe CAP requiring ICU admission (2A).

- Investigations including the culture of respiratory secretions (sputum, endotracheal aspirate), blood cultures, urinary antigen testing for Pneumococcus and Legionella may be performed to narrow down therapy. Bronchoscopic BAL or protected specimen brush samples or polymerase chain reaction (PCR) for viral etiology may be performed for microbiologic diagnosis on a case by case basis (3A).

For Empirical Therapy in Patients with CAP in ICU, Should Combination Therapy be Preferred Over Monotherapy?

\section{Evidence Statement}

Empirical combination therapy covering common organisms causing community-acquired pneumonia improves survival without any significant increase in microbial resistance.

\section{Recommendations}

- Patients with CAP requiring ICU admission should initially receive a combination of empirical antimicrobial agents covering common causative organisms (2A).

\section{What Should be the Preferred Combination Therapy for CAP in ICU?}

\section{Evidence Statement}

For patients with severe CAP requiring ICU admission without risk factors for pseudomonal infection, a combination of beta-lactams along with macrolides is better as compared to beta-lactam fluoroquinolone combination in terms of mortality benefit and length of hospital stay.

\section{Recommendations}

- For patients with CAP requiring ICU admission, a non-pseudomonal beta-lactam (cefotaxime, ceftriaxone, or amoxicillin-clavulanic acid) plus a macrolide (azithromycin or clarithromycin) should be preferred if there are no risk factors for Pseudomonas aeruginosa infection (1A).

- For penicillin-allergic patients, a respiratory fluoroquinolone (levofloxacin, moxifloxacin or ciprofloxacin) and aztreonam may be used (3A).

- If macrolides cannot be used, a fluoroquinolone may be used if there is no clinical suspicion of tuberculosis, after sending sputum or endotracheal aspirate for AFB and Genexpert (3A).

\section{When Should Anti-Pseudomonal Cover be Added for CAP in ICU? If Required, Which are the Preferred Antimicrobials for Anti- Pseudomonal Cover?}

\section{Evidence Statement}

For patients with severe CAP requiring ICU admission, risk factors for infection with Pseudomonas aeruginosa include chronic pulmonary disease (chronic obstructive pulmonary disease, asthma, bronchiectasis), frequent systemic corticosteroid use, prior antibiotic therapy, old age, immunocompromised states, enteral tube feeding, cerebrovascular or cardiovascular disease. Prior antibiotic therapy is a risk factor for multidrug-resistant pseudomonal infection.

\section{Recommendations}

- If $P$. aeruginosa is an etiological consideration, antipneumococcal, antipseudomonal antibiotic (like ceftazidime, cefoperazone, piperacillin-tazobactam, cefoperazone-sulbactam, imipenem, meropenem or cefepime) should be used (2A).

- Combination therapy should be considered with the addition of aminoglycosides or antipseudomonal fluoroquinolones (e.g., ciprofloxacin) (3A). 


\section{When Should MRSA Cover be Added to the Empiric Regimen for CAP in ICU?}

\section{Evidence Statement}

Risk factors for MRSA in CAP in ICU include close contact with MRSA carrier or patient, influenza, prisoners, professional athletes, army recruits, men having sex with men (MSM), intravenous (IV) drug abusers, regular sauna users and those with recent antibiotic use. MRSA pneumonia should be suspected after influenza or in previously healthy young patients, if there is cavitation or necrotizing pneumonia, along with rapid increase of pleural effusion, massive hemoptysis, neutropenia or erythematous rashes. Vancomycin, teicoplanin, linezolid, and tigecycline are effective antibiotics against MRSA.

\section{Recommendations}

- All patients admitted with CAP in ICU should be evaluated for the presence of risk factors associated with MRSA (3A).

- If MRSA is a consideration, empiric vancomycin (1A) or teicoplanin (2A) should be added to the regimen. Linezolid should be used for vancomycin intolerant patients, vancomycin-resistant Staphylococcus aureus (VRSA), or patients with renal failure (1A).

\section{When Should Anaerobic Cover be Added to the Empiric Antibiotic Regimen for CAP in ICU?}

\section{Evidence Statement}

Risk factors for aspiration pneumonia in patients admitted with CAP in ICU include dysphagia, altered sensorium, coma, witnessed aspiration, putrid discharge, the presence of lung abscess, empyema or necrotizing pneumonia.

\section{Recommendations}

- Empirical antibiotics with anaerobic coverage should be considered in the treatment of CAP in ICU in the presence of clinical risk factors for aspiration or presence of lung abscess, empyema or necrotizing pneumonia(2A).

\section{Which Antibiotic Should be Preferred for Anaerobic Coverage for CAP in ICU?}

\section{Evidence Statement}

Commonly prescribed empirical antibiotics for CAP in ICU such as ampicillin-sulbactam, amoxicillin-clavulanic acid, piperacillin-tazobactam, and carbapenems have excellent anaerobic coverage. Clindamycin and moxifloxacin are effective against aspiration and lung abscess caused by anaerobic organisms. Lung abscess and necrotizing pneumonia may require prolonged treatment up to 4 to 6 weeks.

\section{Recommendations}

- Patients with CAP at risk of anaerobic infection should be initiated on antibiotics with anaerobic activity such as amoxicillin-clavulanate, clindamycin or moxifloxacin (1A).

- Piperacillin-tazobactam or carbapenems can be used for empirical therapy in CAP due to anaerobes if otherwise indicated (3A).

- Duration of treatment should be individualized according to the response and severity of the disease (3A).

\section{What Should be the Optimal Duration of Antibiotics for CAP in ICU?}

\section{Evidence Statement}

For CAP in ICU, there is limited evidence regarding the duration of treatment, with no significant mortality benefit beyond 7 days of antimicrobial therapy in uncomplicated cases. However, CAP due to GNB, Enterobacteriaceae, $P$. aeruginosa, $S$. aureus bacteremia, and L. pneumophila requires prolonged treatment. Necrotizing pneumonia, lung abscess, empyema or extrapulmonary infective complications like meningitis or infective endocarditis also require a longer duration of treatment.

\section{Recommendations}

- Patients with CAP requiring ICU admission should receive antibiotics for 7 to 10 days (2A).

- Patients with CAP due to Pseudomonas or aspiration pneumonia should be treated for 14 days (3A).

- Necrotizing pneumonia due to GNB, MRSA or anaerobes also require treatment for 14 to 21 days (3A)

- Duration of treatment should be individualized according to causative organism, response, the severity of disease and complications (3A).

\section{Should Procalcitonin be used to Determine the Duration of Antibiotic Administration for CAP in ICU?}

\section{Evidence Statement}

Serial procalcitonin levels can be used for de-escalation of antibiotics for CAP in ICU, without any increase in mortality or recurrence rates.

\section{Recommendations}

- Procalcitonin levels can be used along with clinical judgment for de-escalation of antibiotics in CAP in ICU in patients treated beyond 5 to 7 days (1A). 


\section{VENTILATOR ASSOCIATED PNEUMONIA}

\section{What are the Common Organisms Causing HAP/VAP in ICU and What is their Antibiotic Susceptibility Pattern?}

\section{Evidence Statement}

Ventilator-associated pneumonia (VAP) and hospitalacquired pneumonia (HAP) are commonly caused by aerobic gram-negative bacilli, such as Acinetobacter baumannii, Klebsiella pneumoniae, Pseudomonas aeruginosa, or by gram-positive cocci (Staphylococcus aureus).In Indian ICUs, gram-negative organisms are the most common etiologic agents (i.e., Acinetobacter, Klebsiella and Pseudomonas spp). Most of these pathogens have been found to be multidrug resistant. The frequency of specific MDR pathogens causing HAP and VAP may vary by hospital, patient population, type of ICU patient, and change over time.

\section{What are the Risk Factors for MDR Pathogens in VAP in ICU?}

\section{Evidence Statement}

The risk factors for VAP due to MDR organisms include age $>60$ years, duration of mechanical ventilation $\geq 7$ days, prior antibiotic use within 3 months, the presence of severe sepsis or septic shock at the time of VAP, ARDS preceding VAP, renal replacement therapy before VAP and systemic corticosteroid therapy.

\section{What Should be the Initial Combination of Empiric Antibiotic Therapy for VAP in ICU?}

\section{Evidence Statement}

Use of antibiotic monotherapy and combination therapy for VAP have similar outcomes in patients who are not at risk for MDR pathogens. Commonly used antimicrobial agents include piperacillin-tazobactam, cefepime, levofloxacin, imipenem, and meropenem. Among antimicrobial agents, carbapenems have a higher chance of clinical cure than non-carbapenems. For treatment of VAP due to MRSA, glycopeptides and linezolid have similar clinical success; however, linezolid may be associated with a higher chance of thrombocytopenia and gastrointestinal adverse events.

\section{Recommendations}

- Among patients with VAP who are not at high risk of MDR pathogens and are in ICUs with a low prevalence of MRSA $(<15 \%)$ and resistant gram-negative organisms $(<10 \%)$, single antibiotic active against both MSSA and Pseudomonas is preferred over combination antibiotic (1A)
- Among patients with VAP who are at high risk of MDR pathogens or are in ICU with a high prevalence of MRSA $(>15 \%)$ and resistant gram-negative organisms $(>10 \%)$, an agent active against MRSA and at least two agents active against gram-negative organisms including $P$. aeruginosa is recommended (3A)

- Among patients with VAP who are not at high risk of MDR pathogens and are in ICU with a high prevalence of resistant gram-negative organisms ( $>15 \%)$ but low prevalence of MRSA $(<10 \%)$, two agents active against gram-negative organism including $P$. aeruginosa is recommended (3A)

- Colistin is not recommended for routine use as an empirical agent in VAP. However, it may be used upfront in the ICUs if there is a high prevalence of carbapenem-resistant Enterobacteriaceae (> 20\%) (UPP).

- In our country or areas with high endemicity of tuberculosis, use of linezolid may be restricted unless no suitable alternative is available (UPP).

- Fluoroquinolones and aminoglyosides should be cautiously used as monotherapy in VAP in our country as well as in other areas with high endemicity of tuberculosis (UPP).

- In ICUs where the distribution of pathogen and antibiotic resistance pattern is known, empiric treatment should be designed accordingly, based upon patient risk factors for MDR pathogens. (UPP)

\section{When to give Antipseudomonal Drugs for VAP in ICU?}

\section{Evidence Statement}

Prior use of antibiotics (most consistent association), prolonged duration of mechanical ventilation, and chronic obstructive pulmonary disease (COPD) have been identified as risk factors for MDR P. aeruginosa infection.

\section{Recommendations}

- Empiric treatment should be given to cover Pseudomonas if there are risk factors for MDR Pseudomonas infection (2A).

- In ICUs where gram-negative isolate resistance rate is low $(<10 \%$ gram-negative isolate resistant to the agent being considered for monotherapy) and patients have no risk factors for antimicrobial resistance, one antipseudomonal antibiotic may be given (3A).

- In ICUs where gram-negative isolate resistance rate is high (>10\% gram-negative isolate resistant to the agent being considered for monotherapy or not known), two anti-pseudomonal antibiotics from a different class to be given (3A). 


\section{What Should be the Duration of Antibiotic Treatment for HAP/VAP?}

\section{Evidence Statement}

Short-course regimens for VAP are associated with significantly more antibiotic-free days without any significant difference in the duration of ICU or hospital stay, recurrence of VAP and mortality. Short-course regimens are associated with more recurrences in VAP due to non-fermenting gram-negative bacilli (NF-GNB).

\section{Recommendations}

- Short course (7-8 days) of antibiotic therapy should be used, in the case of VAP with good clinical response to therapy $(1 \mathrm{~A})$.

- Longer duration (14 days) of antibiotic therapy should be considered, in case of VAP caused by NF-GNBs or is associated with severe immunodeficiency, structural lung disease (COPD, bronchiectasis, and interstitial lung disease), empyema, lung abscess, necrotizing pneumonia, and inappropriate initial antimicrobial therapy (3A).

\section{When Should Anaerobic Cover be Added for VAP and Which is the Preferred Antimicrobial Agent?}

\section{Evidence Statement}

The incidence of anaerobic bacteria as the causative agent of VAP is 2 to $7 \%$. Risk factors for VAP due to anaerobes are altered consciousness, aspiration pneumonitis and high simplified acute physiology score (SAPS).

\section{Recommendations}

- Empirical antibiotic regimen for VAP should not include coverage for anaerobic organisms routinely (2A).

- In the presence of risk factors for VAP due to anaerobic pathogens, anaerobic antimicrobial coverage should be added in an empirical regimen (2B).

- In patients with risk factors for anaerobic organisms, clindamycin or metronidazole should be added to empirical antibiotics regimen for VAP, if it does not include carbapenems (meropenem or imipenem) or piperacillin-tazobactam in the ongoing empirical regimen (UPP).

\section{When to Give Atypical Cover for VAP and Which is the Preferred Agent?}

\section{Evidence Statement}

The incidence of atypical bacteria as causative agents of VAP is low (5 to 7.5\%). Risk factors for VAP due to Legionella are Legionella colonization in hospital water supply, prolonged use of corticosteroids, cytotoxic chemotherapy, elderly, chronic renal failure, previous antibiotic use, granulocytopenia, and poor Glasgow coma score.

\section{Recommendations}

- Empirical antibiotic regimen for VAP should not include coverage for atypical organisms routinely (2A).

- In the presence of risk factors for VAP due to atypical bacterial pathogens, atypical antimicrobial coverage should be added to the empirical regimen (2B).

- The preferred atypical coverage in combination antibiotics regimen is fluoroquinolones (levofloxacin or moxifloxacin) or macrolides (azithromycin or clarithromycin) (UPP).

\section{Can Serum Procalcitonin be used for De-escalation of Antibiotic Therapy in VAP?}

\section{Evidence Statement}

Use of procalcitonin to guide de-escalation of antibiotic treatment in patients with VAP is effective in reducing antibiotic exposure, without an increase in the risk of mortality or treatment failure.

\section{Recommendations}

- Serum procalcitonin may be used to guide the de-escalation of antibiotics in VAP when the anticipated duration of therapy is $\geq 7$ to 8 days (1B).

- Serum procalcitonin levels (together with clinical response) should be used for de-escalation of antibiotic therapy in VAP in specific clinical conditions (severely immunocompromised patients, drug-resistant pathogens-NF-GNB, initial inappropriate therapy) (3A).

\section{How to Approach a Patient of Non-responding VAP?}

\section{Evidence Statement}

Re-evaluation at 48 to 72 hours after the initial diagnosis of VAP is the most suitable time. By then the results of the initial microbial investigation are usually available, and treatment modification can be done. Evaluation of treatment response for VAP should be on the basis of clinical, laboratory, radiograph and microbiological results. Factors associated with treatment failure in VAP includes host factors (advanced age, immunosuppressed, chronic lung disease, ventilator dependence), bacterial factors (drug-resistant pathogens, opportunistic pathogens), therapeutic factors (inappropriate antibiotics, delayed initiation of therapy, insufficient duration of therapy, suboptimal dosing, inadequate local concentration of drugs), complications of initial VAP episode (lung 
abscess, empyema), other non-pulmonary infections or non-infectious mimics of pneumonia.

\section{Recommendations}

- Non-responding VAP should be evaluated for noninfectious mimics of pneumonia, unsuspected or drug-resistant pathogens, extrapulmonary sites of infection, and complications of pneumonia or its therapy and diagnostic testing should be directed to whichever of these causes is likely (2A).

\section{CATHETER RELATED BLOODSTREAM INFECTIONS (CRBSI)}

\section{What is the Incidence of Catheter Colonization and CRBSI?}

\section{Evidence Statement}

The global incidence of CC ranges from 1.4 to $19.4 \%$ whereas CRBSI incidence ranges from 2.4 to $12.5 \%$. The incidence of CC is higher in Indian ICUs ranging from $18 \%$ to as high as $59 \%$, whereas the incidence of CRBSI is up to 16.1 per 1000 catheter days.

\section{What are the Risk Factors for CRBSI?}

\section{Evidence Statement}

Longer indwelling catheter duration, immunosuppression, diabetes mellitus, sepsis at the time of insertion, multi-lumen catheters and APACHE $>23$ are important risk factors for CRBSI. APACHE at admission, renal failure, central venous catheterization, and steroid therapy are important risk factors for fungal CRBSI.

\section{What are the Common Organisms Causing CRBSI and their Antibiotic Susceptibility?}

\section{Evidence Statement}

Coagulase-negative staphylococci (CONS), S. aureus, Enterococcus, and Candida species are the common organisms accounting for the majority of the CRBSIs. A large proportion of Staphylococcus aureus and CONS are methicillin resistant ranging from 11 to $87 \%$. There is an increased incidence of CRBSI due to gram-negative organisms (most of which are ESBL producers) and candida especially the non-albicans candida.

\section{What is/are the Empiric Antibiotic(s) of Choice for CRBSI in ICU?}

\section{Evidence Statement}

Vancomycin, teicoplanin, linezolid, and daptomycin are effective in the treatment of CRBSI due to MRSA and MR-CONS. Fourth-generation cephalosporins, carbapenems or beta-lactam/beta-lactamase combination like piperacillin/tazobactam and aminoglycosides might be used for gram-negative organisms causing CRBSI. Caspofungin and fluconazole are equally effective as amphotericin-B for treatment of candidemia.

\section{Recommendations}

- Empirical antibiotic regimen for CRBSI should include coverage for both gram-positive and gram-negative organisms (2A).

- Vancomycin or teicoplanin is the recommended firstline drug for the empiric treatment of CRBSI for MRSA and MR-CONS while linezolid and daptomycin are good alternative agents (2A).

- Empiric coverage for gram-negative bacilli should include a fourth-generation cephalosporin, a carbapenem, or a $\beta$-lactam $/ \beta$-lactamase inhibitor combination, with or without an aminoglycoside (UPP).

- An echinocandin or fluconazole should be used as empirical antifungal agents for the treatment of suspected central line-associated candidemia (2A).

\section{What Should be the Duration of Antibiotic Treatment for CRBSI?}

\section{Evidence Statement}

Short duration ( $<14$ days) of antibiotics is as effective as longer duration ( $>14$ days) for uncomplicated Staphylococcus aureus bacteremia. Complicated bacteremia due to $S$. aureus or those associated with endocarditis should receive longer duration. For gram-negative bacteremia, seven days of antibiotics are sufficient. In responding patient with uncomplicated CONS infection, 5 to 7 days therapy is considered optimum. Minimum 14 days treatment with antifungals is required for fungal CRBSI.

\section{Recommendations}

- Minimum 2 weeks antibiotics should be given for uncomplicated and 4 to 6 weeks for complicated Staphylococcus aureus CRBSI and infective endocarditis (2A).

- Minimum 7 days of antibiotics should be given for gram-negative CRBSI (2A).

- Five to seven days antibiotics are recommended for CONS bacteremia (3A).

- For suspected fungal CRBSI, antifungal therapy for at least 14 days is recommended (UPP). 
Guidelines for Antibiotic Prescription in Intensive Care Unit

\section{URINARY AND UROGENITAL SEPSIS IN ICU}

What is the Incidence of UTI in ICU? What are the
Common Organisms and Risk Factors for UTI in ICU?

\section{Evidence Statement}

The incidence of CA-UTI ranges from $5-30 \%$ of all ICU admissions. The most common organism causing UTI in ICU are gram-negative bacteria (E. coli, Klebsiella) and fungi (especially Candida). Risk factors for UTI in ICU include the duration of catheterization, length of ICU stay, prior antibiotic use, higher disease severity score, and female gender.

\section{What is the Empirical Antimicrobial Agent of Choice for Treating UTI in ICU?}

\section{Evidence Statement}

There has been a trend towards increasing prevalence of extended-spectrum beta-lactamase producing gramnegative bacteria in the urinary cultures of catheterassociated UTI. Aminoglycosides, beta-lactams along with a beta-lactamase inhibitor as well as carbapenems and fosfomycin have good efficacy in catheter-associated UTI. The susceptibility for fluoroquinolones is decreasing over time among organisms isolated from nosocomial UTI. Candida species isolated from the patients with UTI show sensitivity to fluconazole.

\section{Recommendations}

- The initial choice of antibiotics should cover for ESBL producing gram-negative organisms and includes aminoglycosides, beta-lactam along with a beta-lactamase inhibitor or carbapenems (2A).

- In the initial empirical regimen for UTI, antibiotics against gram-positive organisms are not recommended (3A).

- In appropriate clinical settings, antifungals should be considered in the empirical regimen (3B).

\section{ACUTE INFECTIVE DIARRHEA, ANTIBIOTIC INDUCED DIARRHEA, AND CLOSTRIDIUM DIFFICILE ASSOCIATED DIARRHEA}

\section{What are the Common Organisms Causing Acute Infective Diarrhea in the ICU?}

\section{Evidence Statement}

The incidence of diarrhea in the ICU ranges from 12.9 to $38 \%$. Majority of the cases of diarrhea in ICU are noninfectious in etiology. Clostridium difficile is responsible for the majority of infectious cases of diarrhea in ICU.
What are the Empirical Antibiotics of Choice for Treating Acute Infective Diarrhea in the Icu?

\section{Evidence Statement}

Empirical use of metronidazole in patients with diarrhea suspected due to Clostridium difficile in ICU setting results in significant symptomatic improvement.

\section{Recommendations}

- We recommend that empiric metronidazole be used for therapy of patients with acute diarrhea in the ICU with suspected Clostridium difficile infection (3A).

\section{What are the Risk Factors for the Development of CDI or CDAD?}

\section{Evidence Statement}

Risk factors for the development of CDI include prior antibiotic therapy, advanced age, prolonged ICU/hospital stay, immunosuppression, proton pump inhibitors, and enteral feeding. Cephalosporins, clindamycin, fluoroquinolones, carbapenems, and penicillin derivatives are the commonly implicated antibiotics for CDAD/ CDI.

\section{What is the Recommended Treatment for CDI/CDAD: Which Antibiotics and Duration? Should Offending Antibiotics be Stopped? What is the Role of Probiotics in the Treatment of CDAD? How should Recurrent Clostridium difficile Infection be Treated?}

\section{Evidence Statement}

Both metronidazole and oral vancomycin have similar efficacy in the clinical and bacteriologic cure of CDI. Use of implicated antibiotic after completing the treatment of CDI is associated with increased risk of recurrence of CDI. There is insufficient evidence to justify the use of probiotics as an adjunct to antibiotics in the treatment of CDAD. In a single RCT, fecal microbiota transplantation was found to be highly efficacious for treatment of recurrent CDI.

\section{Recommendations}

- We recommend metronidazole as the first line treatment of mild to moderate CDI/CDAD (1A).

- We recommend oral vancomycin as the first line treatment of microbiologically proven severe CDI/CDAD (1A). 
- We recommend oral vancomycin as the treatment of recurrent $\mathrm{CDI} / \mathrm{CDAD}$ infection $(2 \mathrm{~A})$.

- We recommend fecal microbiota transplantation as an alternate treatment of recurrent CDI/CDAD infection (2A).

- We recommend that implicated antibiotics should be discontinued as soon as clinically feasible (2A).

- We recommend against the use of probiotics as an adjunct for the treatment of CDI/CDAD (2A).

- We recommend the addition of vancomycin to a patient with microbiologically proven CDI/CDAD if the patient is already on metronidazole or has no clinical response to metronidazole within 3 to 4 days (UPP)

\section{ABDOMINAL INFECTIONS IN ICU}

\section{Acute Pancreatitis and Infected Pancreatic Necrosis}

\section{What is the Incidence, risk factors, and microbiology of pancreatic infection following acute pancreatitis?}

\section{Evidence Statement}

The incidence of pancreatic infection following acute pancreatitis ranges from 12 to $37 \%$. Presence of pancreatic necrosis of $>50 \%$ is a major risk factor for pancreatic infection following acute pancreatitis. Primary organ failure predicts the development of infective pancreatic infection in patients with acute pancreatitis.

Gram-negative organisms are the most common organisms isolated from infected pancreatic necrosis following acute pancreatitis in Indian patients. Prophylactic antibiotic use in patients of AP to prevent IPN has been associated with increased risk of infection with gram-positive organisms. Resistance to carbapenems, beta-lactam/beta-lactamase inhibitors and quinolones in gram-negative organisms isolated from IPN has increased, however, with maintaining sensitivity to colistin and tigecycline.

\section{What are the Empirical Antibiotics of Choice for Treatment of Pancreatic Infection Following Acute Pancreatitis?}

\section{Evidence Statement}

Prophylactic use of antibiotics in patients with necrotizing pancreatitis has not been shown to reduce the incidence of pancreatic infection and mortality. Presence of persistent fever, leucocytosis, multiorgan failure and presence of air within pancreatic necrosis suggest infected pancreatic necrosis. Cephalosporins, piper- acillin-tazobactam, quinolones, and carbapenems have the highest whereas aminoglycosides have the lowest penetration into necrotic pancreatic tissue. Response to antibiotic therapy is assessed by clinical and radiological parameters.

\section{Recommendations}

- Routine use of prophylactic antibiotics to prevent pancreatic infection following acute pancreatitis of any severity is not recommended (1A)

- Empirical antibiotic regimen in patients with infected pancreatic necrosis should be guided by local microbiological data, susceptibility pattern, the pharmacokinetic property of antibiotics and previous antibiotic exposure (UPP).

- In treatment-naïve patients with evidence of infected pancreatic necrosis, we recommend empirical treatment with either carbapenems, piperacillin-tazobactam or cefoperazone- sulbactam (2A).

- In patients not responding or already exposed to the piperacillin-tazobactam, cefoperazone- sulbactam or carbapenems, colistin should be added to the empirical regime. (3B)

- Duration of antibiotic therapy should be guided by clinical, radiological and laboratory parameters (UPP).

- Patients not responding to antibiotics should undergo necrosectomy and drainage (3B).

\section{BILIARY SEPSIS}

\section{Acute Cholangitis}

\section{What is the Incidence, Risk Factors and Microbiology of Biliary Infection in ICU? What are the Empirical Antibiotics of Choice for Treatment of Biliary Infections in ICU?}

\section{Incidence and risk factors}

\section{Evidence Statement}

The incidence of acute cholangitis varies with underlying etiology and ranges from 0.2 to $10 \%$. Cholelithiasis, choledocholithiasis, benign and malignant common bile duct (CBD) strictures, CBD interventions and stenting are the most common risk factors for cholangitis.

\section{Microbiology of Acute Cholangitis}

\section{Evidence Statement}

Gram-negative organisms are the most common organisms isolated from patients with acute cholangitis. Most of the pathogens isolated are susceptible to third-generation cephalosporins (such as cefoperazone-sulbactam), 
aminoglycosides, quinolones, ureidopenicillins, and carbapenems. Risk factors for multidrug drug resistant organisms causing acute cholangitis include an indwelling biliary stent, malignant biliary obstruction, previous hospitalization and antibiotic use within 90 days.

\section{What is the Empirical Antibiotic Regimen for Acute Cholangitis?}

\section{Evidence Statement}

The empirical antibiotic regime in patients with acute cholangitis is guided by the severity of the disease, local antibiotic susceptibility pattern and biliary penetration of the antibiotics. Duration of antibiotics depends on the severity of cholangitis and adequacy of source control. Biliary drainage (percutaneous or endoscopic) is required in addition to antibiotic use in the management of acute cholangitis.

\section{Recommendations}

- Empirical antibiotic therapy should be guided by the severity of the cholangitis, local microbiological susceptibility patterns, biliary penetration of antibiotics and previous antibiotic exposure (UPP).

- We recommend either beta-lactam/beta-lactamase inhibitor (such as cefoperazone-sulbactam or piperacillin/tazobactam) or carbapenems (imipenem/ meropenem) as monotherapy in patients with moderate to severe cholangitis (3B).

- We recommend antibiotic duration for 4-7 days in patients of acute cholangitis after adequate source control (2B).

- Biliary drainage should be considered in all patients with cholangitis in addition to empirical antibiotic therapy (1A).

\section{LIVER ABSCESS}

\section{Incidence and Risk Factors}

\section{What are the most common organisms causing a liver abscess in ICU?}

\section{Evidence Statement}

Amoebic liver abscess is the most common cause of liver abscess in Indian setup. The incidence of pyogenic liver abscess varies from 2.3 to 446 per 100000 hospital admissions per year. Gram-negative organisms (E. coli and Klebsiella) are the most common organisms causing a pyogenic liver abscess. Risk factors for pyogenic liver abscess include diabetes mellitus, older age, male gender, biliary diseases, biliary procedures, alcoholism, malignancy, intraabdominal infection, and cystic lesions in the liver.

\section{What are the Empirical Antibiotics of Choice for Treating a liver Abscess in ICU?}

\section{Amoebic liver abscess}

\section{Evidence Statement}

Metronidazole is the drug of choice for treatment of amoebic liver abscess. The optimum duration of treatment in patients with an amoebic liver abscess is 10-14 days. Routine needle aspiration of amoebic liver abscess is controversial. Addition of aspiration to drug therapy in patients with amoebic liver abscess of $>5 \mathrm{~cm}$ in size hastens clinical improvement.

\section{Recommendations}

- We recommend metronidazole as an initial antibiotic of choice in patients with an amoebic liver abscess (2A).

- We recommend antibiotic treatment for 10-14 days in patients with an amoebic liver abscess (3B).

- Needle aspiration of amoebic liver abscess is recommended in patients with lack of clinical improvement in 48 to 72 hours, left lobe abscess, abscess more than 5 to $10 \mathrm{~cm}$ or thin rim of liver tissue around the abscess $(<10 \mathrm{~mm})$ (UPP).

\section{Pyogenic Liver Abscess}

\section{Evidence Statement}

Beta-lactam/beta-lactamase inhibitors, metronidazole, and carbapenems are effective antibiotics for the management of pyogenic liver abscess. Carbapenems are effective in case of suspected infection with ESBL producing organisms or melioidosis. Antibiotics are required for prolonged periods ranging from 2 to 4 weeks. Clinical and radiological assessment is required to guide the adequate treatment duration.

\section{Recommendations}

- We recommend beta-lactam/beta-lactamase inhibitors with metronidazole in patients with pyogenic liver abscess for a duration of 2 to 4 weeks (2A).

- We recommend carbapenems in case of infection with ESBL producing organisms or melioidosis (2B).

\section{PERITONITIS}

\section{What are the Most Common Organisms Causing Peritonitis in ICU?}

\section{Evidence Statement}

The risk factors for the development of primary peritonitis are decompensated cirrhosis, nephrotic syndrome and peritoneal dialysis. The risk factors for the development 
of secondary peritonitis include intra-abdominal organ perforation, post-intra-abdominal surgery, and trauma. Longer ICU stay, urgent operation on hospital admission, total parenteral nutrition, and stomachduodenum as primary infection site are associated with the development of tertiary peritonitis. Gram-negative enteric organisms are the common causes of primary and secondary peritonitis. Other organisms include grampositive as well as anaerobic bacteria. The organisms commonly isolated in tertiary peritonitis are Candida, Enterococcus faecium and Staphylococcus epidermidis.

\section{What are the Empirical Antibiotics of Choice for Treating Peritonitis in ICU?}

\section{Evidence Statement}

Third generation cephalosporins are the most effective antibiotic therapy for primary peritonitis. Antibiotics are usually required for 7 to 10 days for adequate treatment. Most of the organisms isolated in secondary peritonitis are sensitive to beta-lactam/beta-lactamase inhibitors or carbapenems. For gram-positive organisms, vancomycin and linezolid are effective treatment options. The short duration of antibiotic treatment (4 days) is as effective as longer duration after an adequate source control.

\section{Recommendations}

- We recommend third-generation cephalosporins (such as cefotaxime and ceftriaxone) for 7 to 10 days in patients with primary peritonitis (2A).

- We recommend either the beta-lactam/beta-lactamase inhibitor or carbapenems with an anaerobic cover (using metronidazole) for the treatment of secondary peritonitis (2A).

- For secondary peritonitis antibiotic treatment is required for 4 days after an adequate source control (2A).

\section{CNS INFECTIONS IN ICU}

\section{What are the Most Common Organisms Causing Acute Bacterial Meningitis in ICU?}

\section{Community-acquired Meningitis}

\section{Evidence Statement}

The incidence of community-acquired pyogenic meningitis ranges from 2 to 7.40 per lakh population. The common causative organisms include Streptococcus pneumoniae, Neisseria meningitides, other streptococci, Haemophilus influenzae, and Listeria monocytogenes. Other causative organisms are Staphylococcus species, gramnegative bacilli, Pseudomonas, and Acinetobacter. Common risk factors for community-acquired bacterial meningitis are otitis media, elderly population, depressed immune status and prior use of antibiotics.

\section{Nosocomial Meningitis}

\section{Evidence Statement}

The incidence of post ventricular drain or catheter meningitis ranges from 2 to $27 \%$. Commonly implicated organisms are CONS (especially Staphylococcus epidermidis), Staphylococcus aureus, Acinetobacter, Pseudomonas, and Enterobacteriaceae. Risk factors are repeated catheterization, higher catheter duration, CSF sampling, the presence of concomitant systemic infection and surgical technique, i.e., subcutaneously tunneled extraventricular drain (EVD), Rickham reservoir with percutaneous CSF drainage. The incidence of post craniotomy or post neurosurgery meningitis is $0.02 \%$ to $9.5 \%$. Most commonly implicated organisms are Staphylococcus aureus, coagulase-negative staphylococci (especially S. epidermidis), Enterobacteriaceae, Acinetobacter and Pseudomonas. Risk factors include CSF leak, EVD, longer duration of drainage, multiple operations, lack of antibiotic prophylaxis and emergency surgery. The incidence of post-neuroaxial blockade meningitis is 0.2 per 10000 with viridans streptococci and Staphylococcus aureus being common organisms. Exogenous inoculation is the main risk factor. Post head trauma meningitis incidence ranges from $1.39 \%$ to $2 \%$ with CONS, and Enterobacteriaceae as common microbes and prolonged hospitalization, insertion of the lumbar and ventricular drain as common risk factors. Post internal ventricular drain infection incidence ranges from 5.9 to $15.2 \%$. Most common causative organisms are CONS, Staphylococcus aureus, gram-negative bacilli, group D streptococci, and Propionibacterium acnes. CSF leak, single gloves use and a number of times shunt exposed to breached surgical gloves are the risk factors.

\section{What are the Empirical Antibiotics of Choice for Treating Acute Bacterial Meningitis in ICU? What Should be the Duration of Antibiotic Treatment?}

\section{Community-acquired Meningitis}

\section{Evidence Statement}

Choice of antibiotics depends on the most likely causative micro-organism, local antibiotics sensitivity patterns, mechanism of infection and patient's predisposing condition. Most commonly recommended empirical antibiotic regimens include third-generation cephalosporin plus vancomycin, third-generation cephalosporin monotherapy and penicillin monotherapy. Addition of amoxicillin, ampicillin or benzyl-penicillin has been recommended in patients older than 50 years. 


\section{Recommendations}

- We recommend third-generation cephalosporin (preferably ceftriaxone) plus vancomycin as empirical antibiotics of choice for community-acquired meningitis (3A).

- We recommend adding ampicillin or amoxicillin if age $>50$ years. $(3 \mathrm{~A})$.

- If beta-lactams are contraindicated, we recommend chloramphenicol plus vancomycin as antibiotic of choice, and to add cotrimoxazole, if age $>50$ years (3A).

- We recommend ciprofloxacin or aztreonam plus vancomycin as alternative regimen and to add cotrimoxazole if age greater than 50 years (UPP).

- We recommend a duration of antibiotics based on suspected or isolated organisms, i.e., 10 to 14 days for Streptococcus pneumoniae, 14 to 21 days for Streptococcus agalactiae, 7 days for Neisseria meningitides or Haemophilus influenzae, 21 days for aerobic gram-negative bacilli, and 21 days or more for Listeria monocytogenes (3A).

- If no microorganism is identified, treatment should be given for at least 10 to 14 days (3A).

\section{Nosocomial Meningitis}

\section{Evidence Statement}

Vancomycin in combination with cefepime, ceftazidime or meropenem is commonly recommended an empirical antibiotic regimen for nosocomial meningitis. Alternative regimens include third-generation cephalosporin or meropenem monotherapy or ceftriaxone plus flucloxacillin or cloxacillin combination therapy. Limited available evidence shows the efficacy of intraventricular or intrathecal antibiotics in the management of nosocomial meningitis poorly responsive to systemic antibiotics.

\section{Recommendations}

- We recommend vancomycin plus cefepime or ceftazidime or meropenem as empirical antibiotics of choice for nosocomial meningitis (3A).

- Colistin may be given if the incidence of CRE or drugresistant Acinetobacter is high in the specific unit (UPP).

- If beta-lactams are contraindicated, we recommend replacing b-lactam with aztreonam or ciprofloxacin (3A).

- Intraventricular/intrathecal antibiotics should be considered if infection responds poorly to appropriate systemic antibiotics clinically or microbiologically (3A).

\section{What are the Most Common Organisms Causing Brain Abscess in ICU?}

\section{Evidence Statement}

The incidence of brain abscess ranges from 1.3 to 2.6 cases per lakh population. Most commonly involved micro- organisms include Streptococcus (especially S. viridans), Staphylococcus (especially S. aureus), gram-negative bacilli, anaerobes (Bacteroides, PeptoStreptococcus, Fusobacterium), Pseudomonas and $H$. influenzae. Polymicrobial etiology accounts for 23 to $26 \%$ cases. Risk factors include otitis media, sinusitis, head trauma, congenital heart diseases, hematogenous spread, surgery, immunocompromised status, pulmonary disease, meningitis, and odontogenic infections.

\section{What are the Empirical Antibiotics of Choice for Treating Brain Abscess in ICU? What Should be the Duration of Antibiotic Treatment?}

\section{Evidence Statement}

The most common empiric treatment consists of a thirdgeneration cephalosporin combined with metronidazole. Antibiotic duration ranges from 4 to 8 weeks.

\section{Recommendations}

- We recommend 3rd generation cephalosporins plus metronidazole as the empirical antibiotic of choice for brain abscess (3A).

- We recommend adding vancomycin if a high likelihood of MRSA (3A).

- We recommend vancomycin plus ciprofloxacin if beta-lactams are contraindicated (3A).

- We recommend aztreonam if ciprofloxacin cannot be given or contraindicated (UPP).

- We recommend a minimum 4 weeks of therapy; however, duration may be extended according to clinic-radiological response irrespective of aspiration or excision of the abscess (3A).

\section{SKIN AND SOFT TISSUE INFECTIONS (SSTI) IN ICU}

\section{What are the Most Common Organisms and Risk Factors for SSTI in ICU?}

\section{Evidence Statement}

Older age, diabetes mellitus, obesity, malignancy, cirrhosis, and longer ICU stay are risk factors for SSTIs. Gram-positive organisms (Staphylococcus aureus) are the most common organism responsible for the SSTIs. E. coli and Pseudomonas are common pathogens among gram-negative organisms. MRSA and ESBL producing gram-negative organisms are the most common causative agents for SSTIs in ICU. Monomicrobial necrotizing fasciitis is commonly caused by Streptococcus pyogenes; mixed coliforms, anaerobes, and staphylococci are common causes of polymicrobial necrotizing fasciitis. 


\section{What are the Empirical Antibiotics of Choice for Treating SSTI in ICU? For Empirical Therapy, Should Combination Therapy be Preferred Over Monotherapy?}

\section{Evidence Statement}

Vancomycin, teicoplanin, daptomycin, and linezolid are effective in SSTIs caused by MRSA. Piperacillin-tazobactam and carbapenems are the most effective antibiotics for ESBL producing gram-negative organisms. Penicillin plus clindamycin are most effective antibiotics in monomicrobial necrotizing fasciitis, whereas a combination of piperacillin-tazobactam, fluoroquinolone and clindamycin are effective for polymicrobial necrotizing fasciitis.

\section{Recommendations}

- For moderate non-purulent SSTI, we recommend intravenous penicillin or clindamycin as the first choice of antibiotics (2A).

- Severe non-purulent SSTI should be treated with a combination of piperacillin-tazobactam along with coverage for MRSA (vancomycin, teicoplanin, daptomycin or linezolid) (2A).

- Concomitant surgical inspection or debridement should be considered for severe non-purulent SSTIs (2A).

- For severe purulent SSTI, incision and drainage followed by empiric antibiotics including piperacillintazobactam, along with MRSA coverage (vancomycin, teicoplanin, daptomycin or linezolid) is recommended (3A).

- Penicillin plus clindamycin is recommended for monomicrobial necrotizing infection caused by Streptococcus pyogenes or clostridial species. For polymicrobial necrotizing fasciitis, a combination of piperacillin-tazobactam, fluoroquinolone, and clindamycin is recommended (3A).

\section{What Should be the Duration of Antibiotic Treatment for SSTI?}

\section{Evidence Statement}

A shorter course of antibiotic therapy is adequate for uncomplicated SSTIs while complicated SSTIs require a longer duration of antibiotic therapy.

\section{Recommendations}

- Severe nonpurulent SSTIs should be treated with at least 5 days of antibiotics (3A).

- Severe SSTIs with organ dysfunction should be treated with a prolonged course of antibiotics of 2-3 weeks duration (3A).

\section{SEPSIS OF UNKNOWN CAUSE IN ICU}

\section{What is the Empirical Treatment for Sepsis of Unknown Cause in ICU?}

\section{Evidence Statement}

Empirical therapy with dual class (with different mechanisms of action) combination antimicrobial therapy for sepsis of unknown cause in ICU is associated with have better clinical outcomes. Empirical therapy with either piperacillin-tazobactam or carbapenems in combination with aminoglycoside or fluoroquinolone has been shown to give appropriate broad coverage leading to better clinical outcomes as compared to monotherapy.

\section{Recommendations}

- We recommend empirical antimicrobial therapy with a combination of ceftriaxone and doxycycline or macrolide for community-acquired sepsis of unknown origin in ICU (UPP).

- We recommend empirical antimicrobial therapy with a combination of beta-lactam/beta-lactamase inhibitor and a fluoroquinolone or aminoglycoside for nosocomial sepsis of unknown origin in ICU (UPP).

- Empiric therapy should attempt to provide antimicrobial activity against the most likely pathogens based upon clinical features along with local patterns of infection and resistance (UPP).

- Duration of therapy is 7 to 10 days, though longer courses may be appropriate in patients with slow response (3B).

\section{EMPIRICAL ANTIFUNGALS FOR NON-NEUTROPENIC PATIENTS IN ICU}

\section{What are the Risk Factors for Invasive Fungal Infections in ICU?}

\section{Evidence Statement}

Risk factors for invasive fungal infections in non-neutropenic patients in ICU are surgery, total parenteral nutrition, renal replacement therapy, cardiopulmonary bypass $>120$ minutes, diabetes mellitus, central venous catheters, urinary catheters, Candida colonisation with colonization index $>$ 0.5 , use of broad-spectrum antibiotics, acute renal failure, mechanical ventilation $>3$ days and APACHE II score $>16$.

\section{What is the Role of Empirical Antifungals in Non-neutropenic Patients in ICU?}

\section{Evidence Statement}

Empirical antifungals for non-neutropenic patients in ICU routinely has not been associated with a decrease in mortality or hospital length of stay. Empirical antifungals in 
patients at high risk for invasive fungal infections in ICU has been shown to reduce the incidence of subsequent proven invasive fungal infections.

\section{Recommendations}

- We do not recommend the routine use of empirical antifungals in non-neutropenic patients in ICU (1A)

- Empirical antifungals may be considered in critically ill patients with a high risk of invasive fungal infections to reduce the incidence of subsequent invasive fungal infections (1B).

\section{What is the Antifungal Agent of Choice and Duration of Empirical Therapy in Non-neutropenic Patients in ICU?}

\section{Evidence Statement}

Fluconazole and caspofungin are useful as empirical antifungal therapy in non-neutropenic ICU patients at high risk of Invasive fungal infection. In India, the rate of fluconazole resistance is up to $7 \%$, especially in nonalbicans Candida species.

\section{Recommendations}

- We recommend fluconazole or caspofungin as preferred empirical antifungal agents in non-neutropenic ICU patients at risk for invasive fungal infection (1A).

- Caspofungin may be preferred in areas with high prevalence of fluconazole resistance (1B).

- Micafungin or anidulafungin may be used as alternative agents (3A).

- Recommended duration of empirical antifungal therapy is 2 weeks. (3A)

\section{Antibiotic Stewardship}

\section{Evidence Statement}

Antibiotic stewardship programs in hospitalized patients are associated with a reduction in a number of antibiotic days, duration of hospital stay and all-cause mortality.

\section{Recommendations}

- All hospitals should have an antibiotic stewardship program including the intensive care units (2A).

\section{What are the Essential Strategies of Antibiotic Stewardship in an ICU Setting?}

\section{Evidence Statement}

Antibiotic stewardship requires a multidisciplinary approach with integration of infectious disease physician, a microbiologist with logistic and financial support from hospital administration. Both enablement and restrictive strategies are useful in improving adherence to antibiotic stewardship programs. Restrictive strategies give immediate results. Enablement practices are more resource intensive. Most studies have used a combination of both the methods and have shown additive effects. Providing feedback to the treating team improves adherence. Asingle RCT has shown that a restrictive strategy alone may cause a delay in the initiation of antibiotics.

\section{Recommendations}

- Prospective audit of antibiotic use and / or preauthorization (if feasible) along with feedback to the treating team is recommended as part of an antibiotic stewardship program (1A).

\section{What is the Role of Antibiotic Cycling, Intravenous to Oral switch and De-escalation in the ICU?}

\section{Evidence Statement}

Antibiotic cycling in the intensive care unit has not been adequately studied in randomized controlled trials. Nonrandomized studies show significant heterogeneity in terms of site of study, a method of cycling and confounders like simultaneous infection control measures being employed. Evidence of benefit of antibiotic cycling is lacking, with few studies demonstrating a reduction in colonization though mortality and length of hospital stay remain unchanged.

\section{Recommendations}

- Antibiotic cycling should not be used as a method of antibiotic stewardship program (2A).

\section{Scheduled Intravenous to Oral Switch}

\section{Evidence Statement}

Early intravenous to the oral transition of antibiotics reduce hospital length of stay and cost of care. There is no increase in mortality or other adverse events when this is done after assessing which patients can be safely transitioned to oral therapy.

\section{Recommendations}

- Antibiotic stewardship programs should implement strategies to improve the timely transition from parenteral to oral antibiotic therapy (2A).

\section{De-escalation in Intensive Care Unit}

\section{Evidence Statement}

Pooled results from observational studies in an ICU setting do not show any increase in mortality with antibiotic de-escalation while significantly reducing antibiotic exposure days and ICU length of stay. 


\section{Recommendations}

- Antibiotic de-escalation in the ICU is recommended as part of an antibiotic stewardship program (2A).

\section{What is the Role of Procalcitonin in Antibiotic De-escalation ICU?}

\section{Evidence Statement}

Implementation of antibiotic de-escalation algorithm based on serial procalcitonin measurements has been shown to reduce mortality, length of ICU stay, the total duration of antibiotic days and health care costs.

\section{Recommendations}

- Procalcitonin based algorithms may be used for antibiotic de-escalation (1A).

\section{INTRODUCTION}

Severe infections are among the common indications requiring admission to intensive care units (ICU). All physicians, irrespective of the specialty, deal with such patients. For these patients, effective antibiotic therapy is life-saving. The resistance to currently available antibiotics is increasing over the last few years. Secondly, only a few new antibiotics have been marketed during the last few years and will be made available in the coming years. Therefore, the best way to preserve the efficacy of existing antibiotics remains the appropriate use of these drugs. One way to do this may be to increase awareness and develop guidelines for the prescription of antibiotics. There are international as well as Indian guidelines covering some of the common infections encountered in ICU. However, none of the existing guidelines have comprehensively addressed the issue of empirical antibiotic prescription in ICU as a whole. Therefore, these guidelines are the consensus of experts from all over the country based upon available scientific literature.

\section{Scope of Guidelines}

The scope of these guidelines includes an antibiotic prescription for common bacterial infections for pneumonia (community acquired, hospital-acquired and ventilatorassociated), bloodstream infections, abdominal infection (hepato-biliary, pancreatic, urogenital), central nervous system, skin and soft tissue infections in patients admitted in ICU. These guidelines are for immunocompetent patients. The antibiotic prescription for critically ill immunocompromised patients is dealt in part II of this supplement.

\section{METHODOLOGY}

The guidelines for antibiotic prescription in intensive care unit were framed by the Department of Pulmonary
Medicine and Sleep Disorders, All India Institute of Medical Sciences, New Delhi under the aegis of Indian Society of Critical Care Medicine. The committee included experts (list enclosed) from various realms dealing with ICU infections, i.e., Critical Care, Pulmonary Medicine, Gastroenterology, Neurology, Nephrology, and Microbiology. The experts were divided into five groups. Review of literature was performed by searching various electronic databases including Pubmed and Embase. Cross-references from articles and all major international guidelines on the topics were also reviewed.

The experts in each group exchanged and reviewed relevant literature, and the consensus was derived on the scope and questions that needed to be answered in the formulation of the guidelines. The final expert committee meeting was held over two days at the All India Institute of Medical Sciences, New Delhi. After detailed discussion, guidelines were framed after a thorough review of the literature.

Modified grade system was utilized to classify the quality of evidence and the strength of recommendations (Table 1). The draft document thus formulated was reviewed by all committee members; comments and suggestions were incorporated after discussion, and a final document was prepared. The final document was reviewed and accepted by all expert committee members.

\begin{tabular}{|c|c|}
\hline \multicolumn{2}{|c|}{$\begin{array}{l}\text { Table 1. Criteria for level of evidence and grading of } \\
\text { strength of recommendations used in } \\
\text { formulation of current guidelines }\end{array}$} \\
\hline Quality of Evidence & Level \\
\hline $\begin{array}{l}\text { Evidence from } \geq 1 \text { good quality and well } \\
\text { conducted randomized control trial(s) or meta- } \\
\text { analysis of RCT's }\end{array}$ & 1 \\
\hline $\begin{array}{l}\text { Evidence from at least } 1 \mathrm{RCT} \text { of moderate } \\
\text { quality, or well-designed clinical trial without } \\
\text { randomization; or from cohort or case-controlled } \\
\text { studies. }\end{array}$ & 2 \\
\hline $\begin{array}{l}\text { Evidence from descriptive studies, or reports } \\
\text { of expert committees, or opinion of respected } \\
\text { authorities based on clinical experience }\end{array}$ & 3 \\
\hline $\begin{array}{l}\text { Not backed by sufficient evidence; however, a } \\
\text { consensus reached by the working group, based } \\
\text { on clinical experience and expertise }\end{array}$ & $\begin{array}{l}\text { Useful } \\
\text { Practice } \\
\text { Point } \\
\text { (UPP) }\end{array}$ \\
\hline Strength of Recommendations & Grade \\
\hline $\begin{array}{l}\text { Strong Recommendations to do (or not to do) } \\
\text { where the benefits clearly outweigh the risk (or } \\
\text { vice versa) for most, if not all patients }\end{array}$ & A \\
\hline $\begin{array}{l}\text { Weak Recommendations, where benefits and } \\
\text { risk are more closely balanced or are more } \\
\text { uncertain }\end{array}$ & B \\
\hline
\end{tabular}

\section{Pharmacokinetics and Pharmacodynamics}

Pharmacokinetics deals with the time course of drug absorption, distribution, metabolism, and excretion while pharmacodynamics involves the relationship between 
drug concentration and its effects including toxicity. Each antibiotic has its pharmacokinetic profile through each class of antibiotics has its class-specific properties as well. Each class of antimicrobials has a different pharmacodynamic profile based on different inhibitory characteristics on bacteria.

Individualized dosing regimens using known pharmacokinetics and pharmacodynamic characteristics are important to optimize patient outcomes and minimize antimicrobial resistance. Pharmacokinetic profiles change over time in critically ill patients, warranting periodic reconsideration of dosing regimens.

The factors determining metabolism and effects of an antibiotic include basic antibiotic characteristics such as lipophilic or hydrophilic, patient statuses such as volume status and end organ function and changes in pathophysiologic characteristics, i.e., systemic inflammation and hemodynamics. Hydrophilic antibiotics have a low volume of distribution, predominantly renal clearance and low intracellular penetration as compared to lipophilic antibiotics. Examples of hydrophilic antibiotics include beta-lactams, aminoglycosides, vancomycin, linezolid, and colistin while lipophilic antibiotics are fluoroquinolones, macrolides, clindamycin and tigecycline. ${ }^{1}$

The antibiotics can be broadly classified into those with concentration-dependent killing activity and those with time-dependent killing activity. The examples of former include aminoglycosides, fluoroquinolones, metronidazole, colistin, and clindamycin whereas that of latter include beta-lactams, linezolid, and tetracyclines.

Sepsis affects drug metabolism by various mechanisms. Being a hyperdynamic state it (pharmacologically or pathophysiologically enhanced) can increase creatinine clearance and hepatic perfusion thus increasing drug removal. At the same time, sepsis-induced organ-dysfunction can reduce metabolism and elimination of the active drug. Renal replacement therapies can increase clearance for some drugs like piperacillintazobactam and meropenem. The body has adaptive methods for increasing drug clearance during states of multiorgan failure. For example, gastrointestinal clearance of ciprofloxacin is increased in renal failure while biliary clearance of piperacillin increases in renal failure. Serum protein concentration also affects the antibiotic concentration. Significant changes in free fractions of the drug are only relevant for highly protein-bound drugs (>95\%). Small changes in protein binding result in huge relative changes in free (unbound) drug. Changes in protein binding will affect both clearances as well as the volume of distribution. Most antibiotics have low protein binding (<90\%) except ceftriaxone ( $95 \%$ bound to albumin), ertapenem, teicoplanin, aztreonam, and daptomycin.
An open-label RCT involving 140 patients with sepsis compared continuous infusion of beta-lactams with intermittent infusion and demonstrated higher clinical cure rates and higher ventilator-free days in continuous infusion group without any mortality difference between the two groups. ${ }^{2}$ Similar results have been found in other studies as well through a double-blind study by Dulhunty et al did not find any difference in ICU-free days, 90-day survival and clinical cure between continuous infusion and intermittent infusion groups. ${ }^{3}$ An individual patient data meta-analysis found significantly lower hospital mortality rates with continuous infusion of beta-lactams as compared to intermittent infusion in patients with severe sepsis. ${ }^{4}$ Regarding vancomycin, a meta-analysis including 11 studies comparing continuous versus intermittent infusion found that patients treated with continuous infusion had a significantly lower incidence of nephrotoxicity without any difference in treatment failure and mortality. ${ }^{5}$

\section{Evidence Statement}

Time-dependent antibiotics require drug concentrations greater than the minimum inhibitory concentration (MIC) for a certain time period between doses, which usually ranges from 40 to $50 \%$ of the inter-dose interval for their best action. Continuous infusions are preferred overextended infusions for beta-lactam antibiotics and are associated with clinical benefits like a decrease in hospital stay, cost of therapy and mortality. For vancomycin, continuous infusion is associated with reduced toxicity and cost of therapy without any mortality benefit.

\section{Community-Acquired Pneumonia in the Intensive Care Unit}

Community-acquired pneumonia (CAP) refers to symptoms suggestive of acute lower respiratory tract illness (a cough with or without expectoration, dyspnea, pleuritic chest pain) along with systemic manifestations (fever, chills, rigors or severe malaise), clinicoradiologic evidence (like crepitations or bronchial breath sounds;lobar or patchy consolidation or interstitial infiltrates) and no other explanation for the illness. ${ }^{6,7}$ CAP can simply be defined as pneumonia which is not acquired in hospital or long-term care facility. ${ }^{8}$

\section{What are the Common Organisms Causing Community-acquired Pneumonia in Intensive Care Unit Worldwide and India?}

Common organisms causing CAP requiring intensive care admission worldwide include Streptococcus pneumoniae (12-43\%), Hemophilus influenza (0-12\%), Legionella pneumophila (0-30\%), Staphylococcus aureus (0-19\%), 
gram-negative enteric bacilli (0-27\%), Mycoplasma pneumoniae $(0-7 \%)$, Chlamydia species $(0-2 \%)$, Coxiella burnetti (0-2\%), and viruses (0-17\%) including influenza (0-9\%). ${ }^{9}$ In a recent active population-based surveillance study, Streptococcus pneumoniae, Staphylococcus aureus, and Enterobacteriaceae were more commonly implicated in CAP requiring intensive care $(\mathrm{p}<0.001) .{ }^{10}$ Methicillin resistant Staphylococcus aureus (MRSA) remains an infrequent but important cause of CAP in intensive care unit (ICU) settings; however, evidence regarding prevalence and risk factors is limited to few observational studies, case series and case reports. ${ }^{11-14}$

The literature on the epidemiology of CAP in India comes from hospital-based observational studies and surveillance data as the ICU specific studies are not available. Streptococcus pneumoniae (2-35.8\%), Mycoplasma pneumoniae (3-24\%), Chlamydia pneumoniae (6-18\%), Legionella spp. (2-15\%), Mycobacterium tuberculosis (0-5\%), Haemophilus influenzae (0-15.4\%), Staphylococcus aureus (2-13\%), Klebsiella pneumoniae (3-25.5\%), other gram negative bacilli (0-19\%) are the common organisms implicated in CAP requiring hospitalization in India. ${ }^{15-}$ ${ }^{38}$ High prevalence of Staphylococcus aureus (26.7\%) and MRSA causing CAP (60.9\% of staphylococci) has been reported in one Indian study. ${ }^{16}$

Increasing age, active smoking, chronic obstructive pulmonary disease (COPD) and diabetes mellitus appear to be significant risk factors for the development of severe CAP. Structural lung disease and COPD are risk factors for infection due to Pseudomonas aeruginosa. ${ }^{6,39,40}$

Streptococcus pneumoniae largely remains sensitive to amoxicillin-clavulanic acid and azithromycin with only a few studies reporting resistance to amoxicillin-clavulanic acid $(20 \%)$, levofloxacin $(20 \%)$ and azithromycin $(13 \%) .{ }^{6,24,25,35}$ There is limited data on antibiotic sensitivity patterns of other microbes. $H$. influenzae also seems to be largely sensitive to amoxicillin clavulanic acid and azithromycin; in one study, 23\% isolates were resistant to amoxicillin-clavulanic acid, 13\% were resistant to azithromycin whereas only $6 \%$ were resistant to cefuroxime. ${ }^{35}$ Gram negative bacilli (GNB) are usually sensitive to beta-lactams and fluoroquinolones. ${ }^{33}$ However, in recent studies, prevalence of extended spectrum $\beta$-lactamase (ESBL)organisms appears to be increasing with resistance to carbapenems (16.6\%), piperacillin-tazobactam (39.5\%), and cefoperazonesulbactam $(42 \%)$ reported in a recent prospective study. ${ }^{35}$

\section{Evidence Statement}

Streptococcus pneumoniae, gram-negative bacilli (including Klebsiella, Hemophilus influenzae), atypical organisms (Mycoplasma pneumoniae) and viruses (including influenza) are common causes of community-acquired pneumonia (CAP) in intensive care unit (ICU). Staphylococcus aureus, Legionella, and Mycobacterium tuberculosis are less common causes of CAP in ICU. Pseudomonas aeruginosa is an important pathogen causing CAP in patients with structural lung disease. Methicillin-resistant Staphylococcus aureus (MRSA) and multidrug-resistant gram-negative organisms are relatively infrequent causes of CAP in India and are associated with risk factors such as structural lung disease and previous antimicrobial intake. Anaerobic organisms may cause CAP or co-infection in patients with risk factors for aspiration like elderly, altered sensorium, dysphagia, head, and neck malignancy. S. pneumoniae remains sensitive to betalactams and macrolides. Hemophilus influenzae has good sensitivity to beta-lactam with beta-lactamase inhibitors and fluoroquinolones. Recent studies show an increasing prevalence of extended spectrum $\beta$-lactamase (ESBL) producing Enterobacteriaceae.

\section{What are the Risk Factors for Multidrug-resistant (MDR) Pathogens for CAP in ICU?}

Age more than 65 years, chronic respiratory disease, and prior antibiotic treatment were associated with increased risk of CAP due to multidrug-resistant (MDR) pathogens in prospective observational studies. ${ }^{41-44}$ Other factors associated with increased risk of MDR CAP include prior hospitalization for more than 48 hours in the last 3 months, home infusion therapy and patients on renal replacement therapy. Immunosuppression was also considered to be a risk factor for CAP due to MDR organisms. ${ }^{6}$

\section{Evidence Statement}

Risk factors for multidrug-resistant (MDR) organisms include age $>65$ years, antimicrobial therapy in the preceding 3 months, high frequency of antibiotic resistance in the community, hospitalization for $\geq 48$ hours in the preceding 3 months, home infusion therapy including antibiotics, home wound care, chronic dialysis within 1 month, family member with MDR pathogen and ongoing immunosuppressive treatment.

\section{Recommendations}

- All patients admitted with CAP in ICU should be evaluated for risk factors for infection with MDR organisms (2A).

- Antibiotic therapy should be individualized to cover the commonly implicated organisms according to risk factors, including Pseudomonas, ESBL producing Enterobacteriaceae or MRSA (3A). 


\section{How Early Should the Antibiotics be Initiated in patients with CAP Who Require ICU Admission?}

In retrospective studies on $\mathrm{CAP}$, initiation of antibiotics within 4 hours of presentation has been associated with a reduction in all-cause mortality, regardless of severity [relative risk (RR) $0.24 ; 95 \%$ confidence interval (CI) 0.08-0.71]. ${ }^{45}$ A systematic review of prospective studies also favored early administration of antibiotics; however, the confidence interval was wide (RR 0.82; 95\% CI 0.54-1.24). ${ }^{45}$ A recent meta-analysis of retrospective studies also showed decreased all-cause mortality with early administration of antibiotics before 4 hours of hospital admission, especially in severe CAP with pneumonia severity index (PSI) IV to $\mathrm{V}$ (adjusted odds ratio, AOR $0.87 ; 95 \%$ CI 78-97). However, no significant benefit was shown in clinical stability at 48 hours (AOR 1.04; 95\% CI 0.75-1.44), length of hospital stay (AOR 0.92; 95\% CI $84-1.01 \%$ ) or readmission after discharge (AOR 0.99; 95\% CI 0.88-1.11\%). ${ }^{8}$ However, all the included studies were retrospective or chart reviews, with low quality of evidence. There was no significant mortality benefit with the administration of antibiotics before one hour of recognition of severe sepsis or septic shock (pooled odds ratio $1.46,95 \%$ CI $0.89-2.4$ ) in a recent meta-analysis. Out of 18 eligible studies, 7 studies were excluded due to non-availability of data confounding the findings. ${ }^{46}$ In a recent retrospective study of 35,000 randomly selected in patients with sepsis, each hour delay in administration of antibiotics was associated with increased odds of in-hospital mortality in patients with sepsis (Odds ratio, OR 1.09 ; 95\% CI 1.00-1.19; $\mathrm{p}=0.046)$, severe sepsis (OR $1.07 ; 95 \%$ CI 1.01-1.24; $\mathrm{p}=0.014)$ and septic shock (OR $1.14 ; 95 \%$ CI $1.06-1.23 ; \mathrm{p}=0.001) .{ }^{47}$

\section{Evidence Statement}

Early initiation of antibiotics has been associated with a reduction in all-cause mortality in community-acquired pneumonia, including severe pneumonia with sepsis or septic shock.

\section{Recommendations}

- Appropriate antimicrobial therapy should be initiated as early as possible in patients of CAP requiring ICU admission, preferably within the first hour after obtaining necessary microbiologic samples (3A).

\section{Should CAP in ICU Receive Empirical Antimicrobials or Upfront Targeted Antimicrobial Therapy?}

Targeted antibiotic therapy based on Legionella and pneumococcal urinary antigen testing was associated with higher relapse rate without any significant differences in clinical failure, length of hospital stay or clinical failure in a randomized controlled trial in patients with severe CAP. However, the study was inadequately powered for outcomes as less than $50 \%$ of patients had PSI IV, and V CAP and only one patient required ICU admission. ${ }^{48}$ In another randomized controlled trial, targeted antibiotic therapy based on respiratory secretions cultures, blood cultures, paired serum samples (for Mycoplasma, Chlamydia, and Coxiella) and urinary antigens (for Pneumococcus and Legionella) was similar to empirical therapy in terms of clinical cure, length of hospital stay and late treatment failure or relapse. The study was inadequately powered for ICU patients, though it demonstrated significantly reduced mortality $(45 \%$ vs. $91 \%$; $\mathrm{p}=0.02)$ with targeted therapy as compared to empirical therapy. ${ }^{49}$ Similarly, in a large retrospective study, targeted antibiotic therapy has been associated with reduced 30-day mortality (AOR 0.64, 95\% CI 0.56-0.74) in CAP, severe CAP (AOR 0.70; 95\% CI 0.54-0.91)and very severe CAP (AOR 0.51, 95\% CI 0.40 to 0.64$).{ }^{8,50}$ Other retrospective studies have demonstrated the limited utility of diagnostic testing to influence prescription modification, clinical cure or failure though lower mortality is reported with targeted therapy (RR 0.37, 0.24-0.57). ${ }^{8,51}$ Obtaining blood cultures before initiating therapy was associated with a mortality benefit in a large retrospective study in 14069 patients with CAP requiring hospitalisation. ${ }^{52}$

\section{Evidence Statement}

Early institution of targeted antibiotic therapy in severe CAP based on urinary antigen testing is associated with higher relapse rate without any mortality benefit in prospective randomized studies. Retrospective studies have shown mortality benefit with narrowing down of antibiotic therapy based on results from cultures of respiratory specimens, blood cultures as well as Legionella and pneumococcal urinary antigen testing.

\section{Recommendations}

- Empirical therapy covering common etiologic organisms should be initiated for severe CAP requiring ICU admission (2A).

- Investigations including the culture of respiratory secretions (sputum, endotracheal aspirate), blood cultures, urinary antigen testing for Pneumococcus and Legionella may be performed to narrow down therapy. Bronchoscopic BAL or protected specimen brush samples or polymerase chain reaction (PCR) for viral etiology may be performed for microbiologic diagnosis on a case by case basis (3A). 


\section{For Empirical Therapy in Patients With CAP in ICU, Should Combination Therapy be Preferred Over Monotherapy?}

In a recent meta-analysis of CAP patients including 28 observational studies,combination antimicrobial regimens including macrolides have been associated with significantly decreased mortality as compared to non-macrolides (RR 0.82; 95\% CI, 0.70-0.97; p = 0.02), along with a trend towards mortality benefit favoring macrolides as compared to fluoroquinolones (RR 0.83; 95\% CI, 0.67-1.03; $\mathrm{p}=0.09) .{ }^{53}$ Combination therapy also resulted in better survival in patients with shock without any significant increase in microbial resistance. ${ }^{54}$ In a matched case-control study of prospectively studied cohorts, combination therapy including macrolides was an independent predictor of survival (OR, 0.19; $95 \%$ CI, $0.07-0.51$ ) in patients with pneumococcal CAP requiring ICU admission. ${ }^{55}$

\section{Evidence Statement}

Empirical combination therapy covering common organisms causing community-acquired pneumonia improves survival without any significant increase in microbial resistance.

\section{Recommendations}

- Patients with CAP requiring ICU admission should initially receive a combination of empirical antimicrobial agents covering common causative organisms (2A).

\section{What Should be the Preferred Combination Therapy for CAP in ICU?}

In a recent meta-analysis of 8 studies ( 1 randomized controlled trial and 7 observational studies), 2273 patients in beta-lactam macrolide arm were compared to 1600 patients in beta-lactam-fluoroquinolonearm; beta lactammacrolide combination was associated with a lower overall mortality as compared to that of beta lactamfluoroquinolone combination (OR, 0.68 ; $95 \%$ CI 0.49-0.94; $\mathrm{p}=0.02)$ along with decreased length of hospital stay (mean difference, -3.05 days; $95 \% \mathrm{CI},-6.01$ to -0.09 ; $\mathrm{p}=0.04) .{ }^{56}$ Aztreonam and fluoroquinolones are effective alternatives to macrolides, however, with undue risk of masking and delaying diagnosis of tuberculosis. ${ }^{57}$ Aztreonam is effective alternative for patients with contraindication to beta lactams.

\section{Evidence Statement}

For patients with severe CAP requiring ICU admission without risk factors for pseudomonal infection, a combination of beta-lactams along with macrolides is better as compared to beta-lactam fluoroquinolone combination in terms of mortality benefit and length of hospital stay.

\section{Recommendations}

- For patients with CAP requiring ICU admission, a non-pseudomonalbeta-lactam (cefotaxime, ceftriaxone, or amoxicillin-clavulanic acid) plus a macrolide (azithromycin or clarithromycin) should be preferred if there are no risk factors for Pseudomonas aeruginosa infection (1A).

- For penicillin-allergic patients, a respiratory fluoroquinolone (levofloxacin, moxifloxacin or ciprofloxacin) and aztreonam may be used (3A).

- If macrolides cannot be used, a fluoroquinolone may be used if there is no clinical suspicion of tuberculosis, after sending sputum or endotracheal aspirate for AFB and Genexpert (3A).

\section{When Should Anti-pseudomonal Cover be Added for CAP in ICU? If Required, Which are the Preferred Antimicrobials for Anti-pseudomonal Cover?}

Age greater than 65 to 70 years,male gender,current smokers, chronic respiratory disease including chronic bronchitis, COPD, asthma or bronchiectasis, cerebrovascular disease, dementia, other chronic neurological disorders, cardiovascular diseases,cirrhosis, immunocompromised states,malignancy, current use of corticosteroids, enteral tube feeding, previous hospital admission, prior antibiotic therapy and severe pneumonia at presentation have been reported as risk factors for CAP due to Pseudomonas aeruginosa in various observational studies. ${ }^{30,42,43,58-62}$ Prior antibiotic therapy has been associated with increased risk of multidrug-resistant pseudomonal infection. ${ }^{60}$

\section{Evidence Statement}

For patients with severe CAP requiring ICU admission, risk factors for infection with Pseudomonas aeruginosa include chronic pulmonary disease (chronic obstructive pulmonary disease, asthma, bronchiectasis), frequent systemic corticosteroid use, prior antibiotic therapy, old age, immunocompromised states, enteral tube feeding, cerebrovascular or cardiovascular disease. Prior antibiotic therapy is a risk factor for multidrug-resistant pseudomonal infection.

\section{Recommendations}

- If $P$. aeruginosa is an etiological consideration, antipneumococcal, antipseudomonal antibiotic (like 
ceftazidime, cefoperazone, piperacillin-tazobactam, cefoperazone-sulbactam, imipenem, meropenemorcefepime) should be used. (2A)

- Combination therapy should be considered with the addition of aminoglycosides or antipseudomonal fluoroquinolones (e.g., ciprofloxacin) (3A).

\section{When Should MRSA Cover be Added to the Empiric Regimen for CAP in ICU?}

Evidence on CAP due to MRSA is limited, and mostly based on small prospective studies, case series or case reports. ${ }^{11-14}$ Asystematic review (81 studies; 7 case series, 71 case reports, 3 observational studies) estimated incidence of MRSACAP to be 0.51 to 0.64 cases per 100,000 population. ${ }^{63}$ MRSA CAP carries high mortality (up to $60 \%$ ). Close contact with a MRSA carrier or patient, preceding influenza infection, prisoners, professional athletes, army recruits, men having sex with men (MSM), intravenous drug abusers, regular sauna users,immunocompromised status (HIV, acute leukemia, ongoing systemic corticosteroid therapy)and those using antibacterial agents before infection have an increased risk of MRSA CAP. ${ }^{63,64}$ Multilobar consolidation, necrotizing consolidation and empyema were also observed in greater proportion of patients with MRSA CAP. ${ }^{13}$ Considering multiple risk factors, relatively low frequency but high morbidity and mortality associated with MRSA $\mathrm{CAP}$, the expert group decided to emphasize on thorough assessment of risk factors for MRSA CAP in ICU, while balancing the Recommendations to guard against blanket MRSA cover for all CAP cases getting admitted to ICU. The most effective antibiotics against MRSA are vancomycin and teicoplanin. Tigecycline is also effective against MRSA; linezolid has also been reported to be effective in MRSA and VRSA pneumonia. ${ }^{8,65}$

\section{Evidence Statement}

Risk factors for MRSA in CAP in ICU include close contact with MRSA carrier or patient, influenza, prisoners, professional athletes, army recruits, men having sex with men (MSM), intravenous (IV) drug abusers, regular sauna users and those with recent antibiotic use. MRSA pneumonia should be suspected after influenza or in previously healthy young patients, if there is cavitation or necrotizing pneumonia, along with the rapid increase of pleural effusion, massive hemoptysis, neutropenia or erythematous rashes. Vancomycin, teicoplanin, linezolid, and tigecycline are effective antibiotics against MRSA.

\section{Recommendations}

- All patients admitted with CAP in ICU should be evaluated for the presence of risk factors associated with MRSA (3A).
- If MRSA is a consideration, empiric vancomycin (1A) or teicoplanin (2A) should be added to the regimen. Linezolid should be used for vancomycin intolerant patients, vancomycin-resistant Staphylococcus aureus (VRSA), or patients with renal failure (1A).

\section{When Should Anaerobic Cover be Added to the Empiric Antibiotic Regimen for CAP in ICU?}

Anaerobic organisms were reported to cause the majority of pulmonary infections associated with lung abscesses (26-100\%), aspiration pneumonia (62-100\%) and empyema (9-76\%) in observational studies. ${ }^{66-74}$ In a recent observational study of 64 patients with CAP, $15.6 \%$ of BAL samples had evidence of anaerobic infection on 16s RNA analysis. ${ }^{75}$ Witnessed aspiration, loss of consciousness due to drug or alcohol overdose, seizures with concomitant gingival disease and dysphagia have been considered as risk factors for anaerobic infection. ${ }^{76}$

\section{Evidence Statement}

Risk factors for aspiration pneumonia in patients admitted with CAP in ICU include dysphagia, altered sensorium, coma, witnessed aspiration, putrid discharge, the presence of lung abscess, empyema or necrotizing pneumonia.

\section{Recommendations}

- Empirical antibiotics with anaerobic coverage should be considered for treatment of CAP in ICU in the presence of clinical risk factors for aspiration or presence of lung abscess, empyema or necrotizing pneumonia. (2A)

\section{Which Antibiotic Should be Preferred for Anaerobic Coverage for CAP in ICU?}

Clindamycin was associated with significantly higher cure rates as compared to penicillin in randomized controlled trials in anaerobic lung infections. ${ }^{71,77}$ In a randomized prospective study of 100 patients with anaerobic lung infections, ampicillin-sulbactam, clindamycin and panipenem-betamiprom had similar clinical efficacy $(\mathrm{p}=0.62)$ and similar duration of treatment $(\mathrm{p}=$ $0.35)$ whereas non-clindamycin group had higher frequency of appearance of MRSA (22.7\% vs. $0 \%$; $p$ $<0.01) .{ }^{78}$ Ampicillin-sulbactam had similar clinical and bacteriologic response to clindamycin with or without cephalosporin in another prospective randomized multicenter study of 70 patients with anaerobic lung infections. ${ }^{79}$ Moxifloxacin demonstrated a similar clinical response to ampicillin-sulbactam in a prospective open-label randomized multicentric study involving 
139 patients with aspiration pneumonia and lung abscess, along with the added advantage of once-daily dosing. ${ }^{80}$ Moxifloxacin was also shown to be superior to levofloxacin-metronidazole combination in terms of clinical cure at 7 weeks $(76.7 \%$ vs. $51.7 \%$; p < 0.05$)$ as well as similar bacteriologic cure (93.3\% vs. $96.4 \%$, $\mathrm{p}>0.05)$ without any significant difference in adverse drug reactions. ${ }^{81}$ Duration of treatment has been reported to be variable. Longer duration of treatment ( 3 to 6 weeks) is required in lung abscesses and empyema. ${ }^{71,79,80}$

\section{Evidence Statement}

Commonly prescribed empirical antibiotics for CAP in ICU such as ampicillin-sulbactam, amoxicillin-clavulanic acid, piperacillin-tazobactam, and carbapenems have excellent anaerobic coverage. Clindamycin and moxifloxacin are effective against aspiration pneumonia and lung abscess caused by anaerobic organisms. Lung abscess and necrotizing pneumonia may require prolonged treatment up to 4 to 6 weeks.

\section{Recommendations}

- Patients with CAP at risk of anaerobic infection should be initiated on antibiotics with anaerobic activity such as amoxicillin-clavulanate, clindamycin or moxifloxa$\operatorname{cin}(1 \mathrm{~A})$.

- Piperacillin-tazobactam or carbapenems can be used for empirical therapy in CAP due to anaerobes if otherwise indicated (3A).

- Duration of treatment should be individualized according to the response and severity of the disease (3A).

\section{What Should be the Optimal Duration of Antibiotics for CAP in ICU?}

On post-hoc analysis of an RCT comparing levofloxacin treatment for 5 days to 10 days, the subgroup with moderate to high severity CAP had similar clinical cure rates (RR 1.07; 95\% CI 0.95 to 1.2). ${ }^{8,82}$ In another study on severe CAP, treatment for more than 7 days did not confer any mortality benefit. ${ }^{83}$ However, this study excluded ICU admission, complicated pneumonia, nonresponding pneumonia or identification of organisms requiring prolonged treatment. Also, Enterobacteriaceae, Pseudomonas, Legionella, and S. aureus were associated with the requirement of prolonged treatment.

\section{Evidence Statement}

For CAP in ICU, there is limited evidence regarding the duration of treatment, with no significant mortality benefit beyond 7 days of antimicrobial therapy in uncomplicated cases. However, CAP due to GNB, Enterobacteriaceae, $P$. aeruginosa, S. aureus bacteremia and L. pneumophila requires prolonged treatment. Necrotizing pneumonia, lung abscess, empyema or extrapulmonary infective complications like meningitis or infective endocarditis also require a longer duration of treatment.

\section{Recommendations}

- Patients with CAP requiring ICU admission should receive antibiotics for 7 to 10 days (2A).

- Patients with CAP due to Pseudomonas or aspiration pneumonia should be treated for 14 days (3A).

- Necrotizing pneumonia due to GNB, MRSA or anaerobes also require treatment for 14 to 21 days (3A)

- Duration of treatment should be individualized according to causative organism, response, the severity of disease and complications (3A).

\section{Should Procalcitonin be Used to Determine the Duration of Antibiotic Administration for CAP in ICU?}

In a recent meta-analysis of 26 trials involving 6708 patients, procalcitonin utilization for antibiotic discontinuation was associated with reduced mortality (adjusted OR $0.83,95 \%$ CI 0.70 to $0.99, \mathrm{p}=0.037) .{ }^{84}$

\section{Evidence Statement}

Serial procalcitonin levels can be used for de-escalation of antibiotics for CAP in ICU, without any increase in mortality or recurrence rates.

\section{Recommendations}

- Procalcitonin levels can be used along with clinical judgment for de-escalation of antibiotics in CAP in ICU in patients treated beyond 5 to 7 days (1A).

\section{Ventilator Associated Pneumonia}

Pneumonia is one of the commonest hospitals acquired infection. Hospital-acquired or nosocomial pneumonia (HAP) is defined as pneumonia that occurs 48 hours (or more) after admission and did not appear to be incubating at the time of admission. Ventilatorassociated pneumonia (VAP) is HAP that develops more than 48 to 72 hours after endotracheal intubation. The previously used term health care-associated pneumonia (HCAP) is currently not in use. ${ }^{85}$ To provide a more uniform and consistent reporting of cases of ventilatorassociated complications, Centre for Disease Control (CDC) has proposed the term ventilator-associated events which include ventilator-associated condition, infection-related ventilator-associated complication, probable VAP and possible VAP. ${ }^{86}$ The incidence of VAP 
varies among different ICUs and depends upon the definition used.In most ICUs, the incidence is around $10-20 \% .{ }^{85}$ Endotracheal intubation compromises the natural barrier between oropharynx and trachea as well as facilitates entry of bacteria into the lungs. ${ }^{87}$ Supine position also facilitates the transfer of contaminated secretions leading to VAP. ${ }^{88}$ VAP is suspected in patients with new or progressive pulmonary infiltrates plus supportive clinical findings suggestive of infection. The diagnosis is made on clinicoradiological findings and is supported by isolation of microorganism from lower respiratory tract sample.Critically ill patients who develop VAP are two times more likely to die as compared with similar patients without VAP. VAP leads to significantly longer ICU length of stay and also incur additional hospital costs. ${ }^{89}$

\section{What are the Common Organisms Causing HAP/VAP in ICU and what is their Antibiotic Susceptibility Pattern?}

The microorganisms implicated in the causation of VAP varies among ICUs. Studies conducted in Western countries demonstrated that majority of VAP episodes are caused by Staphylococcus aureus followed by Pseudomonas aeruginosa. ${ }^{90}$ In a retrospective review of 8474 cases of VAP reported to CDC, Staphylococcus accounted for $24.1 \%$ of cases followed by Pseudomonas (16.6\%) and Klebsiella $(10.1 \%) .{ }^{91}$

Studies from Asia show preponderance of gramnegative organisms as an etiologic agent of VAP. A prospective surveillance study from 73 hospitals in 10 Asian countries from 2008 to 2009 including 2554 cases with HAP or VAP found that Pseudomonas (15.6\%) was most common causative organism followed by Staphylococcus aureus (15.5\%), Acinetobacter spp. (13.6\%) and Klebsiella pneumoniae (12\%). Imipenem resistance of Acinetobacter and P. aeruginosa was $67.3 \%$ and $27.2 \%$ respectively. A large proportion of Acinetobacter (82\%) and $P$. aeruginosa $(42.8 \%)$ were multidrug resistants (MDR) while $51.1 \%$ and $4.9 \%$ were extensively drugresistant (XDR), respectively. The prevalence of MRSA among $S$. aureus isolates was $82.1 \% .{ }^{92}$ Similarly, another retrospective study from Thailand also found A. baumannii (53.4\%) as most common isolate followed by P. aeruginosa (35.2\%) and MRSA (15.1\%). ${ }^{93}$

Multiple studies from Indian ICUs have also shown predominance of gram-negative bacilli (Acinetobacter, and Klebsiella) in VAP. ${ }^{94-96}$ These gram-negative bacilli are often multidrug resistant. A prospective study from Pondicherry showed an incidence of VAP to be $18 \%$ where Pseudomonas and Acinetobacter were common (21.3\%) followed by Staphylococcus (14.9\%). ${ }^{97}$ Another study from Karnataka found A. baumannii to be the commonest organism in both early and late onset VAP followed by Pseudomonas. All isolates of Acinetobacter were resistant to at least three antibiotics (i.e., MDR) and one isolate of Acinetobacter was pan-resistant. ${ }^{98}$ There has been also a rise in carbapenem resistance of Acinetobacter. A study done by Gurjar et al showed that $75 \%$ of patients with VAP due to Acinetobacter were carbapenem resistant. ${ }^{99}$

\section{Evidence Statement}

Ventilator-associated pneumonia (VAP) and hospitalacquired pneumonia (HAP) are commonly caused by aerobic gram-negative bacilli, such as Acinetobacter baumannii, Klebsiella pneumoniae, Pseudomonas aeruginosa, or by gram-positive cocci (Staphylococcus aureus).In Indian ICUs, gram-negative organisms are the most common etiologic agents (i.e., Acinetobacter, and Pseudomonas spp). Most of these pathogens have been found to be multidrug resistant. Frequency of specific MDR pathogens causing HAP and VAP may vary by hospital, patient population, type of ICU patient, and change over time.

\section{What are the Risk Factors for MDR Pathogens in VAP in ICU?}

The incidence of VAP caused by MDR organisms has increased in the last decade and has been associated with increased cost of care, morbidity, and mortality. Data from the early 1980s show that about $50 \%$ of mechanically ventilated patient develop VAP within first 4 days after intubation and were due to non-MDR pathogens. However, several recent studies show no significant difference between causative organisms in both early and late VAP. ${ }^{100}$ Various factors like advanced age $(>60$ years) and prior use of antibiotics have been consistently associated with increased risk of MDR organisms. ${ }^{101,102}$ In a prospective study done by Trouillet et al in 135 cases of VAP, the three variables identified as risk factors for MDR VAP were the duration of mechanical ventilation (7 days or more) and prior use of broad-spectrum antibiotics (third-generation cephalosporins, fluoroquinolones, or imipenem). ${ }^{103}$ Renal replacement therapy and septic shock at admission were also found to be risk factors for MDR VAP. ${ }^{104}$ Higher Acute Physiology And Chronic Health Evaluation II (APACHE II) score on admission, pleural effusion, prior antibiotic treatment, illicit drug use, and tobacco are also found to be risk factors for MDR VAP due to MRSA. ${ }^{105,106}$ Similarly, vasopressor use, trauma, and neurological emergency were identified as additional risk factors for MDR VAP. ${ }^{101}$ Two studies show that systemic corticosteroid therapy has also been implicated as a risk factor for MDR VAP. However, both 
these studies do not mention the dose and duration for which corticosteroid therapy was used. ${ }^{101,107}$

\section{Evidence Statement}

The risk factors for VAP due to MDR organisms include age $>60$ years, duration of mechanical ventilation $\geq 7$ days, prior antibiotic use within 3 months, the presence of severe sepsis or septic shock at the time of VAP, ARDS preceding VAP, renal replacement therapy prior to VAP and systemic corticosteroid therapy.

\section{What Should be the Initial Combination of Empiric Antibiotic Therapy for VAP in ICU?}

Inadequate or inappropriate therapy for VAP has been associated with higher mortality rates. ${ }^{108}$ A Cochrane review included four studies that compared monotherapy to combination antibiotic therapies for VAP. This analysis found no significant difference in the primary endpoint of all-cause mortality and clinical cure rate in intentionto-treat population and clinically evaluable population between monotherapy and combination therapy. Similarly, comparison of combination therapy with optional adjunctive antibiotics (amikacin, vancomycin, linezolid, aztreonam, ceftazidime, and tobramycin) did not find any difference in all-cause mortality, clinical cure rate in intention-to-treat population and clinical cure rate in the clinically evaluable population. No difference in all-cause mortality or clinical cure rate in intention to treat population was found when carbapenems were compared with non-carbapenems; however, carbapenems had a higher chance of clinical cure rate in the clinically evaluable population. This meta-analysis supports the use of a single antibiotic regimen with the understanding that resistance patterns may vary depending upon the local factors. ${ }^{109} \mathrm{~A}$ similar meta-analysis by Infectious Disease Society of America (IDSA) also found no difference between combination therapy versus monotherapy, cephalosporins versus non-cephalosporin regimen, antipseudomonal penicillin versus non-antipseudomonal penicillin regimen and carbapenems versus non-carbapenem regimen. Among aminoglycoside versus non-aminoglycoside regimen, use of aminoglycoside regimen was associated with less chance of clinical response compared to the nonaminoglycoside regimen. When comparing quinolones versus non-quinolone regimen, adverse event rates were less with quinolone regimen [Risk Ratio 0.88 (0.78-0.99) with $95 \% \mathrm{CI}] \cdot{ }^{85} \mathrm{~A}$ meta-analysis by Walkey et al ${ }^{110}$ found that linezolid was not superior to glycopeptide antibiotics for the endpoints of clinical success, microbiological success, and mortality for patients with MRSA nosocomial pneumonia, without any significant difference in adverse events. However, another metaanalysis found more frequent gastrointestinal adverse effects with the use of linezolid. ${ }^{111}$

\section{Evidence Statement}

Use of antibiotic monotherapy and combination therapy for VAP have similar outcomes in patients who are not at risk for MDR pathogens. Commonly used antimicrobial agents include piperacillin-tazobactam, cefepime, levofloxacin, imipenem, and meropenem. Among antimicrobial agents, carbapenems have a higher chance of clinical cure than non-carbapenems. For treatment of VAP due to MRSA, glycopeptides and linezolid have similar clinical success; however, linezolid may be associated with a higher chance of thrombocytopenia and gastrointestinal adverse events.

\section{Recommendations}

- Among patients with VAP who are not at high risk of MDR pathogens and are in ICUs with a low prevalence of MRSA $(<15 \%)$ and resistant gramnegative organisms $(<10 \%)$, single antibiotic active against both MSSA and Pseudomonas is preferred over combination antibiotic (1A).

- Among patients with VAP who are at high risk of MDR pathogens or are in ICU with a high prevalence of MRSA (> 15\%) and resistant gram-negative organisms $(>10 \%)$, an agent active against MRSA and at least two agents active against gram-negative organisms including $P$. aeruginosa is recommended (3A).

- Among patients with VAP who are not at high risk of MDR pathogens and are in ICU with a high prevalence of resistant gram-negative organisms (>15\%) but low prevalence of MRSA $(<10 \%)$, two agents active against gram-negative organism including $P$. aeruginosa is recommended (3A).

- Colistin is not recommended for routine use as an empirical agent in VAP. However, it may be used up front in the ICUs if there is a high prevalence of carbapenem-resistant Enterobacteriaceae (>20\%) (UPP).

- In our country or areas with high endemicity of tuberculosis, use of linezolid may be restricted unless no suitable alternative is available (UPP).

- Fluoroquinolones and aminoglycoside should be cautiously used as monotherapy in VAP in our country as well as in other areas with high endemicity of tuberculosis (UPP).

- In ICU where the distribution of pathogen and antibiotic resistance pattern is known, empiric treatment should be designed accordingly, based upon patient risk factors for MDR pathogens (UPP). 


\section{When to Give Antipseudomonal Drugs for VAP in ICU?}

Antipseudomonal drugs are often started empirically in VAP when the risk factors for Pseudomonas infection are high. In a prospective surveillance study, it was found that the odds of developing P. aeruginosa VAP were 8 times higher in patients with prior $P$. seudomonas colonization than uncolonized patients. ${ }^{112}$ In a multicentre study, the independent risk factors for the presence of $P$. aeruginosa were the duration of hospital stay $\geq 48$ hours before ICU admission, prolonged duration of ICU stay before enrollment $>9$ days (highest quartile) versus ICU stay $\leq 4.8$ days(lowest quartile) ${ }^{113}$ Risk factors of MDR P. aeruginosa include COPD, patients on mechanical ventilation $>8$ days or patients with $>3$ previous hospitalizations, and previous use of antibiotics. ${ }^{114,115}$

\section{Evidence Statement}

Prior use of antibiotics (most consistent association), prolonged duration of mechanical ventilation, and chronic obstructive pulmonary disease (COPD) have been identified as risk factors for MDR P. aeruginosa infection.

\section{Recommendations}

- Empiric treatment should be given to cover Pseudomonas if there are risk factors for MDR Pseudomonas infection (2A).

- In ICUs where gram-negative isolate resistance rate is low $(<10 \%$ gram-negative isolate resistant to the agent being considered for monotherapy) and patients have no risk factors for antimicrobial resistance, one antipseudomonal antibiotic may be given (3A).

- In ICUs where gram-negative isolate resistance rate is high ( $>10 \%$ gram-negative isolate resistant to the agent being considered for monotherapy or not known), two anti-pseudomonal antibiotics from a different class to be given (3A).

\section{What Should be the Duration of Antibiotic Treatment for HAP/VAP?}

Prompt initiation of appropriate antimicrobial therapy is the mainstay of treatment of VAP. Selection of correct antimicrobial agent must be paired with an appropriate duration of therapy in order to optimally treat VAP/HAP. Several studies have evaluated the role of short duration antibiotic treatment in VAP/HAP. A study comparing 8 days therapy to 15 days therapy found no difference in mortality, relapses, mechanical ventilator-free days, organ failure free days and length of ICU stay while short course regimen was associated with more antibiotic-free days. However, gram-negative bacilli ( $P$. aeruginosa) with short course regimen were more likely to have a relapse (40.6\% vs. $25.4 \%)$. ${ }^{116}$ A randomized comparison of antibiotic discontinuation policy(discontinuation group) with treating physician teams policy (conventional group) found lower antibiotic duration in discontinuation group without any difference in a secondary episode of VAP, hospital mortality or ICU length of stay. ${ }^{117}$

\section{Evidence Statement 1}

Short-course regimens for VAP are associated with significantly more antibiotic-free days without any significant difference in the duration of ICU or hospital stay, recurrence of VAP and mortality. Short-course regimens are associated with more recurrences in VAP due to non-fermenting gram-negative bacilli (NF-GNB).

\section{Recommendations}

- Short course (7-8 days) of antibiotic therapy should be used, in the case of VAP with good clinical response to therapy (1A).

- Longer duration (14 days) of antibiotic therapy should be considered, in case of VAP caused by NF-GNBs or is associated with severe immunodeficiency, structural lung disease (COPD, bronchiectasis, and interstitial lung disease), empyema, lung abscess, necrotizing pneumonia, and inappropriate initial antimicrobial therapy (3A).

\section{When Should Anaerobic Cover be Added for VAP and Which is the Preferred Antimicrobial Agent?}

Studies have reported the variable incidence of anaerobic organism isolation in nosocomial pneumonia occurring in mechanically ventilated patients as isolation of anaerobic bacteria requires adequate transport conditions and special growth media. In a retrospective study in 415 patients, factors associated with anaerobic infection were found to be altered level of consciousness and higher simplified acute physiology score (SAPS) ${ }^{119}$ Out of 163 isolates from VAP patients, only one was anaerobic (Veillonella) in a study done by PE Marik et al. ${ }^{120}$ Robert et al evaluated the lower respiratory tract colonization by anaerobic bacteria in ICU patients on prolonged mechanical ventilation. Out of 26 patients, 22 were colonized by at least one bacterial strain and 5 patients developed VAP following colonization, and two were attributable to anaerobic bacteria. ${ }^{121}$

\section{Evidence Statement}

The incidence of anaerobic bacteria as the causative agent of VAP is 2 to $7 \%$. Risk factors for VAP due to anaerobes are altered consciousness, aspiration pneumonitis and high simplified acute physiology score (SAPS). 


\section{Recommendations}

- Empirical antibiotic regimen for VAP should not include coverage for anaerobic organisms routinely (2A).

- In the presence of risk factors for VAP due to anaerobic pathogens, anaerobic antimicrobial coverage should be added in an empirical regimen (2B).

- In patients with risk factors for anaerobic organisms, clindamycin or metronidazole should be added to empirical antibiotics regimen for VAP, if it does not include carbapenems (meropenem or imipenem) or piperacillin-tazobactam in the ongoing empirical regimen (UPP).

\section{When to Give Atypical Cover for VAP and Which is the Preferred Agent?}

Atypical bacteria have been implicated as etiologic agents for VAP; however, no sufficient literature exists to assess the size of their role as a causative agent in VAP. The incidence of atypical bacteria is variable in various studies. A prospective study utilizing polymerase chain reaction (PCR) amplification method found $9(15 \%)$ cases caused by atypical organisms (5 Mycoplasma, 3 Legionella, and 1 Chlamydia). ${ }^{122}$ Another study reported 6 cases of VAP due to Legionella among 26 patients with definite VAP. ${ }^{123}$ M. pneumoniae in 3 patients and C. pneumoniae in 2 patients were diagnosed among $100 \mathrm{VAP}$ cases in a study by Apfalter et al. ${ }^{124}$ The risk factors for Legionella infection include the use of cytotoxic therapy and corticosteroids. ${ }^{125}$ If L. pneumophila is suspected organism for VAP, the combination antibiotic regimen should include a macrolide or a fluoroquinolone rather than an aminoglycoside. ${ }^{126}$

\section{Evidence Statement}

The incidence of atypical bacteria as causative agents of VAP is low (5 to $7.5 \%$ ). Risk factors for VAP due to Legionella are Legionella colonization in hospital water supply, prolonged use of corticosteroids, cytotoxic chemotherapy, elderly, chronic renal failure, previous antibiotic use, granulocytopenia, and poor Glasgow coma score.

\section{Recommendations}

- Empirical antibiotic regimen for VAP should not include coverage for atypical organisms routinely (2A).

- In the presence of risk factors for VAP due to atypical bacterial pathogens, atypical antimicrobial coverage should be added to the empirical regimen (2B).

- The preferred atypical coverage in combination antibiotics regimen is fluoroquinolones (levofloxacin or moxifloxacin) or macrolides (azithromycin or clarithromycin) (UPP).

\section{Can Serum Procalcitonin be used for De-escalation of Antibiotic Therapy in VAP?}

Procalcitonin (PCT) is a polypeptide precursor to hormone calcitonin and is up-regulated from its normal low serum concentration in response to bacterial endotoxin or mediator of bacterial infection. ${ }^{127}$ Measurement of serum PCT has been investigated as a biomarker for the presence and persistence of infection, to guide decisions for initiation, de-escalation, and termination of antibiotic treatment. Delayed initiation of antibiotics in patients with sepsis contribute to increased mortality, while inappropriately prolonged use of antibiotics increases the risk of adverse events, including Clostridium difficile infection, and the development of antibiotic resistance. Various studies have evaluated the role of serum PCT in de-escalation of antibiotics. In a multicentric non-blinded RCT comparing guideline based antibiotic discontinuation with procalcitonin based antibiotic discontinuation, procalcitonin group had higher antibiotic-free days and reduction in the overall duration of antibiotic therapy through the ventilator-free days alive, ICU free days alive, length of hospital stay and mortality on 28 days were similar. ${ }^{128}$ PRORATA trial found that PCT-guided strategy to treat suspected bacterial infection in ICU could reduce antibiotic exposure by 2.7 days with no apparent adverse outcome. ${ }^{129}$ Two meta-analyses have also demonstrated increased antibiotic-free days in PCT-based strategies without negatively affecting the outcome. ${ }^{130,131}$ International guidelines differ on using procalcitonin for antibiotic de-escalation in VAP. American Thoracic Society guidelines suggest using PCT plus clinical criteria to guide the discontinuation of antibiotic therapy rather than clinical criteria alone. ${ }^{85}$ In contrast, European Respiratory Society (ERS) guidelines do not recommend the routine measurement of serial serum PCT levels to reduce the duration of antibiotic course in patients with HAP or VAP when the anticipated duration is 7 to 8 days although panel mention that they believe in measurement of serial serum PCT levels together with clinical assessment in specific clinical circumstances (such as severely immunocompromised patients, drug-resistant pathogens-NF-GNB, and initial inappropriate therapy). ${ }^{132}$

\section{Evidence Statement}

Use of procalcitonin to guide de-escalation of antibiotic treatment in patients with VAP is effective in reducing antibiotic exposure, without an increase in the risk of mortality or treatment failure. 


\section{Recommendations}

- Serum procalcitonin may be used to guide the de-escalation of antibiotics in VAP when the anticipated duration of therapy is $\geq 7-8$ days (1B).

- Serum procalcitonin levels (together with clinical response) should be used for de-escalation of antibiotic therapy in VAP in specific clinical conditions (severely immunocompromised patients, drugresistant pathogens-NF-GNB, initial inappropriate therapy) (3A).

\section{How to Approach a Patient of Non-responding VAP?}

Non-responding VAP or treatment failure in VAP is defined as the lack of improvement in clinical parameters (48-72 hours) with or without persistence of the infecting microorganism from the appropriate sample. ${ }^{133,134}$ Various clinical parameters such as the white blood cell count, measures of oxygenation and core temperature have been used in studies to define the normal pattern of resolution of HAP. In a prospective cohort study assessing the resolution of VAP, it was found that temperature normalizes within a median of 3 days and the ratio of arterial oxygen partial pressure to fractional inspired oxygen (PaO2/ FiO2 ratio) improves by 2 days. ${ }^{135}$ Another study evaluated the bacteriological and clinical efficacy of microbiological treatment of VAP among 76 VAP cases and demonstrated that appropriate antimicrobial therapy for VAP results in the control of the initial infection in $88 \%$ of the patients after day 3 of treatment. ${ }^{136}$ There are many implicated causes for non-resolution of VAP. These include wrong diagnosis (such as collapse, mass or pleural effusion), inappropriate initial treatment, delayed initiation of treatment, superinfection, the concomitant focus of infection or associated complications in the form of lung abscess, empyema or drug fever. ${ }^{137,138}$

\section{Evidence Statement}

Re-evaluation at 48 to 72 hours after the initial diagnosis of VAP is the most suitable time. By then the results of the initial microbial investigation are usually available, and treatment modification can be done. Evaluation of treatment response for VAP should be on the basis of clinical, laboratory, radiograph and microbiological results. Factors associated with treatment failure in VAP includes host factors (advanced age, immunosuppressed, chronic lung disease, ventilator dependence), bacterial factors (drug-resistant pathogens, opportunistic pathogens), therapeutic factors (inappropriate antibiotics, delayed initiation of therapy, insufficient duration of therapy, suboptimal dosing, inadequate local concentration of drugs), complications of initial VAP episode (lung abscess, empyema), other non-pulmonary infections or non-infectious mimics of pneumonia.

\section{Recommendations}

- Non-responding VAP should be evaluated for noninfectious mimics of pneumonia, unsuspected or drug-resistant pathogens, extrapulmonary sites of infection, and complications of pneumonia or its therapy and diagnostic testing should be directed to whichever of these causes is likely (2A).

\section{Catheter-related Bloodstream Infections (CRBSI)}

Intravascular catheters are integral in the management of critically ill patients, especially those who require long-term medical care. They are most commonly used to access the vascular system for the delivery of medication, parenteral nutrition, a collection of blood samples and hemodynamic monitoring. ${ }^{139}$ CRBSI is defined as the presence of bacteremia originating from an intravenous catheter is a common complication leading to morbidity, mortality and adds to the cost of ICU stay. It is also the most common cause of nosocomial bacteremia in ICUs. ${ }^{140}$

\section{Definition and Diagnosis}

Catheter-related Bloodstream Infections (CRBSI) is defined as bacteremia or fungemia in a patient who has an intravascular device and one positive blood culture result obtained from the peripheral vein, clinical manifestations of infection (e.g., fever, chills, and/or hypotension), and no apparent source for bloodstream infection (other than the catheter). One of the following should be present, i.e., a positive result of semi-quantitative [ $>15$ colony forming units (CFU) per catheter segment] or quantitative $\left(>10^{2}\right.$ CFUs per catheter segment) catheter culture, whereby the same organism is isolated from a catheter segment and a peripheral blood culture; simultaneous quantitative cultures of blood with a ratio 13:1 of CFU per milliliter of blood (catheter $v s$. peripheral blood); differential time to positivity (growth in a culture of blood obtained through a catheter hub is detected by an automated blood culture system at least 2 hours earlier than a culture of simultaneously drawn peripheral blood of equal volume). ${ }^{141}$ Catheter tip colonization (CC) is defined as significant growth of a microorganism (>15 colony-forming units) from the catheter tip culture. ${ }^{141}$ CRBSI rates are expressed as CRBSI rate per 1000 central line days. However, the suspicion of CRBSI arises in a patient using any intravascular catheter especially central venous catheter (CVC) who develops new-onset fever or chills, unexplained hypotension without any other localizing signs of infection. ${ }^{140}$ 


\section{What is the Incidence of Catheter Colonization and CRBSI?}

Based on United States (US) data from National Nosocomial Infections Surveillance (NNIS) from 1990 to 1994, the CRBSI incidence (per 1000 catheter days) was 4.3 for respiratory intensive care units (RICU), 4.6 for medical-surgical ICUs, 7.3 for trauma ICUs and 12.2 for burn units. ${ }^{142}$ Data from NNIS from January 1992 through June 2004 showed that the median rate of CRBSI in ICUs of all types ranged from 1.8 to 5.2 per 1000 catheter days, ${ }^{143}$ whereas another survey in 2010 showed the mean incidence up to 1.76 per 1000 catheter days, suggesting a decreasing trend. ${ }^{144}$

Data from Extended Prevalence of Infection in Intensive Care Study (EPIC 2) showed an overall point prevalence of 4.7 per 1000 catheter days. ${ }^{145}$ A prospective observational study by Lorente et al showed the incidence of CC as $6.04 \%$ and of CRBSI to be 2.79 per 1000 catheter days. ${ }^{146}$ Other studies have shown the global incidence of CC to be 1.4 to $20 \%$ while that of CRBSI to be 2.4-12.5 per 1000 catheter days. ${ }^{147-150}$ Majority of these studies have shown CVCs as the commonest cause for CRBSIs. The data from India suggest a higher incidence of CC and CRBSI. In a study by Mittal et al CC was found in $59 \%$ catheters with CRBSI rate of 9.5 per 1000 days. ${ }^{139}$ Others have shown the incidence of CC as 18 to $42 \%$ while of CRBSI is 1 to 16.1 per 1000 catheter days. ${ }^{151,152}$

\section{Evidence Statement}

The global incidence of CC ranges from 1.4 to $19.4 \%$ whereas CRBSI incidence ranges from 2.4 to $12.5 \%$. The incidence of CC is higher in Indian ICUs ranging from $18 \%$ to as high as $59 \%$, whereas the incidence of CRBSI is up to 16.1 per 1000 catheter days.

\section{What are the Risk Factors for CRBSI?}

The incidence of CRBSI varies considerably according to various factors such as the type of catheter (single or multi-lumen), duration of indwelling catheters, the frequency of catheter manipulation, and patient-related factors such as age, underlying disease, and severity of illness. In a retrospective study in 73 events of CRBSI, major risk factors found were advanced age, long-term indwelling catheter, parenteral nutrition, diabetes mellitus (DM), and APACHE II score $>23$, and more than three underlying diseases. Multivariate analysis showed that an APACHE II score $>20$ and more than three underlying diseases were independent factors associated with CRBSI occurring within 14 days of CVC insertion. ${ }^{153}$ Duration of the catheter is an important parameter and catheter duration $>14$ days is an independent risk factor for CRBSI. ${ }^{147,150,154-157}$ The risk for CRBSI is higher when the interval time for dressing change is longer than 48 hours irrespective of the dressing material (permeable or semi-permeable). ${ }^{149}$ Use of transparent dressings, regular change of dressings, total parenteral nutrition, and use of three-way cannulas have not been consistently associated with increased risk for CRBSIs. ${ }^{149,154}$ Regarding hemodialysis (HD) catheters, prospective data by Caylan et al in 248 patients with HD catheters have shown acute renal disease, administration of antibiotics at the time of catheterization, insertion in the femoral vein, emergency situation for catheter insertion, high number of catheter manipulation, and inadequate hand hygiene prior to catheter manipulations as risk factors of CRBSI. ${ }^{158}$ Catheter-related candidemia should be suspected in patients with any of the following risk factors: total parenteral nutrition, prolonged use of broad-spectrum antibiotics, hematologic malignancies, and receipt of bone marrow or solid-organ transplant, femoral catheterization, or colonization due to Candida species at multiple sites. ${ }^{141}$

\section{Evidence Statement}

Longer indwelling catheter duration, immunosuppression, diabetes mellitus, sepsis at the time of insertion, multilumen catheters and APACHE $>23$ are important risk factors for CRBSI. APACHE at admission, renal failure, central venous catheterization, and steroid therapy are important risk factors for fungal CRBSI.

\section{What are the Common Organisms Causing CRBSI and their Antibiotic Susceptibility?}

Apart from the severity of the patient's clinical disease and risk factors for infection, the initial choice of antibiotics will also depend on the likely pathogens and their susceptibility patterns. According to the available literature, certain organisms should always be considered, apart from taking local epidemiology into account. National Nosocomial Infections Surveillance (NNIS) survey of nosocomial infections from 1990 to 1999 showed coagulasenegative Staphylococcus (CONS), Staphylococcus aureus and Enterococcus as common organisms while Candida albicans accounted for $5 \%$ of the CRBSI. Alarge proportion of CONS isolates were methicillin resistant, and the incidence of MRSA and vancomycin-resistant Enterococcus (VRE) was $54.5 \%$ and $25.9 \%$ respectively. ${ }^{142}$ According to NNIS 2004 data, $87 \%$ of CRBSI were monomicrobial, out of which $65 \%$ were gram-positive organisms, $25 \%$ were gram-negative organisms and $9.5 \%$ were fungi, with CONS, Staphylococcus, and Candida being the common organisms. ${ }^{159}$ During this period, there was $12 \%$ increase in VRE and 11\% increase in MRSA. There was a marked increase in ESBL producing 
Klebsiella with $47 \%$ increase in overall incidence. The proportion of CRBSI due to gram-negative organisms like Pseudomonas, Acinetobacter and Klebsiella is also on rising trends according to recent studies. In a recent observational study, CRBSIs due to Pseudomonas and Acinetobacter were $22.2 \%$ and $20 \%$ respectively. ${ }^{147}$ This rise in gram-negative organisms has been found in various studies from India as well. ${ }^{152,153,160,161}$ In Indian ICUs the MRSA incidence ranges from 30 to $87 \%$, and that of VRE is as high as $25 \% .{ }^{160,161}$ Incidence of ESBL producing organisms has also increased with some studies showing all isolates to be ESBL producing. ${ }^{162}$ The proportion of CRBSI caused by fungi varies among different studies and usually ranges from 4.4 $\%$ to $20 \%$ and mostly was due to Candida albicans. ${ }^{161,163}$ However, a prospective observational study from 27 Indian ICUs found Candida tropicalis (41.6\%) as the most common cause of fungemia followed by Candida albicans (20.9\%) and Candida parapsilosis (10.9\%). Majority of C. tropicalis isolates were sensitive to amphotericin B (99.0\%), azoles (90.1\%), fluconazole (97.4\%) and echinocandins (94.2\%). ${ }^{164}$

\section{Evidence Statement}

Coagulase-negative staphylococci (CONS), S. aureus, Enterococcus, and Candida species are the common organisms accounting for the majority of the CRBSIs. A large proportion of Staphylococcus aureus and CONS are methicillin resistant ranging from 11 to $87 \%$. There is an increased incidence of CRBSI due to gram-negative organisms (most of which are ESBL producers) and Candida especially the non-albicans Candida.

\section{What is/are the Empiric Antibiotic(s) of Choice for CRBSI in ICU?}

Empiric treatment, when indicated, should provide coverage against the most frequent organisms causing CRBSI, i.e. gram-positive as well as gram-negative organisms. Vancomycin, teicoplanin, and linezolid are considered the initial drugs of choice for empiric treatment for gram-positive organisms as the incidence of methicillin resistance is high among CONS and $S$. aureus. A recent meta-analysis by $\mathrm{Li}$ et al included 7 RCTs comparing linezolid with vancomycin in 5376 patients with MRSA. ${ }^{165}$ The clinical cure rate in linezolid group was higher than that of vancomycin group after treatment (OR 1.85; 95\% CI: 1.33-2.59, p <0.001) and at follow-up (OR 1.49; 95\% CI: 1.17-1.91, p = 0.001). However, linezolid monotherapy has not been recommended for empirical treatment of patients with suspected CRBSI. ${ }^{166}$ Teicoplanin is a safe and effective alternative to vancomycin considering the lesser toxicity and once daily dosing. ${ }^{167}$ Quinupristin-dalfopristin and daptomycin may be alternative drugs effective in MRSA bacteremia and enterococci showing comparable results with vancomycin in RCTs. ${ }^{168,169}$ Dalbavancin is another drug belonging to same class as vancomycin and when used in weekly doses, has shown higher success rate than vancomycin for treatment of CRBSI. ${ }^{170}$ For treatment of VRE, a significantly lower mortality rate and trend towards better clinico-microbiologic response has been seen using linezolid as compared to quinupristin-dalfopristin. ${ }^{171}$ Apart from gram positive coverage, an antimicrobial agent with activity against aerobic gram-negative bacilli should be added to the empiric coverage of CRBSI. The appropriate options include aminoglycosides, aztreonam, third-generation cephalosporins with antipseudomonal activity, fourth-generation cephalosporins, piperacillintazobactam or quinolones. ${ }^{141}$ In patients with risk factors for candidemia empiric treatment against Candida is sometimes considered. Caspofungin and fluconazole have equal cure rates in culture positive Candida infections with no difference in mortality as compared to amphotericin B. ${ }^{172,173}$

\section{Evidence Statement}

Vancomycin, teicoplanin, linezolid, and daptomycin are effective in the treatment of CRBSI due to MRSA and MR-CONS. Fourth-generation cephalosporin, carbapenem or beta-lactam/beta-lactamase combination like piperacillin/tazobactam and aminoglycosides might be used for gram-negative organisms causing CRBSI. Caspofungin and fluconazole are equally effective as amphotericin-B for treatment of candidemia.

\section{Recommendations}

- Empirical antibiotic regimen for CRBSI should include coverage for both gram-positive and gram-negative organisms (2A).

- Vancomycin or teicoplanin is the recommended firstline drug for the empiric treatment of CRBSI for MRSA and MR-CONS while linezolid and daptomycin are good alternative agents (2A).

- Empiric coverage for gram-negative bacilli should include a fourth-generation cephalosporin, a carbapenem, or a $\beta$-lactam/ $\beta$-lactamase inhibitor combination, with or without an aminoglycoside. (UPP)

- An echinocandin or fluconazole should be used as empirical antifungal agents for the treatment of suspected central line-associated candidemia (2A).

\section{What Should be the Duration of Antibiotic Treatment for CRBSI?}

Optimum duration of antibiotic treatment to the bare minimum required to treat infections is a reasonable approach to reduce the prevalence of resistance to 
antibiotics. No significant differences in clinical cure, microbiologic cure and survival were detected among bacteremic patients receiving shorter ( 5 to 7 days) versus longer duration (7 to 21 days) of antibiotic therapy in a meta-analysis. ${ }^{174}$ There was 5 to $10 \%$ relapse rate after short-course therapy for Staphylococcus aureus catheterassociated bacteremia suggesting that short-course therapy is acceptable for uncomplicated infections. In case of complicated $S$. aureus infections like infective endocarditis, longer duration (4 to 6 weeks) of treatment is required. Studies have shown similar response irrespective of duration of therapy in gram-negative infections as well. Regarding the duration of empirical antifungals for CRBSIs, there has been no comparative studies but based on the consensus, approximately 14 days of empirical antifungals are recommended.

\section{Evidence Statement}

Short duration (<14 days) of antibiotics is as effective as longer duration (>14 days) for uncomplicated Staphylococcus aureus bacteremia. Complicated bacteremia due to $S$. aureus or those associated with endocarditis should receive longer duration. For gram-negative bacteremia, seven days of antibiotics are sufficient. In responding patient with uncomplicated CONS infection, 5 to 7 days therapy is considered optimum. Minimum 14 days treatment with antifungals is required for fungal CRBSI.

\section{Recommendations}

- Minimum 2 weeks antibiotics should be given for uncomplicated and 4 to 6 weeks for complicated Staphylococcus aureus CRBSI and infective endocarditis (2A).

- Minimum 7 days of antibiotics should be given for gram-negative CRBSI (2A).

- Five to seven days antibiotics are recommended for CONS bacteremia (3A).

- For suspected fungal CRBSI, antifungal therapy for at least 14 days is recommended (UPP).

\section{Empirical Antibiotics for Urinary and Urogenital Sepsis in ICU}

Urogenital infections in patients in the ICU include urinary tract infection (UTI) and prostatitis in males. The clinical spectrum of UTI includes asymptomatic bacteriuria and funguria to pyelonephritis, and urosepsis with or without obstructive uropathy. Urinary tract infections are the fourth most common type of healthcare-associated infection. ${ }^{175}$ UTI additionally account for more than $12 \%$ of infections reported by acute care hospitals. About 12 to $16 \%$ of hospitalized adults have indwelling urinary catheter at some time during their hospitalization. Each day the indwelling urinary catheter is in place, there is a 3 to $7 \%$ increased risk of acquiring a catheter-associated urinary tract infection. ${ }^{176}$ UTIs in ICU have different microbiology and higher resistance rates than UTI occurring outside ICU. Urinary tract infection is defined as significant bacteriuria in a patient with symptoms or to the urinary no alternate source. Significant bacteriuria in a patient without symptoms or signs attributable to the urinary tract is defined as asymptomatic bacteriuria.

Catheter-associated urinary tract infection (CAUTI) is defined as an infection occurring in a person whose urinary tract is currently catheterized or has been catheterized within the previous 48 hours with urethral, suprapubic or intermittent catheterization. It is characterized by symptoms and signs suggestive of UTI with no other obvious source and a urine sample (from the urinary catheter, or midstream urine for catheter duration less than 48 hours) demonstrating more than $1000 \mathrm{CFU}$ per $\mathrm{mL}$. On the other hand, catheter-associated asymptomatic bacteriuria refers to patients with urethral, suprapubic or intermittent catheterization with urine culture positivity $(>100000 \mathrm{CFU} / \mathrm{mL})$ without any signs or symptoms attributable to UTI. According to CDC, CA-UTI is defined as a UTI in patients with an indwelling urinary catheter that had been in place for $>2$ days on the date of event (day of device placement =D1) and was either present for any portion of the calendar day on the date of event or removed the day before the date of event. The patient should have at least one of the following signs or symptoms: fever, supra-pubic tenderness, costovertebral angle pain or tenderness, urinary urgency, urinary frequency, and dysuria along with urine culture with no more than two species of organisms identified at least one of which is a bacterium of $\geq 10^{5}$ $\mathrm{CFU} / \mathrm{mL}^{177}$

\section{What is the Incidence of UTI in ICU? What are the Common Organisms and Risk Factors for UTI in ICU?}

The incidence of UTI ranges from 5 to 23 per 1000 catheter days as reported from various observational studies from the West. ${ }^{178-183}$ In an observational study, Tay et al from Singapore reported the incidence of UTI from mixed ICU to be $13.7 \%$ in patients admitted for more than 48 hours, with the incidence of Candida being about 34\% ${ }^{184}$ The organisms causing UTI were Klebsiella (7\%), E. coli (7\%), polymicrobial $(37 \%)$ and others $(7 \%)$. Female gender, prior antibiotic exposure, duration of ICU and urinary catheter were identified as risk factors for UTI. In a prospective observational study from China, Xie et al ${ }^{185}$ reported the incidence of UTI to be 25.5 per 1000 catheter days. Fungi 
$(21.3 \%)$ were the most common cause of UTI followed by infection with E. coli (17.02\%) and Pseudomonas $(10.64 \%)$. The risk factors for CA-UTI were the duration of the catheter for $>7$ days, benign prostatic hypertrophy and $>5$ days antibiotic duration. Pseudomonas showed absolute resistance to ciprofloxacin, amikacin, ceftazidime, and meropenem. A prospective study by Leone et al reported an incidence of UTI to be $9.6 \%$. The common organisms isolated were E. coli $(39 \%)$, Pseudomonas (22\%) and Enterobacter (15\%). ${ }^{186}$ Duration of catheterization, length of ICU stay, advanced age, female gender, and disease severity score were identified as risk factors for CA-UTI. Similar findings were reported by various studies from western world. ${ }^{187-190}$ In the ENVIN registry, gram-negative bacteria were responsible for more than half of the cases of UTI (56.7\%) with E. coli being the commonest organism isolated $(26.7 \%)$. Fungal infection was second most common (25.4\%) with Candida albicans as most common fungus isolated. ${ }^{191}$ In a prospective study by Agarwal et $\mathrm{al}^{192}$ from Northern India, the organisms causing UTI in ICU included Acinetobacter (34.8\%), Pseudomonas (23.8\%) and E. coli $(15.2 \%)$. Length of ICU stay, renal failure and total parenteral nutrition (TPN) were reported as risk factors for UTI. In a prospective observational study by Habibi et $\mathrm{al}^{193}$ including patients with greater than 48 hours of ICU stay, most common causes of UTI were Candida spp. (90\%) followed by Pseudomonas (14\%) and E. coli $(10 \%)$. Increased ICU stay and catheterization were identified as risk factors for UTI. Gupta et al ${ }^{194}$ reported the incidence of UTI in patients admitted in ICU to be $28 \%$. E. coli was the most common organism responsible for UTI (30.8\%). Longer ICU stay, catheterization and prior antibiotics use were identified as risk factors for UTI. In a retrospective review by Sahu et $\mathrm{al}^{195}$ incidences of UTI reported was $6.9 \%$. Identified risk factors included longer ICU stay and catheterization.

\section{Evidence Statement}

The incidence of CA-UTI ranges from $5-30 \%$ of all ICU admissions. The most common organism causing UTI in ICU are gram-negative bacteria (E. coli, Klebsiella) and fungi (especially Candida). Risk factors for UTI in ICU include the duration of catheterization, length of ICU stay, prior antibiotic use, higher disease severity score, and female gender.

\section{What is the Empirical Antimicrobial Agent of Choice for Treating UTI in ICU?}

A systematic review and meta-analysis by Vardakas et $\mathrm{al}^{196}$ included 21 studies and 1584 patients with ESBL producing Enterobacteriaceae bacteremia. He compared the mortality associated with carbapenems and alternative antibiotics (beta-lactams/beta-lactamase inhibitors) for the treatment of patients with ESBL-positive Enterobacteriaceae bacteremia. No statistically significant differences in mortality were found between carbapenems and beta-lactams/beta-lactamase inhibitors administered as a definitive or empirical treatment for UTI.

In an observational study on gram-negative UTI in hospitalized patients, all isolates were susceptible to carbapenems, with 70 to $80 \%$ susceptible to fluoroquinolones, aminoglycosides, and cefepime. Organisms were resistant to amoxicillin, amoxicillinclavulanic acid and co-trimoxazole. Gram-negative Enterobacteriaceae was also resistant to the second and third generation cephalosporins. ${ }^{197}$ Another prospective study reported an increase in the frequency of gram-negative Enterobacteriaceae and S. aureus in catheter-associated nosocomial UTI over 10 years, with high sensitivities to amikacin, imipenem, and piperacillin-tazobactam $(72.0 \%, 77.5 \%$, and $76.1 \%$, respectively). Lower susceptibility to thirdgeneration cephalosporins and ciprofloxacin (55.2\% and $45.0 \%$ respectively) were reported. Gram-positive organisms showed high susceptibility to teicoplanin and vancomycin (91.1\% and $87.9 \%$ respectively) and low susceptibility to ampicillin and ciprofloxacin (24.1\% and $25.5 \%$ respectively). ${ }^{198}$ Habibi et al ${ }^{193}$ from northern India reported the antibiotics resistance pattern of gram-negative bacteria causing UTI. In this study, the bacteria were resistant to ceftazidime and netilmicin. Cefoperazone-sulbactam resistance was least common among gram-negative organisms. Sahu et $\mathrm{al}^{195}$ reported the least resistance to tigecycline, colistin, and carbapenems among the gram-negative Enterobacteriaceae. One study reported antibiotic susceptibility pattern in gram-negative Enterobacteriaceae and most of the isolates were susceptible to carbapenems, amikacin and levofloxacin. ${ }^{199}$ In a RCT, three antibiotics piperacillin-tazobactam, cefepime and ertapenem were compared in terms of clinical and microbiological cure rate and 28 days mortality for treatment of ESBL producing E. coli. Both cure rates were high for piperacillin-tazobactam and ertapenem. Cefepime was found least effective in terms of both cure rate and prevention of mortality. ${ }^{200}$ In a prospective study, $89.2 \%$ of urinary culture isolates were sensitive to fosfomycin; $89.2 \%$ of gram negative bacilli including Enterobacteriaceae were also susceptible. ${ }^{201}$ Patel et $\mathrm{al}^{202}$ evaluated in vitro activity of fosfomycin against urinary tract Enterobacteriaceae; $79.16 \%$ of the isolates were susceptible to fosfomycin with $92 \%$ susceptibility in ESBL producing Enterobacteriaceae and $72.34 \%$ in 
carbapenem resistant Enterobacteriaceae (CRE). MDR Enterobacteriaceae with diverse resistance mechanisms, including ESBL and CRE were found to be susceptible to fosfomycin. ${ }^{5}$

\section{Evidence Statement}

There has been a trend towards increasing prevalence of extended-spectrum beta-lactamase producing gramnegative bacteria in the urinary cultures of catheterassociated UTI. Aminoglycosides, beta-lactams along with a beta-lactamase inhibitor as well as carbapenems and fosfomycin have good efficacy in catheter-associated UTI. The susceptibility for fluoroquinolones is decreasing over time among organisms isolated from nosocomial UTI. Candida species isolated from the patients with UTI show sensitivity to fluconazole.

\section{Recommendations}

- Initial choice of antibiotics should cover for ESBL producing gram-negative organisms and includes aminoglycosides, beta-lactam along with a betalactamase inhibitor or carbapenems (2A).

- In the initial empirical regimen for UTI, antibiotics against gram-positive organisms are not recommended (3A).

- In appropriate clinical settings, antifungals should be considered in the empirical regimen (3B).

\section{Acute Infective Diarrhea, Antibiotic-Induced Diarrhea, and Clostridium difficile Associated Diarrhea in the ICU}

Diarrhea is defined as the passage of more than three liquid stools in a day. ${ }^{206}$ Nosocomial diarrhea is defined as one which arises after 3 or more days of admission to the hospital. ${ }^{207} \mathrm{Up}$ to $30 \%$ of patients in hospital develop nosocomial diarrhea and majority of which have non-infectious etiology. Among infectious causes, Clostridium difficile associated diarrhea is the most common. ${ }^{208}$ Overall the incidence of diarrhea in intensive care unit varies between 15 to $40 \%$ in different studies where most cases have a non-infectious or multifactorial etiology. ${ }^{209}$

\section{Etiology of Diarrhea in the ICU}

Non-infectious etiologies of diarrhea are commoner in ICU, including enteral feeding, stool impaction and drugs (laxative, prokinetics, histamine antagonists, potassium supplements). ${ }^{210}$ Other factors such as sepsis, antibiotic therapy, and hypoalbuminemia increase the likelihood of diarrhea. ${ }^{211}$ Clostridium difficile is the most common infectious agent associated with diarrhea in the ICU. ${ }^{212}$ Infectious etiology is suspected if diarrhea is associated with fever, leukocytosis, vomiting, severe abdominal pain, mucus or blood in the stool. ${ }^{213}$ Clinical presentation may range from mild infection to life-threatening illness with the pseudo-membrane formation, toxic megacolon, colonic perforation, sepsis or even death. ${ }^{212}$ The American College of Gastroenterology (ACG) have proposed a severity scoring system for Clostridium difficile infection. ${ }^{214}$

\section{Diagnosis of Acute Infective Diarrhea in the ICU}

Clostridium difficile accounts for the majority of infectious diarrhea in the ICU. Most commonly employed screening test is enzyme immunoassay (for Toxin A and B). ${ }^{215}$ Gold standard for diagnosis remains cytotoxin neutralization assay (CCNA) and toxigenic culture, with the latter being more sensitive. ${ }^{215}$ Other diagnostic tests include stool glutamate dehydrogenase and polymerase chain reaction techniques. As per Clostridium difficile infection (CDI) severity index, $\mathrm{CDI}$ is defined as severe and complicated if it is associated with any of the following, i.e., hypotension, fever $\left(\geq 38.5^{\circ} \mathrm{C}\right)$, ileus or significant abdominal distension, mental status changes, leucocytosis $(\geq 35,000$ cells / $\left.\mathrm{mm}^{3}\right)$, leucopenia $\left(<2,000\right.$ cells $\left./ \mathrm{mm}^{3}\right)$, lactic acidosis $(>2.2 \mathrm{mmol} / \mathrm{l})$ or end organ failure. Severe disease refers to CDI with hypoalbuminemia $(<3 \mathrm{~g} / \mathrm{dL})$ along with either abdominal tenderness or leucocytosis (WBC $\geq 15,000$ cells $/ \mathrm{mm}^{3}$ ). Mild to moderate disease refers to CDAD not satisfying above criteria. Clostridium difficile infection (CDI) is a leading cause of hospital-associated gastrointestinal illness and places a high burden on our health-care system. Patients with CDI typically have extended lengths-of-stay in hospitals, and CDI is a frequent cause of large hospital outbreaks of disease. ${ }^{216}$

\section{What are the Common Organisms Causing Acute Infective Diarrhea in the ICU?}

In a large prospective study, it was reported that infectious etiologies accounted for $9.2 \%$ of cases of acute diarrhea in a mixed general intensive care unit. ${ }^{217}$ Clostridium difficile was the most common infective cause accounting for 97 out of the 112 patients in the above study. ${ }^{217}$ In Indian studies, the incidence of CDI was around 16 to $17 \% .{ }^{218,219}$ Other organisms include Pseudomonas aeroginosa and Staphylococcus which have been associated with sporadic outbreaks of diarrhea in the intensive care unit. 220,221 Viruses are another important cause of infective diarrhea in ICU. Norovirus was isolated in $5.7 \%$ cases in one study. ${ }^{217}$ Outbreaks of viral diarrhea due to norovirus have also been reported in ICU settings. ${ }^{222}$

\section{Evidence Statement}

The incidence of diarrhea in the ICU ranges from 12.9 to $38 \%$. Majority of the cases of diarrhea in ICU are non- 
infectious in etiology. Clostridium difficile is responsible for the majority of infectious cases of diarrhea in ICU.

\section{What are the Empirical Antibiotics of Choice for Treating Acute Infective Diarrhea in the ICU?}

There is a lack of studies that evaluate the use of empirical antibiotics in patients with diarrhea in the ICU setting. In a prospective study evaluating the utility of metronidazole in presumptive Clostridium difficile diarrhea involving 70 patients, $18(25 \%)$ were subsequently proven to have Clostridium difficile associated diarrhea (CDAD) whereas $49(68 \%)$ patients had no identifiable cause. Patients who had CDAD had significant improvement in symptoms as compared to those without it. ${ }^{223}$ The American College of Gastroenterology guidelines assert that patients with diarrhea in the ICU who have a strong pre-test suspicion of CDI should receive empirical treatment pending the results of laboratory testing, and even in patients with negative testing, as the negative predictive value of existing tests for CDI is insufficiently high to rule out the infection. ${ }^{214}$

\section{Evidence Statement}

Empirical use of metronidazole in patients with diarrhea suspected due to Clostridium difficile in ICU setting results in significant symptomatic improvement.

\section{Recommendations}

- We recommend that empiric metronidazole be used for therapy of patients with acute diarrhea in the ICU with suspected Clostridium difficile infection (3A).

\section{What are the Risk Factors for the Development of CDI or CDAD?}

Various factors associated with increased risk of CDI include prior antibiotic use, advanced age, prolonged ICU or hospital stay, immunosuppression, proton pump inhibitor use, and enteral feeding. In a recent metaanalysis, previous antibiotic use of second-generation cephalosporins (OR 2.23, 95\% CI 1.47-3.37), thirdgeneration cephalosporins (OR 3.20, 95\% CI 1.80-5.71), fourth-generation cephalosporins (OR 2.14, 95\% CI 1.30-3.52), carbapenems (OR 1.84, 95\% CI 1.26-2.68), clindamycin (OR 2.86, 95\% CI 2.04-4.02), co-trimoxazole (OR 1.78, 95\% CI 1.04-3.05), fluoroquinolones (OR 1.66, 95\% CI 1.17-2.35) and penicillin combinations (OR 1.45, 95\% CI 1.05-2.02) increased the risk of CDAD. . $^{224-234}$

Advanced age has been shown to be associated with increased incidence of CDI. ${ }^{219,235-237}$ Other risk factors for CDI/CDAD include longer ICU stay, enteral feeding,prolonged mechanical ventilation, and immunosuppression. ${ }^{212-214,218,225,236-241}$ Proton pump inhibitors (PPI) have been shown to be independent risk factor for CDAD, possibly due to elevated gastric $\mathrm{pH}$ accelerating conversion of $C$. difficile spores to vegetative forms. ${ }^{219,242-245}$

\section{Evidence Statement}

Risk factors for the development of CDI include prior antibiotic therapy, advanced age, prolonged ICU/hospital stay, immunosuppression, proton pump inhibitors, and enteral feeding. Cephalosporins, clindamycin, fluoroquinolones, carbapenems, and penicillin derivatives are the commonly implicated antibiotics for CDAD/CDI.

\section{What is the Recommended Treatment for CDI/CDAD: Which Antibiotics and Duration? Should Offending Antibiotics be Stopped? What is the role of Probiotics in the Treatment of CDAD? How Should Recurrent Clostridium difficile Infection be Treated?}

While certain antibiotics have a propensity to cause CDI, antimicrobial therapy against $C$. difficile has been found to be successful in treating CDI in a clear majority of cases. In a Cochrane review that included 22 randomized controlled trials with 3215 participants, four RCTs directly compared vancomycin and metronidazole for the symptomatic cure of CDI. ${ }^{246,247-250}$ It was found that vancomycin was modestly superior to metronidazole for the treatment of $\mathrm{CDI}$ with a moderate quality of evidence. However, metronidazole has a much lower cost and an acceptable efficacy for this indication. Fidaxomicin (a newer oral antibiotic with minimal absorption) was non-inferior to vancomycin for treatment of CDI in a multicenter randomized trial. ${ }^{251}$ It was more effective than vancomycin in achieving clinical cure when patients were receiving concomitant antibiotics for concurrent infections. ${ }^{252}$ There are no direct comparisons between fidaxomicin and metronidazole, however, a network meta-analysis including studies that compared fidaxomicin with vancomycin and vancomycin with metronidazole concluded that fidaxomicin was superior to the other two agents for the sustained cure of CDI. ${ }^{253}$ Clinical cure rate following oral teicoplanin for management of CDI was comparable with oral vancomycin for management of CDI $(96.2 \%$ vs. $100 \%$, $\mathrm{p}=0.56) .{ }^{254}$ Similar cure rates were reported on comparing teicoplanin with both metronidazole and vancomycin for management of CDI. ${ }^{255}$ A pertinent question is whether the offending antibiotic should be stopped during treatment of $C$. difficile infection. A retrospective review of 246 patients found that the use of implicated antibiotics after the completion of CDI treatment was significantly associated with recurrence of CDI compared to no antimicrobial use [odds ratio 
(OR) 3.02; 95\% CI, 1.66-5.52]. On the contrary, the use of the implicated antibiotic during the CDI therapy was not associated with recurrent CDI (OR 0.79; 95\% CI, $0.40-1.52){ }^{256}$ This suggests that treatment of the primary infection may continue, if necessary, with appropriate antibiotic under the cover of CDI therapy.

Use of probiotics in addition to antibiotics for treatment of CDI showed that probiotics reduced the rate of recurrence in patients with recurrent CDI but not in patients with an initial episode. ${ }^{257}$ In a systematic review use of probiotics in the treatment of CDI was not effective. ${ }^{258}$ Whilst probiotics are unsuccessful in the treatment of CDI, they have been found to be beneficial for preventing CDI in patients receiving antibiotics. In a review of 26 RCTs, probiotics (including Lactobacillus, Saccharomyces, and combinations) significantly reduced the risk of developing CDAD by $60.5 \%(R R=0.395 ; 95 \%$ CI 0.294-0.531; $\mathrm{p}<0.001){ }^{259}$

Recurrent CDI occurs in up to one-third of the patients and is associated with considerable morbidity and costs. A systemic review that included three studies comparing vancomycin with metronidazole, reported that vancomycin and metronidazole are equally effective in the treatment of recurrent CDI. ${ }^{260-263}$ Addition of Saccharomyces boulardii to vancomycin significantly decreased the recurrence rate $(16.7 \%$ vs. $50 \%, \mathrm{p}=0.05) .{ }^{263}$ Fidaxomicin was more effective as compared to vancomycin for recurrent CDI (RR 1.86, 95\% CI 1.04-3.31, p = 0.04). ${ }^{251,264}$ Fecal microbiota transplantation has also been compared to drug therapy for treatment of recurrent CDI. It was found that vancomycin therapy with a duodenal infusion of donor feces had relapse free cure rate of $93.8 \%$ as compared to $30.8 \%$ and $23.1 \%$ in vancomycin with bowel lavage and vancomycin therapy alone respectively. ${ }^{265}$

\section{Evidence Statement}

Both metronidazole and oral vancomycin have similar efficacy in the clinical and bacteriologic cure of CDI. Use of implicated antibiotic after completing the treatment of CDI is associated with increased risk of recurrence of CDI. There is insufficient evidence to justify the use of probiotics as an adjunct to antibiotics in the treatment of CDAD. In a single RCT, fecal microbiota transplantation was found to be highly efficacious for treatment of recurrent CDI.

\section{Recommendations}

- We recommend metronidazole as the first line treatment of mild to moderate CDI/CDAD (1A).

- We recommend oral vancomycin as the first line treatment of microbiologically proven severe CDI/ CDAD (1A).
- We recommend oral vancomycin as the treatment of recurrent CDI/CDAD infection (2A).

- We recommend fecal microbiota transplantation as an alternate treatment of recurrent CDI/CDAD infection (2A).

- We recommend that implicated antibiotics should be discontinued as soon as clinically feasible (2A).

- We recommend against the use of probiotics as an adjunct for the treatment of CDI/CDAD (2A).

- We recommend the addition of vancomycin to a patient with microbiologically proven CDI/CDAD if the patient is already on metronidazole or has no clinical response to metronidazole within 3 to 4 days. (UPP)

\section{ABDOMINAL INFECTIONS IN ICU}

\section{Acute Pancreatitis and Infected Pancreatic Necrosis}

Acute pancreatitis (AP) is the inflammatory condition of the pancreas characterized clinically by abdominal pain and raised serum levels of pancreatic enzymes. ${ }^{266}$ Majority of the cases are caused by cholelithiasis and chronic alcohol consumption. ${ }^{267,268}$ Depending on the severity, AP is divided into mild, moderate and severe. Severity of pancreatitis is based upon the presence of organ failure and complications of acute pancreatitis either local or systemic. ${ }^{269}$ Local complications include peripancreatic fluid collections and pancreatic or peripancreatic necrosis (sterile or infected) whereas systemic complications include failure of an organ system (respiratory, cardiovascular, or renal) and exacerbation of a pre-existing disorder (e.g., chronic obstructive pulmonary disease, heart failure, or chronic liver disease). ${ }^{270}$ Patients with mild AP have no evidence of organ failure, local or systemic complications. Moderately severe AP is defined by the presence of transient organ failure lasting less than 48 hours with or without local and systemic complications. Persistent organ failure for more than 48 hours associated with local and systemic complications defines severe AP (SAP). ${ }^{269,271}$ About 20 to $30 \%$ of patients with AP develop acute necrotizing pancreatitis. ${ }^{272,273}$ Pancreatic necrotic tissue may remain sterile $(\sim 70 \%)$ or may get infected $(\sim 30 \%)$. The severity of necrotizing pancreatitis is determined on the basis of the extent of parenchymal involvement by necrosis (i.e., $<30 \%, 30-50 \%$, and $>50 \%) .{ }^{274}$ Infected pancreatic necrosis is associated with higher mortality as compared to sterile necrosis. ${ }^{275,276}$ Thus, early recognition and the institution of appropriate therapy is necessary. Treatment options include administration of antibiotics and surgical intervention if there is no response to antibiotics. ${ }^{277,278}$ 


\section{What are the Incidence, Risk Factors, and Microbiology of Pancreatic Infection Following Acute Pancreatitis?}

\section{Incidence and Risk Factors for Infected Pancreatic Necrosis}

The incidence of infected pancreatic necrosis (IPN) in patients with acute pancreatitis varies from 12 to 37\% depending upon the patients included (AP vs. SAP) and diagnostic modality used for IPN. ${ }^{279-282}$ Patients with necrotizing pancreatitis are more prone to develop a pancreatic infection and organ failure. ${ }^{275,276}$ Greater the extent of necrosis more likelihood of IPN. In a retrospective review of 300 patients of $\mathrm{AP}$, pancreatic infection and organ failure were directly related to the extent of pancreatic necrosis. ${ }^{281}$ In a prospective single-center study that included 204 patients of AP, pancreatic necrosis of more than $50 \%$ was significantly associated with the development of pancreatic infection and multiorgan failure. ${ }^{282}$ In a prospective observational study from India, similar findings were reported. ${ }^{279}$ Patients of AP can develop organ failure either during the early phase $(<1$ week) known as primary organ failure or during a later phase of AP (>1 week) known as secondary organ failure. ${ }^{283,284}$ In a prospective observational study in 805 patients of acute pancreatitis, the presence of primary organ failure was associated with mortality of $15.8 \%$ and was a risk factor for the development of infected pancreatic necrosis in $76 \%$ of patients. $^{285}$

\section{Evidence Statement}

The incidence of pancreatic infection following acute pancreatitis ranges from 12 to $37 \%$. Presence of pancreatic necrosis of $>50 \%$ is a major risk factor for pancreatic infection following acute pancreatitis. Primary organ failure predicts the development of infective pancreatic infection in patients with acute pancreatitis.

\section{Microbiology of Pancreatic Infection Following Acute Pancreatitis}

Enteric gram-negative bacteria including E. coli, Klebsiella, Pseudomonas, and Enterobacteriaceae are the most common organisms isolated from IPN. ${ }^{286,279,287}$ It has been demonstrated that translocation of enteric bacteria (from the gut) is the main source of infection in necrotizing pancreatitis. ${ }^{288,289}$ A recent prospective observational study from India evaluated 209 patients with AP; 108 (52\%) developed infected pancreatic necrosis (IPN). Polymicrobial infection was seen in $51 \%$ patients. Most common GNB isolated was E. coli (32\%), E. faecium was the most common gram-positive organism (7\%), whereas fungi were isolated in $13 \%$ cases. Importantly, $42 \%$ of isolates were MDR, whereas $25 \%$ were XDR. ${ }^{290}$ If required, was delayed beyond 4 weeks and done primarily employing minimally invasive techniques. The primary outcome measure was independent predictors of in-hospital mortality. Of 209 patients with AP, 81 (39\%) gram-positive bacteria including Staphylococcus aureus, Streptococcus faecalis, Enterococcus as well as anaerobes, and fungi have also been found. ${ }^{291,292}$ There are several studies that reported increase in the incidence of IPN caused by gram-positive organisms especially in patients who received prophylactic antibiotics for the prevention of development of IPN. ${ }^{282,293-295}$ Garg et al reported that the majority of the isolates from IPN were sensitive to third-generation cephalosporins and quinolones. A more recent study from India observed that amikacin and imipenem were active against the majority of the gram-negative organisms isolated from IPN. ${ }^{279,287}$ Resistance in gram-negative organisms to aminoglycosides, quinolones, beta-lactam / beta-lactamase inhibitors as well as to carbapenems has increased over last few decades. However, they remain sensitive to colistin and tigecycline. Gram-positive organisms remained sensitive to vancomycin, linezolid, and teicoplanin.

\section{Evidence Statement}

Gram-negative organisms are the most common organisms isolated from infected pancreatic necrosis following acute pancreatitis in Indian patients. Prophylactic antibiotic use in patients of AP to prevent IPN has been associated with increased risk of infection with gram-positive organisms. Resistance to carbapenems, beta-lactam/betalactamase inhibitors and quinolones in gram-negative organisms isolated from IPN has increased, however, with maintaining sensitivity to colistin and tigecycline.

\section{What are the Empirical Antibiotics of Choice for Treatment of Pancreatic Infection Following Acute Pancreatitis?}

Initial reports on use of prophylactic antibiotics in patients with AP to prevent IPN was associated with a reduction in the incidence of IPN and mortality, however, well-designed RCTs and meta-analysis failed to confirm the advantage of prophylactic antibiotics. ${ }^{280,296,297}$ Antibiotics should be prescribed in patients with evidence of IPN (positive image-guided FNA or surgical specimen) or suggested by presence of air within the necrotic pancreatic tissue or persistent fever with leucocytosis and multiorgan failure. ${ }^{277,278}$ Empirical antibiotic regimen is selected based upon the local susceptibility pattern, pharmacokinetic properties of antibiotics and previous antibiotic exposure. Gramnegative organisms isolated from IPN show varying susceptibility to aminoglycosides, cephalosporins, quinolones, piperacillin-tazobactam, and carbapenems. 
Over the past few decades, there is an increase in the resistance among GNBs isolated from IPN to cephalosporins, quinolones, piperacillin-tazobactam and carbapenems with maintained sensitivity to colistin. Various pharmacokinetic studies have demonstrated the existence of blood pancreatic barrier and this barrier is responsible for the selective uptake of antibiotic drugs into the pancreas. ${ }^{298,299}$ These studies demonstrate that carbapenems have the highest while as aminoglycosides have the least penetration to pancreatic tissue. ${ }^{299}$

Duration of antibiotic therapy in patients with IPN is not clear. However, Malaysian society of intensive care suggests that duration should be guided by a serial assessment of clinical and radiological response. ${ }^{300}$ There are multiple case series, observational studies, and meta-analysis which suggest that conservative management with the use of antibiotics in patients with IPN is associated with improved outcome and less mortality as compared to surgical debridement. ${ }^{301-305}$ Percutaneous drainage or endoscopic necrosectomy should be considered if the patient fails to improve or deteriorates clinically. ${ }^{277,278}$

\section{Evidence Statement}

Prophylactic use of antibiotics in patients with necrotizing pancreatitis has not been shown to reduce the incidence of pancreatic infection and mortality. Presence of persistent fever, leucocytosis, multiorgan failure and presence of air within pancreatic necrosis suggest infected pancreatic necrosis. Cephalosporins, piperacillin-tazobactam, quinolones, and carbapenems have the highest whereas aminoglycosides have the lowest penetration into necrotic pancreatic tissue. Response to antibiotic therapy is assessed by clinical and radiological parameters.

\section{Recommendations}

- Routine use of prophylactic antibiotics to prevent pancreatic infection following acute pancreatitis of any severity is not recommended (1A).

- Empirical antibiotic regimen in patients with infected pancreatic necrosis should be guided by local microbiological data, susceptibility pattern, the pharmacokinetic property of antibiotics and previous antibiotic exposure (UPP).

- In treatment-naïve patients with evidence of infected pancreatic necrosis, we recommend empirical treatment with either carbapenems, piperacillin-tazobactam or cefoperazone- sulbactam (2A).

- In patients not responding or already exposed to the piperacillin-tazobactam, cefoperazone- sulbactam or carbapenems, colistin should be added to the empirical regime $(3 \mathrm{~B})$.
- Duration of antibiotic therapy should be guided by clinical, radiological and laboratory parameters (UPP).

- Patients not responding to antibiotics should undergo necrosectomy and drainage (3B).

\section{BILIARY SEPSIS}

\section{Acute Cholangitis}

Acute cholangitis (AC) is a bacterial infection of the biliary tract that commonly occurs in an obstructed system and leads to systemic signs of infection. Choledocholithiasis is the leading cause of acute cholangitis. ${ }^{306} \mathrm{AC}$ is classified as mild, moderate and severe based on organ dysfunction and various biochemical abnormalities. ${ }^{307}$ Grade III AC is associated with organ dysfunction that includes any of the following: hypotension requiring either inotropic or vasopressors, confusion, Pao2:Fio2 ratio $<300$, serum creatinine levels $>$ $2 \mathrm{mg} / \mathrm{dl}$, an international normalized ratio $>1.5$ or platelet counts $<100 \times 10^{9} / \mathrm{L}$. Grade II cholangitis is associated with any two of the following conditions: WBC count $>12,000$ / $\mathrm{mm}^{3}$ or $<4,000 / \mathrm{mm}^{3}$, high fever $\left(\geq 39^{\circ} \mathrm{C}\right)$, age $>75$ years, hyperbilirubinemia ( $>5 \mathrm{mg} / \mathrm{dL}$ ) or hypoalbuminemia. Grade I do not meet any of the grade III or grade II criteria. Management of acute cholangitis depends on the severity of the illness and include administration of antibiotics and biliary drainage to relieve the obstruction. Drainage can be done selectively in patients with mild cholangitis, within 24-48 hours in patients with moderate cholangitis and immediately in case of severe cholangitis. ${ }^{308}$

\section{What is the Incidence, Risk Factors and Microbiology of Biliary Infection in ICU? What are the Empirical Antibiotics of Choice for Treatment of Biliary Infections in ICU?}

\section{Incidence and Risk Factors}

The incidence of acute cholangitis varies with underlying etiology. In patients with cholelithiasis symptomatic acute cholangitis develops in 0.2 to $9 \%$ of cases. ${ }^{309,310}$ The incidence of acute cholangitis after endoscopic retrograde cholangiopancreatography (ERCP) ranges from 0.4 to $10 \%{ }^{311,312}$ Risk factors for acute cholangitis include obstruction of the biliary tree (choledocholithiasis, biliary stricture, cholangiocarcinoma, periampullary carcinoma, stent placement for biliary drainage or worm infestation) or biliary intervention (ERCP, post-surgical biliary stricture). ${ }^{313-317}$

\section{Evidence Statement}

Incidence of acute cholangitis varies with underlying etiology and ranges from 0.2 to $10 \%$. Cholelithiasis, choledocholithiasis, benign and malignant common bile duct (CBD) strictures, CBD interventions and stenting are the most common risk factors for cholangitis. 


\section{Microbiology of Acute Cholangitis}

Various observational studies among patients with acute cholangitis from India and across the world have reported that gram-negative enteric organisms are the most common pathogens isolated from bile and/or blood. ${ }^{315,318-322}$ In patients with nosocomial acute cholangitis e.g., postoperative state, with indwelling biliary stents or those with malignant biliary obstruction, more resistant organisms such as MRSA, VRE, and Pseudomonas are frequently detected as causative microorganisms. Risk factors for MDR organisms causing acute cholangitis include previous hospitalization and antibiotic use within 90 days. ${ }^{317}$ Although the bacteriological profile of acute cholangitis has remained stable over the last few decades, their antibiotic susceptibility pattern has changed. Most of the gram-negative isolates show varying sensitivity to carbapenems, piperacillin-tazobactam, cefoperazone-sulbactam, aminoglycosides and quinolones, with increased resistance to cephalosporins and penicillins. ${ }^{315,317,319-322}$

\section{Evidence Statement}

Gram-negative organisms are the most common organisms isolated from patients with acute cholangitis. Most of the pathogens isolated are susceptible to third generation cephalosporins (such as cefoperazone-sulbactam), aminoglycosides, quinolones, ureidopenicillins and carbapenems. Risk factors for multidrug drug resistance organisms causing acute cholangitis include indwelling biliary stent, malignant biliary obstruction, previous hospitalization and antibiotic use within 90 days.

\section{What is the Empirical Antibiotic Regimen for Acute Cholangitis?}

Empirical antibiotic regimen in patients with acute cholangitis depends on the antimicrobial activity against causative bacteria, severity of cholangitis, past history of antimicrobial administration to the patient, local susceptibility patterns (antibiogram) of the suspected causative organisms and biliary penetration of the antimicrobial agents. ${ }^{323}$ Biliary obstruction reduces the antibiotic concentration within the bile and improves after biliary drainage, therefore should be considered in all patients of acute cholangitis. ${ }^{308}$ Tokyo guidelines for management of acute cholangitis suggest monotherapy with beta-lactam/ beta lactamase inhibitor (cefoperazone-sulbactam, piperacillin-tazobactam) or carbapenems or fluoroquinolone plus metronidazole to cover anaerobes. ${ }^{324}$ IDSA suggests combination of beta-lactam/ beta lactamase inhibitor (BL/BLI) or carbapenems or quinolones with metronidazole for moderate to severe community acquired cholangitis.
For nosocomial moderate to severe cholangitis combination of BL/BLIs or carbapenems or quinolones with metronidazole plus vancomycin is advised. ${ }^{325}$ IDSA suggests that antimicrobial therapy of established infection should be limited to 4-7 days, unless it is difficult to achieve adequate source control. ${ }^{325}$ Previous Tokyo guidelines recommended antibiotics for 2-3 days in case of mild and 5-7 days in case of moderate to severe cholangitis. ${ }^{324}$ However, latest revised Tokyo guidelines for management of acute cholangitis suggest duration of antibiotic to be 4-7 days once the source of infection is controlled. ${ }^{323}$ Duration of antibiotics may be guided by clinical response.

\section{Evidence Statement}

Empirical antibiotic regime in patients with acute cholangitis is guided by the severity of the disease, local antibiotic susceptibility pattern and biliary penetration of the antibiotics. Duration of antibiotics depends on the severity of cholangitis and adequacy of source control. Biliary drainage (percutaneous or endoscopic) is required in addition to antibiotic use in management of acute cholangitis.

\section{Recommendations}

- Empirical antibiotic therapy should be guided by severity of the cholangitis, local microbiological susceptibility patterns, biliary penetration of antibiotics and previous antibiotic exposure (UPP).

- We recommend either beta-lactam/beta-lactamase inhibitor (such as cefoperazone-sulbactam or piperacillin/tazobactam) or carbapenems (imipenem/ meropenem) as monotherapy in patients with moderate to severe cholangitis (3B).

- We recommend antibiotic duration for 4-7 days in patients of acute cholangitis after adequate source control (2B).

- Biliary drainage should be considered in all patients with cholangitis in addition to empirical antibiotic therapy. (1A)

\section{Liver Abscess}

Liver abscess is an infectious, space-occupying lesion in the liver. Pyogenic and amoebic liver abscess are the two most common causes of liver abscess. Appropriate initiation of antibiotics will help to prevent potentially lethal complications like bacteremia and spread of abscess to other organs.

\section{Incidence and Risk Factors}

The incidence of pyogenic liver abscess varies from as low as 2.3 per lac population to as high as 446 per lac depending upon the presence of risk factors which predispose the 
person to liver abscess. ${ }^{326,327}$ The various risk factors for pyogenic liver abscess include male gender, older age, diabetes mellitus, biliary diseases, endobiliary procedures, alcoholism, hepatobiliary malignancies, and infected cystic liver lesions. ${ }^{327-331}$ and early diagnosis may be difficult.

\section{What are the Most Common Organisms Causing Liver Abscess in ICU?}

Microorganisms causing liver abscess have shown varying trends over the years. The earlier studies had shown predominantly gram-positive organisms like Streptococcus as common cause of pyogenic liver abscess. ${ }^{332}$ However, the recent studies have reported gram-negative organisms (including klebsiella pneumoniae, E. coli, P. aeruginosa) to be responsible for majority of cases of pyogenic liver abscess. ${ }^{328,329,333-337}$ Rarely pyogenic liver abscess is caused by organisms like burkholderia, prevotella and anaerobic bacteria including eikenella and Peptostreptococcus. ${ }^{338,339}$ In Indian setting, amoebic liver abscess is the most common cause of liver abscess caused by infection with Entamoeba histolytica. ${ }^{340}$

\section{Evidence Statement}

Amoebic liver abscess is the most common cause of liver abscess in Indian setup. The incidence of pyogenic liver abscess varies from 2.3 to 446 per 100000 hospital admissions per year. Gram-negative organisms (E. coli and klebsiella) are the most common organisms causing pyogenic liver abscess. Risk factors for pyogenic liver abscess include diabetes mellitus, older age, male gender, biliary diseases, biliary procedures, alcoholism, malignancy, intra-abdominal infection, and cystic lesions in the liver.

\section{What are the Empirical Antibiotics of Choice for Treating Liver Abscess in ICU?}

\section{Amoebic Liver Abscess}

Empirical treatment of amoebic liver abscess consists of a combination of tissue agent and a luminal agent. Metronidazole is the drug of choice for management of amoebic liver abscess. Metronidazole given for a period of 10 days has been shown to be effective. ${ }^{341}$ Alternatives to metronidazole include tinidazole, ornidazole, and nitazoxanide. ${ }^{342,343}$ The luminal agents used to remove any intraluminal cysts include paromomycin, diiodohydroxyquin or diloxanide, even if the stool microscopy is negative. Routine use of drainage of amoebic liver abscess is not indicated in uncomplicated cases. ${ }^{341}$ However, addition of needle aspiration to metronidazole has shown to hasten clinical improvement especially in large abscess $(5 \mathrm{~cm}$ to $10 \mathrm{~cm}){ }^{337}$ Surgical intervention is required ifthere is noresponse to medical management. ${ }^{341,344}$

\section{Evidence Statement}

Metronidazole is the drug of choice for treatment of amoebic liver abscess. The optimum duration of treatment in patients with amoebic liver abscess is 10-14 days. Routine needle aspiration of amoebic liver abscess is controversial. Addition of aspiration to drug therapy in patients with amoebic liver abscess of $>5 \mathrm{~cm}$ in size hastens clinical improvement.

\section{Recommendations}

- We recommend metronidazole as an initial antibiotic of choice in patients with amoebic liver abscess (2A).

- We recommend antibiotic treatment for a period of 10-14 days in patients with amoebic liver abscess (3B).

- Needle aspiration of amoebic liver abscess is recommended in patients with lack of clinical improvement in 48-72 hours, left lobe abscess, abscess more than $5-10 \mathrm{~cm}$ or thin rim of liver tissue around the abscess $(<10 \mathrm{~mm})$ (UPP).

\section{Pyogenic Liver Abscess}

Antibiotics that are effective in treatment of pyogenic liver abscess include third and fourth generation cephalosporins (such as ceftriaxone, cefepime), aminoglycosides, fluoroquinolones, beta-lactam/ beta-lactamase inhibitor (piperacillin-tazobactam), carbapenems, and metronidazole. ${ }^{334,345-347}$ Carbapenems are effective for treatment of liver abscess caused by melioidosis or infection with ESBL producing organism. ${ }^{348,349}$

Empirical regimen should include a broad-spectrum parenteral antibiotic pending microbiologic analysis of the abscess contents. It should cover enteric gram-negative bacilli, streptococci, and anaerobes. Antibiotic therapy should generally be continued for four to six weeks. 335 However, the optimal duration of therapy is unclear and is guided by clinical and radiological response. Studies have reported that shorter courses of antibiotics for 2-4 weeks are effective as well. ${ }^{335,345,350}$ In case of abscess cavity with a size less than $5 \mathrm{~cm}$, a needle aspiration is preferred and in case of abscesses more than $5 \mathrm{~cm}$ in size, percutaneous catheter drainage is preferred. ${ }^{351-353}$ Surgical drainage is required in cases of abscesses with viscous contents obstructing the catheter, underlying disease requiring primary surgical management and inadequate response to percutaneous drainage within 7 days. ${ }^{354}$

\section{Evidence Statement}

Beta-lactam/beta-lactamase inhibitors, metronidazole and carbapenems are effective antibiotics for management of pyogenic liver abscess. Carbapenems are effective in case 
of suspected infection with ESBL producing organisms or melioidosis. Antibiotics are required for prolonged periods ranging from 2-4 weeks. Clinical and radiological assessment is required to guide the adequate treatment duration.

\section{Recommendations}

- We recommend beta lactam/beta lactamase inhibitors with metronidazole in patients with pyogenic liver abscess for a duration of 2 to 4 weeks (2A).

- We recommend carbapenems in case of infection with ESBL producing organisms or melioidosis (2B).

\section{Peritonitis}

Peritonitis is defined as an inflammation of the peritoneum from any cause. Peritonitis occurs due to a variety of etiologies, of which the most common is infections. It is broadly classified as primary, secondary and tertiary.Primary peritonitis, also known as spontaneous bacterial peritonitis (SBP), has no identifiable anatomical dehiscence. It is usually managed non-surgically. The risk factors for development of primary peritonitis include advanced cirrhosis, nephrotic syndrome and peritoneal dialysis. ${ }^{355,356}$ Secondary peritonitis is the infection of peritoneum that occurs in critical ill patients secondary to organ perforation, anastomotic leak or trauma to the gastrointestinal tract. Tertiary peritonitis may be defined as a severe recurrent or persistent intra-abdominal infection after apparently successful and adequate surgical source control of secondary peritonitis. ${ }^{357} \mathrm{It}$ leads to prolonged systemic inflammation and is usually associated with high mortality (30-64\%). Longer ICU stay, emergency abdominal surgery and total parenteral nutrition are risk factors associated with the development of tertiary peritonitis. ${ }^{358-363}$

\section{What are the Most Common Organisms Causing Peritonitis in ICU?}

Enteric gram-negative organisms including E. coli, klebsiella and Enterobacteriaceae are the most common causative agents for primary and secondary peritonitis. ${ }^{364,365}$ Other organisms include gram-positive bacteria (such as Enterococcus) as well as anaerobes (i.e. Bacteroides). ${ }^{365}$ Tertiary peritonitis is usually due to opportunistic and nosocomial drug resistant bacteria and fungi. Various organisms reported are Enterococcus, Candida, Staphylococcus and enterobacter. ${ }^{363,366}$

\section{Evidence Statement}

The risk factors for development of primary peritonitis are decompensated cirrhosis, nephrotic syndrome and peritoneal dialysis. The risk factors for development of secondary peritonitis include intra-abdominal organ perforation, post intra-abdominal surgery, and trauma. Longer ICU stay, urgent operation on hospital admission, total parenteral nutrition and stomachduodenum as primary infection site are associated with the development of tertiary peritonitis. Gam-negative enteric organisms are the common causes of primary and secondary peritonitis. Other organisms include grampositive as well as anaerobic bacteria. The organisms commonly isolated in tertiary peritonitis are Candida, Enterococcus faecium and Staphylococcus epidermidis.

\section{What are the Empirical Antibiotics of Choice for Treating Peritonitis in ICU? \\ Primary Peritonitis}

Cephalosporins and fluoroquinolones are effective against majority of the cases of primary peritonitis. ${ }^{364,367-370}$ Antibiotics for a period of 7-10 days are effective in SBP. ${ }^{364,367}$ In difficult to treat SBP, cefepime and imipenem are reported to be effective. ${ }^{371}$

\section{Secondary Peritonitis}

The antibiotics effective in secondary peritonitis are beta lactam/beta lactamase inhibitors (piperacillintazobactam), quinolones, carbapenems, aminoglycosides and metronidazole. ${ }^{365,372,373}$ When enterococci are considered, addition of vancomycin or linezolid is required for a spectrum adequacy rate of more than $95 \% .{ }^{374}$ The average duration of antibiotic therapy is 10 to 14 days. However, recently the emphasis is on a shorter course of antibiotics after adequate source control.The recent STOP-IT trial has found that in patients after an adequate source control, outcomes after fixed-duration antibiotic (approximately 4 days) were similar to those after a longer course of antibiotics (approximately 8 days). ${ }^{375}$

\section{Evidence Statement}

Third generation cephalosporins are the most effective antibiotic therapy for primary peritonitis. Antibiotics are usually required for 7-10 days for adequate treatment. Most of the organisms isolated in secondary peritonitis are sensitive to beta lactam/beta lactamase inhibitors or carbapenems. For gram-positive organisms, vancomycin and linezolid are effective treatment options. Short duration of antibiotic treatment (4 days) are as effective as longer duration after an adequate source control.

\section{Recommendations}

- We recommend third generation cephalosporins (such as cefotaxime and ceftriaxone) for a duration of 7-10 days in patients with primary peritonitis (2A). 
- We recommend either beta-lactam/beta-lactamase inhibitor or carbapenems with an anaerobic cover (using metronidazole) for the treatment of secondary peritonitis (2A).

- For secondary peritonitis antibiotic treatment is required for 4 days after an adequate source control(2A).

\section{CNS Infections in ICU}

Infections of central nervous system (CNS), either community or hospital acquired, are frequent causes of admission to ICU. Bacterial meningitis and brain abscess are one of the commonest CNS infections and can result in significant morbidity and mortality. CNS infections are markedly different from systemic infections because of closed anatomic space and immunologic isolation of CNS from the rest of the body.They often have nonspecific clinical manifestations posing a diagnostic challenge to the clinician. Early suspicion, rapid diagnosis and aggressive management are essential for better outcomes and to prevent various complications and neurological sequalae.

\section{What are the Most Common Organisms Causing Acute Bacterial Meningitis in ICU?}

Bacterial meningitis, an infection of meninges and subarachnoid space, is a complex disorder in which injury is caused partly by the causative organism and partly by the host inflammatory response. Bacterial meningitis is a medical emergency, given the associated mortality and neurological sequalae requiring prompt recognition, rapid diagnostic evaluation and emergent antimicrobial therapy. Hence accurate information regarding incidence, risk factors and microbiological profile of bacterial meningitis is necessary to ensure appropriate empirical antibiotic management. Meningitis can be community acquired or associated with a variety of neurosurgical procedures (e.g., craniotomy, placement of invasive neuro-monitoring techniques, external ventricular drain catheters or cerebrospinal fluid shunts) and penetrating head injury. The latter group is classified as nosocomial meningitis or healthcare associated meningitis and ventriculitis. Both groups differ in their pathogenic mechanisms, risk factors, etiological agents microbial susceptibility patterns and hence are discussed separately.

\section{Community Acquired Meningitis}

The incidence of bacterial meningitis in USA was 2 cases per 100,000 population in 1998-1999 that decreased to 1.38 cases per 100,000 population in 2006-2007; most common organisms were Streptococcus pneumoniae $(56.8 \%)$, neisseria meningitidis (17.2\%), group B streptococci
(16.7\%), Hemophilus influenzae (5.8\%) and listeria monocytogenes $(3.2 \%){ }^{376}$ In a retrospective study of 195 culture positive acute bacterial meningitis patients, most common organism was Streptococcus pneumoniae followed by Staphylococcus aureus and klebsiella pneumoniae. ${ }^{377}$ Various large studies have found S. pneumoniae as the most common etiological agent followed by $N$. meningitidis, L. monocytogenes, $H$. influenzae and group B Streptococcus, ${ }^{378-382}$ though S. aureus has also been reported as one of the commonetiological agents by some. ${ }^{379,380}$ Otitis media, immunocompromised status, elderly population and prior use of antibiotics have been described as risk factors for bacterial meningitis. ${ }^{378,383,384}$ Various Indian studies have yielded similar results. ${ }^{385-388}$

\section{Evidence Statement}

The incidence of community acquired pyogenic meningitis ranges from 2 to 7.40 per lakh population. The common causative organisms include Streptococcus pneumoniae, neisseria meningitidis, other streptococci, Hemophilus influenzae and listeria monocytogenes. Other causative organisms are Staphylococcus species, gram negative bacilli, Pseudomonas and Acinetobacter. Common risk factors for community acquired bacterial meningitis are otitis media, elderly population, depressed immune status and prior use of antibiotics.

\section{Nosocomial Meningitis}

Nosocomial meningitis may result from various invasive procedures including craniotomy, placement of internal or external ventricular catheters, lumbar puncture, intrathecal infusions of medications, spinal anesthesia or complicated head trauma or rarely from metastatic infection in patients with hospital-acquired bacteremia.

Incidence of post ventricular drain or catheter related infections has been studied in many retrospective and prospective studies and ranges from 5.6 to $14.2 \%$ and 5.5 to $19 \%$ respectively. ${ }^{389-394}$ A systematic review from January 1990 through March 2008 reporting on ventriculostomy and extra-ventricular drain (EVD) related CNS infections described incidence of $2-27 \%{ }^{395}$ Staphylococcus epidermidis (70\%) is the most common microbiological agent followed by gram negative bacilli $(15 \%)$ and Staphylococcus aureus (10\%).Risk factors described included EVD duration greater than 11 days, frequency of cerebrospinal fluid (CSF) sampling, intraventricular hemorrhage and surgical technique (subcutaneously tunneled EVD, Rickham reservoir with percutaneous CSF drainage). Post craniotomy or neurosurgery incidence of meningitis ranges from 0.02 to $9.5 \% .{ }^{391,396-402}$ Most of the studies 
have reported Staphylococcus to be the most common causative organism. ${ }^{391,396,398,400,401}$ Few studies have also reported Acinetobacter and Enterobacteriaceae as the most common organisms. ${ }^{397,399}$ Postoperative CSF leak has been consistently shown to be a risk factor. ${ }^{391,396-398,400,401,403}$ Other risk factors are placement of external shunts, longer duration of drainage, multiple intracranial operations, emergency or prolonged surgery, diabetes and elderly population..$^{391,396-401}$ The role of prophylactic antibiotics for post neurosurgery and craniotomy meningitis had been debatable, however, a recent meta-analysis of 7 RCTs including 2365 postcraniotomy patients found that prophylactic antibiotic use reduced the rate of post neurosurgical meningitis. ${ }^{404}$ The incidence of post spinal blockade meningitis is very low with a large retrospective analysis of 12,60,000 spinal blockades and 450,000 epidural blockades showing incidence to be 1 in 53000 with alpha-hemolytic streptococci as the most common causative organism. ${ }^{405}$ Exogenous inoculation is a risk factor and various measures such as hand disinfection, sterile gloves, face masks and operating caps decrease the risk of development of meningitis. ${ }^{406}$ The incidence of meningitis or ventriculitis in patients with post traumatic head injury is $1.39-2 \% .407,408$ Common organisms include CONS, gram negative bacilli and Acinetobacter. Lumbar and ventricular drains are described as the risk factors. A recent Cochrane systematic review has not shown benefit of using prophylactic antibiotics in patients with basilar skull fracture, independent of CSF leakage. ${ }^{409}$ Post internal ventricular drain infections incidence has been reported between 5.9 to $15.2 \%$ in various prospective and retrospective studies. Most common causative organisms included Staphylococcus aureus and CONS. ${ }^{410,411}$ Postoperative CSF leak, use of single gloves and number of times shunt system exposed to breached surgical gloves were described as risk factors. ${ }^{412}$

\section{Evidence Statement}

Incidence of post ventricular drain or catheter meningitis ranges from $2 \%$ to $27 \%$.Commonly implicated organisms are CONS (especially Staphylococcus epidermidis), Staphylococcus aureus, Acinetobacter, Pseudomonas and Enterobacteriaceae. Risk factors are repeated catheterization, higher catheter duration, CSF sampling, presence of concomitant systemic infection and surgical technique i.e., subcutaneously tunnelledextraventricular drain (EVD), Rickham reservoir with percutaneous CSF drainage. Incidence of post craniotomy or post neurosurgery meningitis is 0.02 to $9.5 \%$.Most commonly implicated organisms are Staphylococcus aureus, coagulase negative staphylococci (especially S. epidermidis), Enterobacteriaceae, Acinetobacter and Pseudomonas. Risk factors include CSF leak, EVD, longer duration of drainage, multiple operations, lack of antibiotic prophylaxis and emergency surgery. Incidence of post-neuroaxial blockade meningitis is 0.2 per 10000 with viridans streptococci and Staphylococcus aureus being common organisms. Exogenous inoculation is the main risk factor. Post head trauma meningitis incidence ranges from 1.39 to $2 \%$ with CONS, Acinetobacter and Enterobacteriaceae as common microbes and prolonged hospitalization, insertion of lumbar and ventricular drain as common risk factors. Post internal ventricular drain infection incidence ranges from 5.9 to $15.2 \%$. Most common causative organisms are CONS, Staphylococcus aureus, gram negative bacilli, group D streptococci and Propionibacterium acnes. CSF leak, single gloves use and number of times shunt exposed to breached surgical gloves are the risk factors.

\section{What are the Empirical Antibiotics of Choice for Treating Acute Bacterial Meningitis in ICU? What Should be the Duration of Antibiotic Treatment?}

Early diagnosis and urgent appropriate antimicrobial therapy along with other adjunctive therapy is necessary to reduce morbidity and mortality associated with bacterial meningitis. As isolation of microorganism takes time and sometimes it may not be isolated at all, empirical anti-microbial therapy need to be based on most likely involved organism as determined by presence of risk factors for various organisms and local antibiotic susceptibility pattern.

\section{Community Acquired Meningitis}

The evidence regarding empirical antibiotic choice in acute bacterial meningitis (ABM) is limited. A retrospective study found reduced penicillin susceptibility in $23 \%$ patients with meningitis, including $16 \%$ in community acquired meningitis. Ceftriaxone combined with penicillin was found adequate in $97 \%$ cases. ${ }^{413}$ Retrospective study by Erdem et al reported inadequacy of ceftriaxone alone in treatment of pneumococcal meningitis in view of increasing penicillin resistance in pneumococci worldwide. ${ }^{414}$ A Cochrane review in 2007 comparing third generation cephalosporins (ceftriaxone or cefotaxime) with conventional antibiotics (ampicillin-chloramphenicol combination, or chloramphenicol alone) as empirical therapy for ABM in adults and children found no statistically significant difference between the groups in the risk of death, risk of deafness or risk of treatment failure although significantly decreased chances of culture 
positivity of CSF after 10 to 48 hours with the third generation cephalosporins at the cost of increased risk of diarrhea. ${ }^{415} \mathrm{~A}$ recent Indian study including 266 culture positive ABM patients (including 142 CAM patients) found that gram positive pathogens exhibited maximum sensitivity to vancomycin and linezolid whereas most gram negative pathogens were sensitive to carbapenems. ${ }^{416}$ Seven days antibiotic therapy has been recommended for $N$. meningitidis and $H$. influenzae, 10-14 days for S. pneumoniae, 14 to 21 days for $S$. agalactiae, 21 days for aerobic GNB and 21 days or more for L. monocytogenes. ${ }^{417}$

\section{Evidence Statement}

Choice of antibiotics depends on most likely causative microorganism, local antibiotics sensitivity patterns, mechanism of infection and patient's predisposing condition. Most commonly recommended empirical antibiotic regimens include third generation cephalosporin plus vancomycin, third generation cephalosporin monotherapy and penicillin monotherapy. Addition of amoxicillin, ampicillin or benzyl-penicillin has been recommended in patients older than 50 years.

\section{Recommendations}

- We recommend third generation cephalosporin (preferably ceftriaxone) plus vancomycin as empirical antibiotics of choice for community acquired meningitis (3A).

- We recommend to add ampicillin or amoxicillin if age $>50$ years $(3 \mathrm{~A})$.

- If beta-lactams are contraindicated, we recommend chloramphenicol plus vancomycin as antibiotic of choice, and to add cotrimoxazole if age $>50$ years (3A).

- We recommend ciprofloxacin or aztreonam plus vancomycin as alternative regimen and to add cotrimoxazole, if age greater than 50 years (UPP).

- We recommend duration of antibiotics based on suspected or isolated organisms i.e., 10 to 14 days for Streptococcus pneumoniae, 14 to 21 days for Streptococcus agalactiae, 7 days for neisseria meningitidis or Hemophilus influenzae, 21 days for aerobic gram negative bacilli, and 21 days or more for listeria monocytogenes (3A).

- If no microorganism is identified, antibiotics should be given for at least 10 to 14 days (3A).

\section{Nosocomial Meningitis}

Treatment recommendations for nosocomial meningitis are largely based upon expert opinion. IDSA guidelines for management of bacterial meningitis recommend vancomycin plus third generation cephalosporin for post basilar skull fracture meningitis; vancomycin plus cefepime, ceftazidime or merepenemhas been recommended for post neurosurgery nosocomial meningitis or meningitis occurring after CSF shunt or penetrating trauma. ${ }^{417}$

A systematic review of intraventricular or intrathecal use of polymyxins in patients with gram negative meningitis including 31 case reports and case series found limited available evidence to suggest addition of intraventricular or intrathecal antimicrobials to systemic therapy in gram-negative meningitis. Toxicity was dosedependent and reversible. ${ }^{418}$ Another review for use of intraventricular use of vancomycin found its use safe and effective. ${ }^{419}$ IDSA guidelines recommend vancomycin plus an anti-pseudomonal beta-lactam (such as cefepime, ceftazidime, or meropenem) as empiric antimicrobial of choice for suspected healthcare associated ventriculitis and meningitis. ${ }^{420}$ Regarding optimum duration of antibiotic therapy, IDSA recommends therapy for 10 days if coagulase-negative Staphylococcus or P. acnes with no or minimal CSF pleocytosis, normal CSF glucose, and few clinical symptoms or systemic features; 10 to 14 day treatment is recommended in case of significant CSF pleocytosis, CSF hypoglycorrhachia, clinical symptoms or systemic features. Treatment for 21 days is recommended for gram negative bacilli and Staphylococcus aureus. In patients with repeatedly positive CSF cultures on appropriate antimicrobial therapy, IDSA recommends treatment to be continued for 10 to 14 days after the last positive culture. ${ }^{420}$

\section{Evidence Statement}

Vancomycin in combination with cefepime, ceftazidime or meropenem is commonly recommended empirical antibiotic regimen for nosocomial meningitis. Alternative regimens include third generation cephalosporin or meropenem monotherapy or ceftriaxone plus flucloxacillin or cloxacillin combination therapy. Limited available evidence shows efficacy of intraventricular or intrathecal antibiotics in management of nosocomial meningitis poorly responsive to systemic antibiotics.

\section{Recommendations}

- We recommend vancomycin plus cefepime or ceftazidime or meropenem as empirical antibiotics of choice for nosocomial meningitis (3A).

- Colistin may be given if incidence of CRE or drug resistant Acinetobacter is high in the specific unit (UPP).

- If beta-lactams are contraindicated, we recommend to replace beta-lactam with aztreonam or ciprofloxacin (3A). 
- Intraventricular or intrathecal antibiotics should be considered if infection responds poorly to appropriate systemic antibiotics clinically or microbiologically (3A).

\section{What are the Most Common Organisms Causing Brain Abscess in ICU?}

Brain abscess is a serious life-threatening emergency with high morbidity and mortality. The management of brain abscess is challenging and needs good clinical and surgical skills for better outcomes. The choice of pharmacological therapy should be based on the most likely organism, patient's predisposing condition or risk factors, mechanisms of infection, antimicrobial susceptibility patterns and on the ability of the antimicrobial agent to penetrate the abscess.

In a recent single-center retrospective study over 62 years including 620 patients of brain abscess, the incidence of brain abscess (per lakh population) was 2.5 between 1952 to $1972,2.6$ in 1980 to 1991 and 2.2 in 2002 to $2014 .{ }^{421}$ Staphylococcus aureus is one of the commonest organism followed by Proteus sp. and Streptococcus. Chronic ear infection is a common predisposing factor (65\% cases). ${ }^{422}$ Streptococcus (34\%), followed by Staphylococcus (18\%), gramnegative enteric bacilli (15\%), P. seudomonas and Haemophilus ( $2 \%$ each) were found to be the commonly isolated organisms in a recent meta-analysis. Peptostreptococcus, Bacteroides, and Fusobacterium were isolated in 3\%, $6 \%$, and $2 \%$ respectively and polymicrobial etiology was found in $23 \%$ cases. ${ }^{423}$ Most common predisposing condition was otitis media followed by sinusitis, heart disease, post-traumatic, hematogenous, pulmonary disease, postoperative, odontogenic, immunocompromised and meningitis. Two retrospective studies found Staphylococcus aureus to be the most common causative organism followed by Streptococcus. ${ }^{424,425}$ Otitis media was the most common risk factor followed by congenital heart disease, paranasal sinus infections, dental causes, trauma, and post-operative state. ${ }^{424-427}$ Various prospective Indian studies found streptococci to be most common microbe. ${ }^{426,427}$

\section{Evidence Statement}

The incidence of brain abscess ranges from 1.3 to 2.6 cases per lakh population. Most commonly involved microorganisms include Streptococcus (especially S. viridans), Staphylococcus (especially S. aureus), gram negative bacilli, anaerobes (Bacteroides, Peptostreptococcus, Fusobacterium), Pseudomonas and H. influenzae. Polymicrobial etiology accounts for $23-26 \%$ cases. Risk factors include otitis media, sinusitis, head trauma, congenital heart diseases, hematogenous spread, surgery, immunocompromised status, pulmonary disease, meningitis, and odontogenic infections.

\section{What are the Empirical Antibiotics of Choice for Treating Brain Abscess in ICU? What Should be the Duration of Antibiotic Treatment?}

The data regarding the efficacy of various empirical antibiotic regimens in the management of brain abscess is limited to observational studies and expert opinion. In a systematic review and meta-analysis of clinical characteristics and outcomes of brain abscess, 17 studies described how many patients received which regimen. ${ }^{428}$ The most common empiric treatment consisted of a third-generation cephalosporin combined with metronidazole, which was given in $53 \%$ of cases while vancomycin was added in addition $15 \%$ cases. Other regimens had combinations of chloramphenicol, metronidazole with penicillin $(9 \%)$, ampicillin, gentamicin with metronidazole ( $9 \%)$, and imipenem monotherapy $(4 \%) .{ }^{428}$ There is insufficient evidence to make specific recommendations but on the basis of limited clinical data, recommendations include cefotaxime plus metronidazole with or without rifampicin for posttrauma abscess, linezolid or vancomycin plus rifampicin plus meropenem or piperacillin/tazobactam for postsurgical abscess, cefotaxime or piperacillin-tazobactam plus metronidazole for post middle ear, paranasal sinuses, dental causes and cefotaxime with or without metronidazole or ampicillin-sulbactam for cryptogenic or metastatic abscess. Four to six weeks of antibiotic therapy is required for surgically treated abscess and 6 to 8 weeks for solely medically treated or multiple surgical abscesses with largest one treated surgically. ${ }^{429}$

\section{Evidence Statement}

The most common empiric treatment consists of a thirdgeneration cephalosporin combined with metronidazole. Antibiotic duration ranges from 4 to 8 weeks.

\section{Recommendations}

- We recommend third-generation cephalosporins plus metronidazole as the empirical antibiotic of choice for brain abscess (3A).

- We recommend adding vancomycin if there is a high likelihood of MRSA (3A).

- We recommend vancomycin plus ciprofloxacin if beta-lactams are contraindicated (3A).

- We recommend aztreonam, if ciprofloxacin cannot be given or contraindicated (UPP).

- We recommend minimum 4 weeks of therapy, however duration may be extended according to clinic-radiological response irrespective of aspiration or excision of abscess (3A). 


\section{Skin and Soft Tissue Infections in ICU}

An inflammatory microbial invasion of the epidermis, dermis and subcutaneous tissues is defined as skin and soft tissue infection (SSTI). In ICU, 4.3 to $10.5 \%$ of septic episodes may be caused by SSTIs, ${ }^{430}$ with attributable mortality of $11.7 \% .{ }^{431}$ Spectrum of SSTI includes abscess, carbuncle, cellulitis, surgical site infection, diabetic foot, and necrotizing fasciitis. SSTI has been classified based on signs of sepsis and comorbidities. SSTI without any signs or symptoms of systemic toxicity or comorbidities is termed class 1. SSTI in patients with significant comorbidities (diabetes or obesity), but without any evidence of sepsis is termed class 2. Class 3 SSTI refers to SSTI with fever, tachycardia and tachypnea with or without hypotension. Class 4 SSTI refers to life-threatening infections like necrotizing fasciitis along with sepsis. ${ }^{432}$ For treatment decision, it is important to classify SSTIs into purulent (carbuncle, furuncle, and abscess) and nonpurulent (necrotizing fascitis, cellulitis, and erysipela). Non-purulent SSTIs are classified into mild (no focus of purulence), moderate (presence of systemic inflammatory response syndrome, i.e., SIRS) and severe (failed oral antibiotics, SIRS, immunocompromised, deeper infection or organ dysfunction). Purulent SSTIs are classified into mild (no systemic signs of infection), moderate (SIRS present) and severe (SIRS along with treatment failure, or organ dysfunction). ${ }^{433}$

\section{What are the Most Common Organisms and Risk Factors for SSTI in ICU?}

Staphylococcus aureus (20.9-38.1\%) and gram-negative bacilli (29.1-57.4\%)have been commonly implicated in SSTIs in India. ${ }^{434-436}$ Pseudomonas (11.8-57.4\%) and E. coli (17.3\%) are most common GNBs. ${ }^{435,436}$ High proportion of Staphylococcus aureus (40-74\%) have been reported to be methicillin-resistant, ${ }^{435,437}$ whereas the majority of $(66.7-74 \%)$ GNBs have been reported to be ESBL producing. ${ }^{435}$ Necrotising fascitis is caused mostly by Streptococcus pyogenes in monomicrobial form. Clostridial species are also responsible for monomicrobial necrotizing fasciitis. ${ }^{438}$ In polymicrobial necrotizing fascitis, the most commonly implicated pathogens are coliforms, anaerobic bacteria and Staphylococcus. ${ }^{439,440}$ Old age, obesity, diabetes mellitus, malignancy, higher APACHE score, longer ICU stay, end-stage renal disease, cirrhosis of the liver, intravenous drug abuse, and neutropenia are risk factors for SSTI. ${ }^{432,441,442}$

\section{Evidence Statement}

Older age, diabetes mellitus, obesity, malignancy, cirrhosis, and longer ICU stay are risk factors for SSTIs.
Gram-positive organisms (Staphylococcus aureus) are the most common organism responsible for the SSTIs. E. coli and Pseudomonas are common pathogens among gram-negative organisms. MRSA and ESBL producing gram-negative organisms are the most common causative agents for SSTIs in ICU. Monomicrobial necrotizing fasciitis is commonly caused by Streptococcus pyogenes; mixed coliforms, anaerobes, and staphylococci are common causes of polymicrobial necrotizing fasciitis.

\section{What are the Empirical Antibiotics of Choice for Treating SSTI in ICU? For Empirical Therapy, Should Combination Therapy be Preferred Over Monotherapy?}

Studies on SSTIs specific to ICU settings are not available. A meta-analysis performed by Rebecca et al ${ }^{443}$ showed the clear superiority of linezolid and vancomycin in treating skin and soft tissue infection caused by S. aureus. Teicoplanin is also a good choice for treating severe SSTI caused by MRSA, with similar efficacy and fewer adverse effects as compared to vancomycin. ${ }^{44-446}$ Daptomycin has been shown to have a more rapid clinical cure, reduced the length of hospital stay and lower cost as compared to vancomycin in a prospective study of SSTIs in ICU. ${ }^{447}$ Other RCTs have demonstrated non-inferiority of daptomycin to vancomycin. ${ }^{448}$ MRSA remains sensitive to vancomycin and linezolid, and the majority remain sensitive to clindamycin also (79\%). ${ }^{437}$ For gram-negative pathogens, piperacillin-tazobactam and imipenem have been reported to be most effective antibiotics. ${ }^{430}$

\section{Evidence Statement}

Vancomycin, teicoplanin, daptomycin, and linezolid are effective in SSTIs caused by MRSA. Piperacillintazobactam and carbapenems are the most effective antibiotics for ESBL producing gram-negative organisms. Penicillin plus clindamycin are most effective antibiotics in monomicrobial necrotizing fasciitis, whereas a combination of piperacillintazobactam, fluoroquinolone and clindamycin are effective for polymicrobial necrotizing fasciitis.

\section{Recommendations}

- For moderate non-purulent SSTI, we recommend intravenous penicillin or clindamycin as the first choice of antibiotics (2A).

- Severe non-purulent SSTI should be treated with a combination of piperacillin-tazobactam along with coverage for MRSA (vancomycin, teicoplanin, daptomycin or linezolid) (2A).

- Concomitant surgical inspection or debridement should be considered for severe non-purulent SSTIs (2A). 
- For severe purulent SSTI, incision and drainage followed by empiric antibiotics including piperacillintazobactam, along with MRSA coverage (vancomycin, teicoplanin, daptomycin or linezolid) are recommended (3A).

- Penicillin plus clindamycin is recommended for monomicrobial necrotizing infection caused by Streptococcus pyogenes or clostridial species. For polymicrobial necrotizing fasciitis, a combination of piperacillin-tazobactam, fluoroquinolone and clindamycin is recommended (3A).

\section{What Should be the Duration of Antibiotic Treatment for SSTI?}

There is limited literature to guide treatment of severe or complicated SSTIs. In uncomplicated SSTI, antimicrobial administration for 5 days was equally effective to 10-day treatment. ${ }^{449}$ Complicated SSTIS may require longer treatment.

\section{Evidence Statement}

A shorter course of antibiotic therapy is adequate for uncomplicated SSTIs while complicated SSTIs require a longer duration of antibiotic therapy.

\section{Recommendations}

- Severe nonpurulent SSTIs should be treated with at least 5 days of antibiotics (3A).

- Severe SSTIs with organ dysfunction should be treated with a prolonged course of antibiotics of 2 to 3 weeks duration (3A).

\section{Sepsis of Unknown Cause in ICU}

Mortality from severe sepsis and septic shock remains consistently high. ${ }^{450,451}$ Delay in antimicrobial therapy is associated with increased in-hospital and overall mortality in severe sepsis and septic shock. ${ }^{452,453}$ Adequate source control, appropriate antibiotic therapy, and organ support are cornerstone for the success in the treatment of patients with sepsis. Delay in the initiation of appropriate antibiotic therapy has been recognized as a risk factor for mortality. While every effort should be made to secure site-specific cultures to guide microorganism-specific therapy, this should never delay the administration of empiric antimicrobials. ${ }^{454}$ Intensive efforts, including imaging, should be undertaken in an attempt to evaluate the source of infection. Two sets of blood cultures and other appropriate microbiological specimens should preferably be taken before empirical therapy. Urgent empirical broad-spectrum coverage to include all common pathogens should be administered. ${ }^{454}$

\section{What is the Empirical Treatment for Sepsis of Unknown Cause in ICU?}

There is a paucity of data on empirical antimicrobial therapy in sepsis of unknown cause in ICU. Combination antimicrobial therapy (using two drugs from a different class) improves survival and clinical outcomes in patients with sepsis who are critically ill and in septic shock as compared to monotherapy. ${ }^{455}$ Betalactams with aminoglycosides or fluoroquinolones gives a broad empirical coverage. If the patient has risk factors for MRSA, vancomycin should be added to the regimen. ${ }^{456}$ Accordingly if risk factors for MDR pathogens are present in an individual patient, betalactam of choice is a carbapenem. In India, empirical therapy should cover for various tropical infections till a definite diagnosis is reached. Third-generation cephalosporins with doxycycline is an appropriate option keeping this fact in mind.

\section{Evidence Statement}

Empirical therapy with dual class (with different mechanisms of action) combination antimicrobial therapy for sepsis of unknown cause in ICU is associated with have better clinical outcomes. Empirical therapy with either piperacillin-tazobactam or carbapenems in combination with aminoglycosideor fluoroquinolone has been shown to give appropriate broad coverage leading to better clinical outcomes as compared to monotherapy.

\section{Recommendations}

- We recommend empirical antimicrobial therapy with a combination of ceftriaxone and doxycycline or a macrolide for community-acquired sepsis of unknown origin in ICU (UPP).

- We recommend empirical antimicrobial therapy with a combination of beta-lactam/beta-lactamase inhibitor and fluoroquinolone or aminoglycoside for nosocomial sepsis of unknown origin in ICU (UPP).

- Empiric therapy should attempt to provide antimicrobial activity against the most likely pathogens based upon clinical features along with local patterns of infection and resistance (UPP).

- Duration of therapy is 7 to 10 days, though longer courses may be appropriate in patients with a slow response (3B).

\section{Empirical Antifungals for Non-neutropenic Patients in ICU}

Invasive fungal infection (IFI) is an important cause of morbidity and mortality among critically ill patients. Early institution of antifungal therapy is pivotal for mortality 
reduction. Starting targeted antifungal therapy after culture positivity or identification of pathogen requires a long time. Therefore, alternative strategies (defined as untargeted antifungal treatment) for antifungal therapy institution in patients without proven microbiological evidence of fungal infections have been considered. ${ }^{457}$ Untargeted antifungal strategies include prophylactic antifungals, pre-emptive antifungals, and empirical antifungals. Prophylaxis refers to use of antifungals without proven or suspected fungal infection but with risk factors for its development. Preemptive (diagnosis driven) approach means evidence of fungal infection, without definitive microbiological proof on the basis of surrogate biomarkers like 1-3 ß-D-glucan, mannan or anti-mannan antibodies, whereas empirical (fever-driven) approach refers to using antifungals in patients at risk for IFI, with signs and symptoms of infection, in the absence of microbiological evidence of infection. ${ }^{457}$

Among fungal pathogens, Candida spp. are the most commonly isolated microorganisms, currently being the fourth most commonly identified pathogens in nosocomial BSIs and the third most common pathogens isolated in ICU patients. ${ }^{458}$ Despite advances in antifungal therapy, the mortality associated with invasive candidiasis remains as high as $40 \% .{ }^{457}$ In India, the incidence of C. albicans ranges from 34 to $45.6 \%$ with an attributable mortality of 20 to $35.6 \%$. The incidence of non-albicans Candida is on the rise with attributable mortality ranging from 23 to $52 \%$, with higher mortality associated with Candida krusei. ${ }^{459}$ An observational study from Indian ICUs revealed an incidence of 6.5 cases per 1000 ICU admissions. There was a high prevalence of $C$. tropicalis $(41.6 \%)$, and $46.6 \%$ isolates were susceptible to all antifungals. Fluconazole resistance was $5.2 \%$ for C. albicans while it was $2.6 \%$ for Candida tropicalis. Risk factors for invasive candidemia were found to be surgery especially abdominal surgery, central venous catheters, invasive mechanical ventilation, urinary catheterization, hemodialysis, and total parenteral nutrition. ${ }^{164}$

\section{What are the Risk Factors for Invasive Fungal Infections in ICU?}

Risk factors for invasive fungal infections (IFIs) in ICU have been studied extensively. A large retrospective study in 301 surgical ICU patients found the risk factors for IFI to be peripheral and central intravenous catheters, bladder catheters, mechanical ventilation, lack of enteral nutrition and TPN. ${ }^{460}$ In a prospective study of 150 cardiothoracic ICU patients, risk factors for IFIs were prolonged mechanical ventilation ( $\geq 10$ days), hospital-acquired bacterial infection, cardiopulmonary bypass duration greater than $120 \mathrm{~min}$, diabetes mellitus and high APACHE II score (>30). ${ }^{461}$ A systematic review demonstrated that major surgery (OR-7.3), TPN (OR-3.8), fungal colonization with colonization index $>0.5$ (OR-19.1), hemodialysis(OR-3.8), acute renal failure(OR-4.2), severe sepsis, mechanical ventilation $>3$ days, diabetes $(\mathrm{OR}-2.8)$, APACHE 2 score $>16$ (OR-1.03), cardiopulmonary bypass $>120$ min (OR-8.1), use of broad spectrum antibiotics (OR-3), red cell transfusion and central or peripheral venous catheters were significantly associated with IFIs. ${ }^{462}$

\section{Evidence Statement}

Risk factors for invasive fungal infections in nonneutropenic patients in ICU are surgery, total parenteral nutrition, renal replacement therapy, cardiopulmonary bypass > 120 minutes, diabetes mellitus, central venous catheters, urinary catheters, Candida colonisation with colonization index $>0.5$, use of broad-spectrum antibiotics, acute renal failure, mechanical ventilation $>3$ days and APACHE II score $>16$.

\section{What is the Role of Empirical Antifungals in Non-Neutropenic Patients in ICU?}

The advantage of empirical antifungal treatment has already been established in high-risk patients such as cancer patients and solid organ transplant recipients in various studies. ${ }^{463-465}$ However, in non-neutropenic critically ill patients, the definitive evidence for the efficacy of untargeted treatment in terms of prevention of IFIs or mortality benefit has been equivocal. Moreover, studies have shown the potential detrimental effects of the injudicious use of antifungal agents in the form of the emergence of drug resistance, side effects, and financial costs. ${ }^{466-468}$ Several randomized controlled trials have compared empirical antifungals to placebo in nonneutropenic critically ill patients. ${ }^{469-474}$ In an RCT including post-surgery patients, fluconazole reduced the occurrence of candidemia (5.8\% in fluconazole vs. $16 \%$ in placebo) through the mortality rates were similar. ${ }^{469}$ Similarly, use of caspofungin was also associated with a trend towards decreased IFI without any difference in mortality or length of hospital stay. ${ }^{471}$ A systematic review demonstrated that although empirical antifungals in non-neutropenic patients in ICU reduced the incidence of subsequent IFI, it had no impact on mortality. ${ }^{474}$ In a randomized controlled trial involving 260 mechanically ventilated patients with Candida colonization, empirical micafungin administration reduced the rate of subsequent proven IFI (12\% vs. $3 \%$; $p=0.008)$ without any significant mortality benefit. ${ }^{473}$

\section{Evidence Statement}

Empirical antifungals for non-neutropenic patients in ICU routinely has not been associated with a decrease in mortality or hospital length of stay. 
Empirical antifungals in patients at high risk for invasive fungal infections in ICU has been shown to reduce the incidence of subsequent proven invasive fungal infections.

\section{Recommendations}

- We do not recommend the routine use of empirical antifungals in non-neutropenic patients in ICU (1A).

- Empirical antifungals may be considered in critically ill patients with a high risk of invasive fungal infections to reduce the incidence of subsequent invasive fungal infections (1B).

\section{What is the Antifungal Agent of Choice and Duration of Empirical Therapy in Non-neutropenic Patients in ICU?}

The options for antifungal therapy include fluconazole, amphotericin-B, and echinocandins. In a systematic review, empirical use of fluconazole and caspofungin reduced rates of subsequent IFI while micafungin, nystatin, and amphotericin-B did not. ${ }^{474}$ No direct comparative data of efficacy of different antifungals for empirical therapy in non-neutropenic patients in ICU is available. Indian studies have shown an increased prevalence of non-albicans Candida with high rates of fluconazole resistance in the range of 5 to $7 \%{ }^{475}$ Regarding duration of empirical antifungal therapy, and there are no studies directly comparing the different duration of empirical antifungal therapy. Most of the studies have used at least 2 weeks therapy. ${ }^{474}$

\section{Evidence Statement}

Fluconazole and caspofungin are useful as empirical antifungal therapy in non-neutropenic ICU patients at high risk of invasive fungal infection. In India, the rate of fluconazole resistance is up to $7 \%$, especially in nonalbicans candida species.

\section{Recommendations}

- We recommend fluconazole or caspofungin as preferred empirical antifungal agents in nonneutropenic ICU patients at risk for invasive fungal infection (1A).

- Caspofungin may be preferred in areas with high prevalence of fluconazole resistance (1B).

- Micafungin or anidulafungin may be used as alternative agents (3A).

- Recommended duration of empirical antifungal therapy is 2 weeks ( $3 \mathrm{~A})$.

\section{Antibiotic Stewardship}

Antibiotic stewardship program is defined as "coordinated interventions designed to improve and measure the appropriate use of antibiotic agents by promoting the selection of the optimal drug regimen including dosing, duration of therapy, and route of administration." ${ }^{\prime 476}$ An efficient antibiotic stewardship program results in optimum clinical outcomes while reducing adverse effects of unnecessary antibiotic use. Every additional 10 days of antibiotic therapy conferred a 3\% increased risk of an adverse drug event. These adverse effects include the emergence of antibiotic resistance, Clostridium difficile infections and drug toxicity and occurs in $20 \%$ of patients. ${ }^{477} \mathrm{~A}$ structured antibiotic stewardship program requires a multidisciplinary approach. Core elements of antibiotic stewardship program include committed leadership, accountability, expertise in drugs, action, tracking drug resistance patterns, regular reporting and education to clinicians about optimal prescribing. ${ }^{478}$

\section{Does Antibiotic Stewardship Improve Patient Outcome in ICU?}

Antibiotic stewardship programs reduced the duration of antibiotic treatment (1.95 days; 95\% CI 2.22-1.67) and duration of hospital stay (1.12 days, $95 \%$ CI $0.7-1.54$ ) without any significant difference in mortality in a recent systematic review. ${ }^{47}$ In a recent meta-analysis, there was reduced mortality with guideline directed empirical therapy (RR 0.65 , $95 \%$ CI $0.54-0.80, p<0.0001$ ) and antibiotic de-escalation (RR $0.44,0.30-0.66) .{ }^{479}$ Mortality benefit has also been reported in another systematic review (RR $0.68,95 \%$ CI 0.52-0.88). ${ }^{480}$ However, a single non blinded randomized study showed a significantly higher rate of superinfection with the de-escalation of antibiotics as compared to continuation of empirical therapy $(27 \%$ vs. $11 \%$; $p=0.03) .{ }^{481}$

\section{Evidence Statement}

Antibiotic stewardship programs in hospitalized patients are associated with a reduction in a number of antibiotic days, duration of hospital stay and all-cause mortality.

\section{Recommendations}

- All hospitals should have an antibiotic stewardship program including the intensive care units (2A).

\section{What are the Essential Strategies of Antibiotic Stewardship in An ICU Setting?}

Prospective audit-feedback and preauthorization are commonly used strategies of antibiotic stewardship. ${ }^{482-484}$ In prospective audit and feedback, treating clinicians are provided recommendations regarding the appropriateness 
of antibiotics used. Advantages of this strategy include avoidance of delay in antibiotic administration (as the physician is engaged after prescription of antibiotics). Limitations of this strategy include partial compliance (due to voluntary participation of physicians), resource intensive nature, and a longer lag period for visible benefits to become apparent. Prospective audit and feedback strategy resulted in a reduction in the utilization of antibiotics and significant cost reduction. ${ }^{485,486}$ In a systematic review, enabling strategies including feedback resulted in greater efficacy of stewardship interventions. ${ }^{482}$ Preauthorisation, another strategy of antibiotic stewardship, requires approval by the concerned authority before starting antibiotics. ${ }^{483}$ This affects the use of restricted antibiotics only and may result in a potential delay in antibiotic initiation. Without feedback, this may also result in increased use of other antibiotics and hence lead to the selection of different resistance patterns. However, it provides immediate results in terms of reduced antibiotic usage. Other potential drawbacks include the development of negative professional culture because of a breakdown in communication between infectious disease specialists and clinical teams. ${ }^{482}$ Enabling and restrictive strategies have been compared in a quasi-experimental crossover trial using days of antibiotic therapy in both strategies. ${ }^{487}$ In this study involving 2686 patients in pre-prescription authorization (PPA) group and 2693 patients in postprescription review with feedback (PPRF) group, initially antibiotic days of treatment (DOT) remained relatively unchanged in the PPA arm. When changed to the PPRF arm, antibiotic use decreased (-2.45 DOT per 1000 patientdays (PD)] hence concluding that PPRF may have more impact on decreasing days of antibiotic therapy.

\section{Evidence Statement}

Antibiotic stewardship requires a multidisciplinary approach with integration of infectious disease physician, a microbiologist with logistic and financial support from hospital administration. Both enablement and restrictive strategies are useful in improving adherence to antibiotic stewardship programs. Restrictive strategies give immediate results. Enablement practices are more resource intensive. Most studies have used a combination of both the methods and have shown additive effects. Providing feedback to the treating team improves adherence. A single RCT has shown that a restrictive strategy alone may cause a delay in the initiation of antibiotics.

\section{Recommendations}

- Prospective audit of antibiotic use and/or preauthorization (if feasible) along with feedback to the treating team is recommended as part of an antibiotic stewardship program (1A).

\section{What is the Role of Antibiotic Cycling, Intravenous to Oral switch and De-escalation in the ICU?}

Antibiotic cycling refers to withdrawing a specific antibiotic or an antibiotic class from use for a definite period and substituting with another antibiotic or antibiotic class having a similar spectrum of activity. ${ }^{483}$ This is postulated to induce different resistance mechanisms in the microorganisms and hence prevent or reverse the development of antibiotic resistance. There is no compelling evidence on the benefit of antibiotic cycling in terms of clinical endpoints. Several prospective before and after studies without control groups have demonstrated a reduction in the incidence of ventilator-associated pneumonia $(6.7 \%$ with antibiotic cycling as against $11.6 \%$ before the intervention) $)^{489}$ as well as a reduction in colonization. ${ }^{489-491}$ A newer prospective cohort study ${ }^{492}$ comparing antibiotic mixing, and antibiotic cycling found no significant differences in infection rates $(16.6 \%$ and $14.5 \%$, OR 0.9$)$, infection due to target microorganisms $(5.9 \%$ and $5.2 \%$, OR 0.9$)$, hospital length of stay (median 5 days for both groups) or in-hospital mortality (13.9\% and $14.3 \%$, OR 1.03 ).

\section{Evidence Statement}

Antibiotic cycling in the intensive care unit has not been adequately studied in randomized controlled trials. Nonrandomized studies show significant heterogeneity in terms of site of study, a method of cycling and confounders like simultaneous infection control measures being employed. Evidence of benefit of antibiotic cycling is lacking, with few studies demonstrating a reduction in colonization though mortality and length of hospital stay remain unchanged.

\section{Recommendations}

- Antibiotic cycling should not be used as a method of the antibiotic stewardship program (2A).

\section{Scheduled Intravenous to Oral Switch}

Timely switch from intravenous to oral antibiotics has been shown to reduce the cost of health care and length of hospital stay. ${ }^{493-498}$ In case of antibiotics with the availability of equivalent oral formulations, the scheduled switch is easier than in case of broadspectrum antibiotics without oral formulations or precise like piperacillin-tazobactam or meropenem. A multicenter randomized controlled trial done in CAP which evaluated scheduled switch to oral antibiotics after 2 days of intravenous antibiotics found similar 
cure rates, survival or resolution of chest radiology with a significantly lower total cost of care (2953\$ and 5002\$, $\mathrm{p}<0.05) .{ }^{497}$ Oosterheert et al ${ }^{496}$ also found similar results when comparing scheduled switch on day 3 and day 7 with similar cure rates and mortality rates in both groups but with significantly reduced duration of intravenous antibiotics and hospital stay, with differences of 3.4 days and 1.9 days respectively.

\section{Evidence Statement}

Early intravenous to the oral transition of antibiotics reduce hospital length of stay and cost of care. There is no increase in mortality or other adverse events when this is done after assessing as to which patients can be safely transitioned to oral therapy.

\section{Recommendations}

- Antibiotic stewardship programs should implement strategies to improve the timely transition from parenteral to oral antibiotic therapy (2A).

\section{De-escalation in Intensive Care Unit}

Antibiotic de-escalation refers to a strategy of switching from broad-spectrum antimicrobials to a narrower spectrum of antimicrobials. It is recommended to reduce the emergence of multidrug-resistant bacteria as well as costs of health care. In a multicenter randomized controlled trial, de-escalation was associated with a longer ICU stay but similar in-hospital mortality in severe sepsis. ${ }^{481}$ In a recent meta-analysis of 9 studies involving 1873 patients with septic shock, deescalation of antibiotics was associated with a trend towards reduced mortality (RR 0.74, 95\% CI 0.54-1.03). ${ }^{499}$ In another systematic review, de-escalation was associated with lower mortality (RR $0.68 ; 95 \%$ CI 0.52-0.88). ${ }^{480}$

\section{Evidence Statement}

Pooled results from observational studies in an ICU setting do not show any increase in mortality with antibiotic de-escalation while significantly reducing antibiotic exposure days and ICU length of stay.

\section{Recommendations}

- Antibiotic de-escalation in the ICU is recommended as part of an antibiotic stewardship program (2A).

\section{What is the Role of Procalcitonin in Antibiotic De-escalation ICU?}

Procalcitonin is a 116 amino acid precursor to calcitonin. Normal serum or plasma levels of procalcitonin in healthy adults are $<0.05 \mathrm{ng} / \mathrm{mL}$. It can be produced by a variety of cell types in response to inflammatory stimuli, especially of bacterial origin. It does not usually rise significantly in response to viral or noninfectious inflammation and so has the potential to be used as a marker of bacterial infection. The levels in serum are quantified using immunoassay. ${ }^{127}$ Procalcitonin use to guide antibiotic therapy in sepsis in intensive care unit resulted in reduction in antibiotic days (MD-3.19 days, 95\% CI -5.44- - -0.95) duration of hospital stay (MD-3.85 days, 95\% CI $-6.78-0.92)$ as well as a trend towards reduction in duration of ICU stay (MD-2.03 days, 95\% CI -4.19-0.13 days). ${ }^{500}$ Procalcitonin-guided algorithm for antibiotic discontinuation (decrease by $\geq 80 \%$ of peak value, or $\leq 0.5 \mathrm{ng} / \mathrm{mL}$ ) led to reduced antibiotic administration (between-group absolute difference 1.22, 0.65-1.78, p < 0.0001), with significant mortality benefit (20\% vs. $25 \%$; between-group absolute difference 5.4\%, 95\% CI 1.2-9.5, $\mathrm{p}=0.0122) .{ }^{501}$ In a recent Cochrane meta-analysis involving 26 trials, procalcitonin utilization for antibiotic discontinuation was associated with reduced mortality (adjusted OR 0.83, 95\% CI 0.70 to $0.99, \mathrm{p}=$ 0.037). ${ }^{84}$

\section{Evidence Statement}

Implementation of antibiotic de-escalation algorithm based on serial procalcitonin measurements has been shown to reduce mortality, length of ICU stay, the total duration of antibiotic days and health care costs.

\section{Recommendations}

- Procalcitonin based algorithms may be used for antibiotic de-escalation (1A).

\section{Author Details}

${ }^{1,6,10}$ Professor and Head, ${ }^{2}$ Director and HOD, ${ }^{3,5}$ Assistant Professor, ${ }^{4}$ President and Chairman, ${ }^{7}$ Professor, ${ }^{8,9}$ Director

$1,3,5,7,9$ Department of Pulmonary Medicine and Sleep Disorders, All India Institute of Medical Sciences, New Delhi, India, ${ }^{2}$ Department, Neuro-Trauma Unit, Grant Medical Foundation, Ruby Hall Clinic, Pune, Maharashtra, India, ${ }^{4}$ Indian Society of Critical Care Medicine, Medanta Institute of Critical Care and Anesthesiology, Medanta, Haryana, India, ${ }^{6}$ Department of Anaesthesiology, Division of Critical Care Medicine, Critical Care and Pain, Tata Memorial Hospital, Mumbai, Maharashtra, India, ${ }^{8}$ Sanjeevan and MJM Hospital, Pune, Maharashtra, India, ${ }^{10}$ Department of Anaesthesiology, Critical Care and Emergency Services, Bhopal, Madhya Pradesh, India

Other Contributors: Avneet Garg, Dilip Dubey, Hariharan Iyer, Jugendra Singh, Prashuram Bista, Sachin Doddamani, Sandip Agarwal, Shiba Kalyan Biswal, Shweta Bansal, Swapnil Mehta, Sryma PB, Tajammul Hussain Shah, Tejas Suri 


\section{REFERENCES}

1. Álvarez-Lerma F, Grau S. Management of Antimicrobial Use in the Intensive Care Unit. Drugs. 2012;72:447-470.

2. Abdul-Aziz MH, Sulaiman H, Mat-Nor M-B, Rai V, Wong KK, Hasan MS, et al. Beta-Lactam Infusion in Severe Sepsis (BLISS): a prospective, two-centre, open-labelled randomised controlled trial of continuous versus intermittent beta-lactam infusion in critically ill patients with severe sepsis. Intensive Care Med. 2016;42:1535-1545.

3. Dulhunty JM, Roberts JA, Davis JS, Webb SAR, Bellomo $\mathrm{R}$, Gomersall C, et al. A Multicenter Randomized Trial of Continuous versus Intermittent $\beta$-Lactam Infusion in Severe Sepsis. Am J Respir Crit Care Med. 2015;192:1298-1305.

4. Roberts JA, Abdul-Aziz MH, Davis JS, Dulhunty JM, Cotta $\mathrm{MO}$, Myburgh J, et al. Continuous versus Intermittent $\beta$-Lactam Infusion in Severe Sepsis. A Meta-analysis of Individual Patient Data from Randomized Trials. Am J Respir Crit Care Med. 2016;194:681-691.

5. Hao JJ, Chen H, Zhou JX. Continuous versus intermittent infusion of vancomycin in adult patients: A systematic review and meta-analysis. Int J Antimicrob Agents. 2016;47:28-35.

6. Gupta D, Agarwal R, Aggarwal AN, Singh N, Mishra N, Khilnani GC, et al. Guidelines for diagnosis and management of community-and hospital-acquired pneumonia in adults: Joint ICS/NCCP(I) Recommendationss. Lung India. 2012 Jul;29(Suppl 2):S27.

7. Lim WS, Baudouin SV, George RC, Hill AT, Jamieson C, Jeune $\mathrm{IL}$, et al. BTS guidelines for the management of community acquired pneumonia in adults: update 2009. Thorax. 2009;64:iii1-55.

8. Pneumonia: Diagnosis and Management of Community- and Hospital-Acquired Pneumonia in Adults. London: National Institute for Health and Care Excellence (UK); 2014.

9. Woodhead M, Blasi F, Ewig S, Garau J, Huchon G, Ieven M, et al. Guidelines for the management of adult lower respiratory tract infections - Full version. Clin Microbiol Infect. 2011;17:E1-59.

10. Jain S, Self WH, Wunderink RG, Fakhran S, Balk R, Bramley $\mathrm{AM}$, et al. Community-Acquired Pneumonia Requiring Hospitalization among U.S. Adults. N Engl J Med. 2015;373: 415-427.

11. Vardakas KZ, Matthaiou DK, Falagas ME. Incidence, characteristics and outcomes of patients with severe community acquired-MRSA pneumonia. Eur Respir J. 2009;34:1148-1158.

12. Gillet $Y$, Issartel B, Vanhems P, Fournet JC, Lina G, Bes M, et al. Association between Staphylococcus aureus strains carrying gene for Panton-Valentine leukocidin and highly lethal necrotising pneumonia in young immunocompetent patients. Lancet Lond Engl. 2002;359:753-759.

13. Lobo LJ, Reed KD, Wunderink RG. Expanded Clinical Presentation of Community-Acquired Methicillin-Resistant Staphylococcus aureus Pneumonia. CHEST. 2010;138:130-136.

14. Self WH, Wunderink RG, Williams DJ, Zhu Y, Anderson EJ, Balk RA, et al. Staphylococcus aureus Communityacquired Pneumonia: Prevalence, Clinical Characteristics, and Outcomes. Clin Infect Dis Off Publ Infect Dis Soc Am. 2016;63:300-309.

15. Peto L, Nadjm B, Horby P, Ngan TTD, van Doorn R, Van Kinh $\mathrm{N}$, et al. The bacterial aetiology of adult community-acquired pneumonia in Asia: a systematic review. Trans R Soc Trop Med Hyg. 2014;108:326-337.
16. Vanlalruati RSC, Mamta Devi KSH, Singh NB, Singh NT. A study of bacteriological profile (aerobic) and antimicrobial susceptibility of community acquired pneumonia cases in the RIMS hospital. J Commun Dis. 2012;44:47-49.

17. Peto L, Nadjm B, Horby P, Ngan TTD, van Doorn R, Kinh NV, et al. The bacterial aetiology of adult community-acquired pneumonia in Asia: a systematic review. Trans R Soc Trop Med Hyg. 2014;108:326-337.

18. Chawla R, Kansal S, Jain A, Jibhkate B, Chauhan M. Predictors of mortality and length of stay in hospitalized cases of 2009 influenza A (H1N1): Experiences of a tertiary care center. Indian J Crit Care Med. 2013;17:275.

19. Krishnappa L, Marie M, John J, Dabwan K, Shashidhar P. Serological and molecular capsular typing, antibiotic susceptibility of Streptococcus pneumoniae isolates from invasive and non-invasive infections. Acta Microbiol Immunol Hung. 2014;61:173-179.

20. Suryam V, Bhatti VK, Kulkarni A, Mahen A, Nair V. Outbreak control of community acquired pneumonia in a large military training institution. Med J Armed Forces India. 2015;71:33-37.

21. Thomas K. Prospective multicentre hospital surveillance of Streptococcus pneumoniae disease in India. The Lancet. 1999;353:1216-1221.

22. Thomas K, Mukkai Kesavan L, Veeraraghavan B, Jasmine S, Jude J, Shubankar M, et al. Invasive pneumococcal disease associated with high case fatality in India. J Clin Epidemiol. 2013;66:36-43.

23. Chudasama R, Patel U, Verma P, Amin C, Savaria D, Ninama $\mathrm{R}$, et al. Clinico-epidemiological features of the hospitalized patients with 2009 pandemic influenza A (H1N1) virus infection in Saurashtra region, India (September, 2009 to February, 2010). Lung India: official organ of Indian Chest Society. 2011 Jan;28(1):11.

24. Menon R, Menon U, George A. Etiology and anti-microbial sensitivity of organisms causing community acquired pneumonia: A single hospital study. J Fam Med Prim Care. 2013;2:244.

25. Song JH, Oh WS, Kang CI, Chung DR, Peck KR, Ko KS, et al. Epidemiology and clinical outcomes of community-acquired pneumonia in adult patients in Asian countries: a prospective study by the Asian network for surveillance of resistant pathogens. Int J Antimicrob Agents. 2008;31:107-114.

26. Hageman JC, Uyeki TM, Francis JS, Jernigan DB, Wheeler JG, Bridges $C B$, et al. Severe Community-acquired Pneumonia Due to Staphylococcus aureus, 2003-04 Influenza Season. Emerg Infect Dis. 2006;12:894-899.

27. Tong SY, Kearns AM. Community-associated MRSA from the Indian subcontinent. Lancet Infect Dis. 2013;13:734-735.

28. Khadanga $S$, Thatoi $P$, Behera $S$, Karuna T. Changing bacteriological profile and mortality trends in community acquired pneumonia. J Glob Infect Dis. 2014;6:186.

29. Bansal S, Kashyap S, Pal LS, Goel A. Clinical and Bacteriological Profile of Community Acquired Pneumonia in Shimla, Himachal Pradesh. Indian Journal of Chest Diseases and Allied Sciences. 2004 Mar;46(1):17-22.

30. Song JH, Oh WS, Kang CI, Chung DR, Peck KR, Ko KS, et al. Epidemiology and clinical outcomes of community-acquired pneumonia in adult patients in Asian countries: a prospective study by the Asian network for surveillance of resistant pathogens. Int J Antimicrob Agents. 2008;31:107-114.

31. Naik M, Dhobi G, Shah B, Singh G. Bacteriological and clinical profile of Community acquired pneumonia in hospitalized patients. Lung India. 2010;27:54. 
32. Madan R, Kairo AK, Sharma A, Roy S, Singh S, Singh L, et al. Aspiration pneumonia related deaths in head and neck cancer patients: a retrospective analysis of risk factors from a tertiary care centre in North India. J Laryngol Otol. 2015;129:710-714.

33. Lakshmaiah KC, Sirsath NT, Subramanyam JR, Govind BK, Lokanatha D, Shenoy AM. Aspiration in Head and Neck Cancer Patients: A Single Centre Experience of Clinical Profile, Bacterial Isolates and Antibiotic Sensitivity Pattern. Indian J Otolaryngol Head Neck Surg. 2013;65:144-149.

34. Mathai AS, Oberoi A, Madhavan S, Kaur P. Acinetobacter infections in a tertiary level intensive care unit in northern India: Epidemiology, clinical profiles and outcomes. J Infect Public Health. 2012;5:145-152.

35. Prasad P, Bhat S. Clinicomicrobiological study of communityacquired pneumonia. Lung India. 2017;34:491.

36. Deva A, Prasad SR, Madappa BP, Junjegowda K, Bachu RPN. Pneumococcal infections at a rural tertiary care hospital: a seven year study on isolation rate, clinical spectrum and antibiogram. J Clin Diagn Res JCDR. 2014;8:50-52.

37. Molander V, Elisson C, Balaji V, Backhaus E, John J, Vargheese $\mathrm{R}$, et al. Invasive pneumococcal infections in Vellore, India: clinical characteristics and distribution of serotypes. BMC Infect Dis. 2013;13:532.

38. Bharadwaj R, Bal AM, Joshi SA, Kagal A, Pol SS, Garad G, et al. An urban outbreak of leptospirosis in Mumbai, India. Jpn J Infect Dis. 2002; 55(6):194-196.

39. Yende S, van der Poll T, Lee M, Huang DT, Newman AB, Kong $\mathrm{L}$, et al. The influence of pre-existing diabetes mellitus on the host immune response and outcome of pneumonia: analysis of two multicentre cohort studies. Thorax. 2010;65:870-877.

40. Para RA, Fomda BA, Jan RA, Shah S, Koul PA. Microbial etiology in hospitalized North Indian adults with communityacquired pneumonia. Lung India Off Organ Indian Chest Soc. 2018;35:108-1015.

41. Prina E, Ranzani OT, Polverino E, Cillóniz C, Ferrer M, Fernandez L, et al. Risk factors associated with potentially antibiotic-resistant pathogens in community-acquired pneumonia. Ann Am Thorac Soc. 2015;12:153-160.

42. Sibila O, Laserna E, Maselli DJ, Fernandez JF, Mortensen EM, Anzueto A, et al. Risk factors and antibiotic therapy in P.aeruginosa community-acquired pneumonia. Respirol Carlton Vic. 2015;20:660-666.

43. Falguera M, Carratalà J, Ruiz-Gonzalez A, Garcia-Vidal C, Gazquez I, Dorca J, et al. Risk factors and outcome of community-acquired pneumonia due to Gram-negative bacilli. Respirol Carlton Vic. 2009;14:105-111.

44. Cilloniz C, Martin-Loeches I, Garcia-Vidal C, San Jose A, Torres A. Microbial Etiology of Pneumonia: Epidemiology, Diagnosis and Resistance Patterns. Int J Mol Sci. 2016;17(12):2120.

45. Yu KT, Wyer PC. Evidence Behind the 4-Hour Rule for Initiation of Antibiotic Therapy in Community-Acquired Pneumonia. Ann Emerg Med. 2008;51:651-662.e2.

46. Sterling SA, Miller WR, Pryor J, Puskarich MA, Jones AE. The Impact of Timing of Antibiotics on Outcomes in Severe Sepsis and Septic Shock: A Systematic Review and Meta-Analysis. Crit Care Med. 2015;43:1907-1915.

47. Liu VX, Fielding-Singh V, Greene JD, Baker JM, Iwashyna TJ, Bhattacharya J, et al. The Timing of Early Antibiotics and Hospital Mortality in Sepsis. Am J Respir Crit Care Med. 2017;196:856-863.

48. Falguera M, Ruiz-González A, Schoenenberger JA, Touzón C, Gázquez I, Galindo C, et al. Prospective, randomised study to compare empirical treatment versus targeted treatment on the basis of the urine antigen results in hospitalised patients with community-acquired pneumonia. Thorax. 2010;65:101-106.

49. Eerden MM van der, Vlaspolder F, Graaff CS de, Groot $\mathrm{T}$, Bronsveld W, Jansen HM, et al. Comparison between pathogen directed antibiotic treatment and empirical broad spectrum antibiotic treatment in patients with community acquired pneumonia: a prospective randomised study. Thorax. 2005;60:672-678.

50. Uematsu $H$, Hashimoto $H$, Iwamoto $T$, Horiguchi $H$, Yasunaga $\mathrm{H}$. Impact of guideline-concordant microbiological testing on outcomes of pneumonia. Int J Qual Health Care. 2014;26:100-107.

51. Lidman C, Burman LG, Lagergren Å, Örtqvist Å. Limited Value of Routine Microbiological Diagnostics in Patients Hospitalized for Community-acquired Pneumonia. Scand J Infect Dis. 2002;34:873-879.

52. Meehan TP, Fine MJ, Krumholz HM, Scinto JD, Galusha DH, Mockalis JT, et al. Quality of care, process, and outcomes in elderly patients with pneumonia. JAMA. 1997;278: 2080-2084.

53. Sligl WI, Asadi L, Eurich DT, Tjosvold L, Marrie TJ, Majumdar SR. Macrolides and mortality in critically ill patients with community-acquired pneumonia: a systematic review and meta-analysis. Crit Care Med. 2014;42:420-432.

54. Adrie C, Schwebel C, Garrouste-Orgeas M, Vignoud L, Planquette B, Azoulay E, et al. Initial use of one or two antibiotics for critically ill patients with community-acquired pneumonia: impact on survival and bacterial resistance. Crit Care. 2013;17:R265.

55. Gattarello S, Borgatta B, Solé-Violán J, Vallés J, Vidaur L, Zaragoza R, et al. Decrease in Mortality in Severe CommunityAcquired Pneumococcal Pneumonia. Chest. 2014;146:22-31.

56. Lee JH, Kim HJ, Kim YH. Is $\beta$-Lactam Plus Macrolide More Effective than $\beta$-Lactam Plus Fluoroquinolone among Patients with Severe Community-Acquired Pneumonia?: a Systemic Review and Meta-Analysis. J Korean Med Sci. 2017;32: 77-84.

57. Chang KC, Leung CC, Yew WW, Lau TY, Leung WM, Tam CM, et al. Newer fluoroquinolones for treating respiratory infection: do they mask tuberculosis? Eur Respir J. 2010;35:606-613.

58. von Baum H, Welte T, Marre R, Suttorp N, Ewig S, CAPNETZ study group. Community-acquired pneumonia through Enterobacteriaceae and Pseudomonas aeruginosa: Diagnosis, incidence and predictors. Eur Respir J. 2010;35:598-605.

59. Arancibia F, Bauer TT, Ewig S, Mensa J, Gonzalez J, Niederman MS, et al. Community-acquired pneumonia due to gramnegative bacteria and Pseudomonas aeruginosa: incidence, risk, and prognosis. Arch Intern Med. 2002;162:1849-1858.

60. Cillóniz C, Gabarrús A, Ferrer M, Puig de la Bellacasa J, Rinaudo M, Mensa J, et al. Community-Acquired Pneumonia Due to Multidrug- and Non-Multidrug-Resistant Pseudomonas aeruginosa. Chest. 2016;150:415-425.

61. Rello J, Rodriguez A, Torres A, Roig J, Sole-Violan J, GarnachoMontero J, et al. Implications of COPD in patients admitted to the intensive care unit by community-acquired pneumonia. Eur Respir J. 2006;27:1210-1216.

62. Vila-Corcoles A, Ochoa-Gondar O, Rodriguez-Blanco T, Raga-Luria X, Gomez-Bertomeu F, EPIVAC Study Group. Epidemiology of community-acquired pneumonia in older adults: a population-based study. Respir Med. 2009;103:309-316.

63. Vardakas KZ, Matthaiou DK, Falagas ME. Incidence, characteristics and outcomes of patients with severe 
community acquired-MRSA pneumonia. Eur Respir J. 2009;34:1148-1158.

64. Carballo N, De Antonio-Cuscó M, Echeverría-Esnal D, Luque S, Salas E, Grau S. Community-acquired pneumonia caused by methicillin-resistant Staphylococcus aureus in critically-ill patients: systematic review. Farm Hosp Organo Of Expresion Cient Soc Espanola Farm Hosp. 2017;41:187-203.

65. Mandell LA, Wunderink RG, Anzueto A, Bartlett JG, Campbell GD, Dean NC, et al. Infectious Diseases Society of America/ American Thoracic Society consensus guidelines on the management of community-acquired pneumonia in adults. Clin Infect Dis Off Publ Infect Dis Soc Am. 2007;44 Suppl 2:S27-S72.

66. Lorber B, Swenson RM. Bacteriology of aspiration pneumonia. A prospective study of community- and hospital-acquired cases. Ann Intern Med. 1974;81:329-331.

67. Cesar L, Gonzalez C, Calia FM. Bacteriologic flora of aspiration-induced pulmonary infections. Arch Intern Med. 1975;135:711-714.

68. Brook I, Frazier EH. Aerobic and anaerobic microbiology of empyema. A retrospective review in two military hospitals. Chest. 1993;103:1502-1507.

69. Bartlett JG. Anaerobic Bacterial Infections of the Lung and Pleural Space. Clin Infect Dis. 1993;16:S248-S255.

70. Tahon-Castel MM, Beerens H. Anaerobiosis of bucco-dental origin. Rev Stomatoodontol Nord Fr. 1965;20:13-22.

71. Gudiol F, Manresa F, Pallares R, Dorca J, Rufi G, Boada J, et al. Clindamycin vs penicillin for anaerobic lung infections. High rate of penicillin failures associated with penicillin-resistant Bacteroides melaninogenicus. Arch Intern Med. 1990;150: 2525-2529.

72. Bartlett JG. The Role of Anaerobic Bacteria in Lung Abscess. Clin Infect Dis. 2005;40:923-925.

73. Mori T, Ebe T, Takahashi M, Isonuma H, Ikemoto H, Oguri T. Lung Abscess: Analysis of 66 Cases from 1979 to 1991. Intern Med. 1993;32:278-284.

74. Wang JL, Chen KY, Fang CT, Hsueh PR, Yang PC, Chang SC. Changing bacteriology of adult community-acquired lung abscess in Taiwan: Klebsiella pneumoniae versus anaerobes. Clin Infect Dis Off Publ Infect Dis Soc Am. 2005;40:915-922.

75. Yamasaki K, Kawanami T, Yatera K, Fukuda K, Noguchi S, Nagata $S$, et al. Significance of Anaerobes and Oral Bacteria in Community-Acquired Pneumonia. PLoS ONE. 2013;8: e63103.

76. Griffith DE, Aksamit T, Brown-Elliott BA, Catanzaro A, Daley C, Gordin F, et al. An official ATS/IDSA statement: diagnosis, treatment, and prevention of nontuberculous mycobacterial diseases. Am J Respir Crit Care Med. 2007;175:367-416.

77. Levison ME, Mangura CT, Lorber B, Abrutyn E, Pesanti EL, Levy RS, et al. Clindamycin compared with penicillin for the treatment of anaerobic lung abscess. Ann Intern Med. 1983;98:466-471.

78. Kadowaki M, Demura Y, Mizuno S, Uesaka D, Ameshima S, Miyamori I, et al. Reappraisal of clindamycin IV monotherapy for treatment of mild-to-moderate aspiration pneumonia in elderly patients. Chest. 2005;127:1276-1282.

79. Allewelt M, Schüler P, Bölcskei PL, Mauch H, Lode H, Study Group on Aspiration Pneumonia. Ampicillin + sulbactam vs clindamycin +/- cephalosporin for the treatment of aspiration pneumonia and primary lung abscess. Clin Microbiol Infect Off Publ Eur Soc Clin Microbiol Infect Dis. 2004;10: 163-170.
80. Ott SR, Allewelt M, Lorenz J, Reimnitz P, Lode H, German Lung Abscess Study Group. Moxifloxacin vs ampicillin/ sulbactam in aspiration pneumonia and primary lung abscess. Infection. 2008;36:23-30.

81. Sun T, Sun L, Wang R, Ren X, Sui DJ, Pu C, et al. Clinical efficacy and safety of moxifloxacin versus levofloxacin plus metronidazole for community-acquired pneumonia with aspiration factors. Chin Med J (Engl). 2014;127:1201-1205.

82. Dunbar LM, Wunderink RG, Habib MP, Smith LG, Tennenberg AM, Khashab MM, et al. High-dose, short-course levofloxacin for community-acquired pneumonia: a new treatment paradigm. Clin Infect Dis Off Publ Infect Dis Soc Am. 2003;37:752-760.

83. Choudhury G, Mandal P, Singanayagam A, Akram AR, Chalmers JD, Hill AT. Seven-day antibiotic courses have similar efficacy to prolonged courses in severe communityacquired pneumonia--a propensity-adjusted analysis. Clin Microbiol Infect Off Publ Eur Soc Clin Microbiol Infect Dis. 2011;17:1852-1858.

84. Schuetz P, Wirz Y, Sager R, Christ-Crain M, Stolz D, Tamm $\mathrm{M}$, et al. Procalcitonin to initiate or discontinue antibiotics in acute respiratory tract infections. Cochrane Database Syst Rev. 2017;10:CD007498.

85. Kalil AC, Metersky ML, Klompas M, Muscedere J, Sweeney DA, Palmer LB, et al. Management of Adults With Hospitalacquired and Ventilator-associated Pneumonia: 2016 Clinical Practice Guidelines by the Infectious Diseases Society of America and the American Thoracic Society. Clin Infect Dis Off Publ Infect Dis Soc Am. 2016;63:e61-111.

86. Hayashi $Y$, Morisawa K, Klompas M, Jones M, Bandeshe $H$, Boots R, et al. Toward improved surveillance: the impact of ventilator-associated complications on length of stay and antibiotic use in patients in intensive care units. Clin Infect Dis Off Publ Infect Dis Soc Am. 2013;56:471-477.

87. Craven DE, De Rosa FG, Thornton D. Nosocomial pneumonia: emerging concepts in diagnosis, management, and prophylaxis. Curr Opin Crit Care. 2002;8:421-429.

88. Torres A, Serra-Batlles J, Ros E, Piera C, Puig de la Bellacasa $\mathrm{J}$, Cobos A, et al. Pulmonary aspiration of gastric contents in patients receiving mechanical ventilation: the effect of body position. Ann Intern Med. 1992;116:540-543.

89. Safdar N, Dezfulian C, Collard HR, Saint S. Clinical and economic consequences of ventilator-associated pneumonia: a systematic review. Crit Care Med. 2005;33:2184-2193.

90. Kollef MH, Shorr A, Tabak YP, Gupta V, Liu LZ, Johannes RS. Epidemiology and outcomes of health-care-associated pneumonia: results from a large US database of culturepositive pneumonia. Chest. 2005;128:3854-3862.

91. Sievert DM, Ricks P, Edwards JR, Schneider A, Patel J, Srinivasan A, et al. Antimicrobial-resistant pathogens associated with healthcare-associated infections: summary of data reported to the National Healthcare Safety Network at the Centers for Disease Control and Prevention, 2009-2010. Infect Control Hosp Epidemiol. 2013;34:1-14.

92. Chung DR, Song JH, Kim SH, Thamlikitkul V, Huang SG, Wang $\mathrm{H}$, et al. High prevalence of multidrug-resistant nonfermenters in hospital-acquired pneumonia in Asia. Am J Respir Crit Care Med. 2011;184:1409-1417.

93. Inchai J, Liwsrisakun C, Theerakittikul T, Chaiwarith R, Khositsakulchai W, Pothirat C. Risk factors of multidrugresistant, extensively drug-resistant and pandrug-resistant Acinetobacter baumannii ventilator-associated pneumonia 
in a Medical Intensive Care Unit of University Hospital in Thailand. J Infect Chemother. 2015;21:570-574.

94. Ranjan N, Chaudhary U, Chaudhry D, Ranjan K. Ventilatorassociated pneumonia in a tertiary care intensive care unit: Analysis of incidence, risk factors and mortality. Indian J Crit Care Med. 2014;18:200.

95. Mathur P, Tak V, Gunjiyal J, Nair SA, Lalwani S, Kumar S, et al. Device-associated infections at a level-1 trauma centre of a developing Nation: Impact of automated surveillance, training and feedbacks. Indian J Med Microbiol. 2015; 33:51.

96. Ahmed NH, Hussain T, Biswal I. Antimicrobial resistance of bacterial isolates from respiratory secretions of ventilated patients in a multi-specialty hospital. Avicenna J Med. 2015;5:74-78.

97. Joseph NM, Sistla S, Dutta TK, Badhe AS, Parija SC. Ventilatorassociated pneumonia in a tertiary care hospital in India: incidence and risk factors. J Infect Dev Ctries. 2009;3:771-777.

98. Goel V, Hogade SA, Karadesai SG. Ventilator associated pneumonia in a medical intensive care unit: Microbial aetiology, susceptibility patterns of isolated microorganisms and outcome. Indian J Anaesth. 2012;56:558.

99. Gurjar M, Saigal S, Baronia AK, Rao BP, Azim A, Poddar B, et al. Carbapenem-resistant Acinetobacter ventilator-associated pneumonia: Clinical characteristics and outcome. Indian J Crit Care Med Peer-Rev Off Publ Indian Soc Crit Care Med. 2013;17:129-134.

100. Langer M, Cigada M, Mandelli M, Mosconi P, Tognoni G. Early onset pneumonia: a multicenter study in intensive care units. Intensive Care Med. 1987;13:342-346.

101. Giantsou E, Liratzopoulos N, Efraimidou E, Panopoulou M, Alepopoulou E, Kartali-Ktenidou S, et al. Both early-onset and late-onset ventilator-associated pneumonia are caused mainly by potentially multiresistant bacteria. Intensive Care Med. 2005;31:1488-1494.

102. Gastmeier P, Sohr D, Geffers C, Rüden H, Vonberg RP, Welte T. Early- and Late-Onset Pneumonia: Is This Still a Useful Classification? Antimicrob Agents Chemother. 2009;53:2714-2718.

103. Trouillet JL, Chastre J, Vuagnat A, Joly-Guillou ML, Combaux D, Dombret MC, et al. Ventilator-associated pneumonia caused by potentially drug-resistant bacteria. Am J Respir Crit Care Med. 1998;157:531-539.

104. Goel V, Hogade SA, Karadesai SG. Ventilator associated pneumonia in a medical intensive care unit: Microbial aetiology, susceptibility patterns of isolated microorganisms and outcome. Indian J Anaesth. 2012;56:558.

105. Bouza E, Giannella M, Bunsow E, Torres MV, Pérez Granda MJ, Martín-Rabadán P, et al. Ventilator-associated pneumonia due to meticillin-resistant Staphylococcus aureus: risk factors and outcome in a large general hospital. J Hosp Infect. 2012;80: 150-155.

106. Wooten DA, Winston LG. Risk factors for methicillin-resistant Staphylococcus aureus in patients with community-onset and hospital-onset pneumonia. Respir Med. 2013;107:1266-1270.

107. Moreira MR, Filho PPG. Multidrug-resistant pathogens causing ventilator-associated pneumonia: Risk factors, empirical antimicrobial therapy and outcome of patients in an intensive care unit (ICU) of a Brazilian university hospital. Int J Med Med Sci. 2012;4:204-210.

108. Kuti EL, Patel AA, Coleman CI. Impact of inappropriate antibiotic therapy on mortality in patients with ventilator-associated pneumonia and blood stream infection: a meta-analysis. J Crit Care. 2008;23:91-100.

109. Arthur LE, Kizor RS, Selim AG, van Driel ML, Seoane L. Antibiotics for ventilator-associated pneumonia. Cochrane Database Syst Rev. 2016;10:CD004267.

110. Walkey AJ, O'Donnell MR, Wiener RS. Linezolid vs glycopeptide antibiotics for the treatment of suspected methicillin-resistant Staphylococcus aureus nosocomial pneumonia: a meta-analysis of randomized controlled trials. Chest. 2011;139:1148-1155.

111. Kalil AC, Klompas M, Haynatzki G, Rupp ME. Treatment of hospital-acquired pneumonia with linezolid or vancomycin: a systematic review and meta-analysis. BMJ Open. 2013;3:e003912.

112. Kollef MH, Chastre J, Fagon JY, François B, Niederman MS, Rello J, et al. Global prospective epidemiologic and surveillance study of ventilator-associated pneumonia due to Pseudomonas aeruginosa. Crit Care Med. 2014;42:2178-2187.

113. Parker CM, Kutsogiannis J, Muscedere J, Cook D, Dodek P, Day AG, et al. Ventilator-associated pneumonia caused by multidrugresistant organisms or Pseudomonas aeruginosa: prevalence, incidence, risk factors, and outcomes. J Crit Care. 2008;23:18-26.

114. Rello J, Ausina V, Ricart M, Puzo C, Quintana E, Net A, et al. Risk factors for infection by Pseudomonas aeruginosa in patients with ventilator-associated pneumonia. Intensive Care Med. 1994;20:193-198.

115. Montero M, Sala M, Riu M, Belvis F, Salvado M, Grau S, et al. Risk factors for multidrug-resistant Pseudomonas aeruginosa acquisition. Impact of antibiotic use in a double case-control study. Eur J Clin Microbiol Infect Dis Off Publ Eur Soc Clin Microbiol. 2010;29:335-339.

116. Capellier G, Mockly H, Charpentier C, Annane D, Blasco G, Desmettre T, et al. Early-onset ventilator-associated pneumonia in adults randomized clinical trial: comparison of 8 versus 15 days of antibiotic treatment. PloS One. 2012;7:e41290.

117. Micek ST, Ward S, Fraser VJ, Kollef MH. A randomized controlled trial of an antibiotic discontinuation policy for clinically suspected ventilator-associated pneumonia. Chest. 2004;125:1791-1799.

118. Dimopoulos G, Poulakou G, Pneumatikos IA, Armaganidis A, Kollef MH, Matthaiou DK. Short- vs long-duration antibiotic regimens for ventilator-associated pneumonia: a systematic review and meta-analysis. Chest. 2013;144:1759-1767.

119. Doré P, Robert R, Grollier G, Rouffineau J, Lanquetot H, Charrière JM, et al. Incidence of anaerobes in ventilatorassociated pneumonia with use of a protected specimen brush. Am J Respir Crit Care Med. 1996;153:1292-1298.

120. Marik PE, Careau P. The role of anaerobes in patients with ventilator-associated pneumonia and aspiration pneumonia: a prospective study. CHEST J. 1999;115:178-183.

121. Robertu R, Grollier G, Doré $\mathrm{P}$, Hira M, Ferrand E, Fauchère JL. Nosocomial pneumonia with isolation of anaerobic bacteria in ICU patients: therapeutic considerations and outcome. J Crit Care. 1999;14:114-119.

122. Mokhless NA-S, El-Mofty MF, Hanafi NF, Muhammad A, Asser SL. Atypical Bacteria in Ventilator Associated Pneumonia; an Egyptian University Hospital Experience. J Am Sci. 2010;6:1074-1079.

123. el-Ebiary M, Torres A, González J, de la Bellacasa JP, García C, Jiménez de Anta MT, et al. Quantitative cultures of endotracheal 
aspirates for the diagnosis of ventilator-associated pneumonia. Am Rev Respir Dis. 1993;148:1552-1557.

124. Apfalter P, Stoiser B, Barousch W, Nehr M, Kramer L, Burgmann $\mathrm{H}$. Community-acquired bacteria frequently detected by means of quantitative polymerase chain reaction in nosocomial early-onset ventilator-associated pneumonia. Crit Care Med. 2005;33:1492-1498.

125. Carratala J, Gudiol F, Pallares R, Dorca J, Verdaguer R, Ariza $\mathrm{J}$, et al. Risk factors for nosocomial Legionella pneumophila pneumonia. Am J Respir Crit Care Med. 1994;149:625-629.

126. American Thoracic Society, Infectious Diseases Society of America. Guidelines for the management of adults with hospitalacquired, ventilator-associated, and healthcare-associated pneumonia. Am J Respir Crit Care Med. 2005;171:388-416.

127. Schneider HG, Lam QT. Procalcitonin for the clinical laboratory: a review. Pathology (Phila). 2007;39:383-390.

128. Stolz D, Smyrnios N, Eggimann P, Pargger H, Thakkar N, Siegemund $\mathrm{M}$, et al. Procalcitonin for reduced antibiotic exposure in ventilator-associated pneumonia: a randomised study. Eur Respir J. 2009;34:1364-1375.

129. Bouadma L, Luyt CE, Tubach F, Cracco C, Alvarez A, Schwebel $\mathrm{C}$, et al. Use of procalcitonin to reduce patients' exposure to antibiotics in intensive care units (PRORATA trial): a multicentre randomised controlled trial. Lancet Lond Engl. 2010;375:463-474.

130. Pugh R, Grant C, Cooke RPD, Dempsey G. Short-course versus prolonged-course antibiotic therapy for hospital-acquired pneumonia in critically ill adults. Cochrane Database Syst Rev. 2015;CD007577.

131. Schuetz P, Briel M, Christ-Crain M, Stolz D, Bouadma L, Wolff $\mathrm{M}$, et al. Procalcitonin to guide initiation and duration of antibiotic treatment in acute respiratory infections: an individual patient data meta-analysis. Clin Infect Dis Off Publ Infect Dis Soc Am. 2012;55:651-662.

132. Torres A, Niederman MS, Chastre J, Ewig S, FernandezVandellos P, Hanberger $\mathrm{H}$, et al. International ERS/ESICM/ ESCMID/ALAT guidelines for the management of hospitalacquired pneumonia and ventilator-associated pneumonia: Guidelines for the management of hospital-acquired pneumonia (HAP)/ventilator-associated pneumonia (VAP) of the European Respiratory Society (ERS), European Society of Intensive Care Medicine (ESICM), European Society of Clinical Microbiology and Infectious Diseases (ESCMID) and Asociación Latinoamericana del Tórax (ALAT). Eur Respir J. 2017;50:1700582.

133. A Iannella $\mathrm{H}, \mathrm{M}$ Luna $\mathrm{C}$. Treatment failure in ventilator associated pneumonia. Curr Respir Med Rev. 2012;8:239-244.

134. Luna CM, Blanzaco D, Niederman MS, Matarucco W, Baredes NC, Desmery P, et al. Resolution of ventilator-associated pneumonia: prospective evaluation of the clinical pulmonary infection score as an early clinical predictor of outcome. Crit Care Med. 2003;31:676-682.

135. Dennesen PJ, van der Ven AJ, Kessels AG, Ramsay G, Bonten MJ. Resolution of infectious parameters after antimicrobial therapy in patients with ventilator-associated pneumonia. Am J Respir Crit Care Med. 2001;163:1371-1375.

136. Montravers P, Fagon JY, Chastre J, Lecso M, Dombret MC, Trouillet JL, et al. Follow-up protected specimen brushes to assess treatment in nosocomial pneumonia. Am Rev Respir Dis. 1993;147:38-44.

137. Ioanas M, Ferrer M, Cavalcanti M, Ferrer R, Ewig S, Filella $\mathrm{X}$, et al. Causes and predictors of nonresponse to treatment of intensive care unit-acquired pneumonia. Crit Care Med. 2004;32:938-945.

138. Luna CM, Aruj P, Niederman MS, Garzón J, Violi D, Prignoni A, et al. A ppropriateness and delay to initiate therapy in ventilatorassociated pneumonia. Eur Respir J. 2006;27:158-164.

139. Mittal G, Gaind R, Verma PK, Deb M. Central venous catheterrelated bloodstream infections in an intensive care unit from a tertiary care teaching hospital in India. International Journal of Infection Control. 2016 Mar 28;12(1).

140. Gahlot R, Nigam C, Kumar V, Yadav G, Anupurba S. Catheterrelated bloodstream infections. Int J Crit Illn Inj Sci. 2014;4:161.

141. Mermel LA, Allon M, Bouza E, Craven DE, Flynn P, O'Grady NP, et al. Clinical Practice Guidelines for the Diagnosis and Management of Intravascular Catheter-Related Infection: 2009 Update by the Infectious Diseases Society of America. Clin Infect Dis. 2009;49:1-45.

142. National Nosocomial Infections Surveillance (NNIS) System Report, Data Summary from January 1990-May 1999, Issued June 1999. Am J Infect Control. 1999;27:520-532.

143. National Nosocomial Infections Surveillance System. National Nosocomial Infections Surveillance (NNIS) System Report, data summary from January 1992 through June 2004, issued October 2004. Am J Infect Control. 2004;32:470-485.

144. Chitnis AS, Edwards JR, Ricks PM, Sievert DM, Fridkin SK, Gould CV. Device-Associated Infection Rates, Device Utilization, and Antimicrobial Resistance in Long-Term Acute Care Hospitals Reporting to the National Healthcare Safely Network, 2010. Infect Control Hosp Epidemiol. 2012;33:993-1000.

145. Vincent JL. International Study of the Prevalence and Outcomes of Infection in Intensive Care Units. JAMA. 2009;302:2323.

146. Lorente L, Henry C, Martín MM, Jiménez A, Mora ML. Central venous catheter-related infection in a prospective and observational study of 2,595 catheters. Crit Care. 2005;9:R631-635.

147. Hajjej Z, Nasri M, Sellami W, Gharsallah H, Labben I, Ferjani $\mathrm{M}$. Incidence, risk factors and microbiology of central vascular catheter-related bloodstream infection in an intensive care unit. J Infect Chemother. 2014;20:163-168.

148. Rosenthal VD. Device-Associated Nosocomial Infections in 55 Intensive Care Units of 8 Developing Countries. Ann Intern Med. 2006;145:582.

149. Tarpatzi A, Avlamis A, Papaparaskevas J, Daikos GL, Stefanou I, Katsandri A, et al. Incidence and risk factors for central vascular catheter-related bloodstream infections in a tertiary care hospital. New Microbiol. 2012;35:429-437.

150. Chen HS, Wang FD, Lin M, Lin YC, Huang LJ, Liu CY. Risk factors for central venous catheter-related infections in general surgery. J Microbiol Immunol Infect Wei Mian Yu Gan Ran Za Zhi. 2006;39:231-236.

151. Agarwal R, Gupta D, Ray P, Aggarwal A, Jindal S. Epidemiology, risk factors and outcome of nosocomial infections in a Respiratory Intensive Care Unit in North India. J Infect. 2006;53:98-105.

152. Haq J, Fatema K, Faruq M, Mansur F, Barai L, Karim M. Intravascular catheter related infections and antimicrobial susceptibility pattern of isolated bacteria in a tertiary care hospital of Bangladesh. Indian J Med Microbiol. 2014; 32:68.

153. Chin BS, Han SH, Lee HS, Jeong SJ, Choi H, Kim CO, et al. Risk factors for recurrent catheter-related infections after catheterrelated bloodstream infections. Int J Infect Dis. 2010;14: e16-21. 
154. Peng S, Lu Y. Clinical epidemiology of central venous catheterrelated bloodstream infections in an intensive care unit in China. J Crit Care. 2013;28:277-283.

155. Bicudo D, Batista R, Furtado GH, Sola A, Medeiros EAS de. Risk factors for catheter-related bloodstream infection: a prospective multicenter study in Brazilian intensive care units. Braz J Infect Dis. 2011;15:328-331.

156. Oncü S, Ozsüt H, Yildirim A, Ay P, Cakar N, Eraksoy H, et al. Central venous catheter related infections: risk factors and the effect of glycopeptide antibiotics. Ann Clin Microbiol Antimicrob. 2003;2:1-6.

157. Parameswaran R, Sherchan JB, Varma D M, Mukhopadhyay C, Vidyasagar S. Intravascular catheter-related infections in an Indian tertiary care hospital. J Infect Dev Ctries. 2011;5:452-458.

158. Çaylan HR, Yilmaz G, Sözen EE, Aydin K, Köksal İ. Incidence and risk factors for bloodstream infections stemming from temporary hemodialysis catheters. Turk J Med Sci. 2010;40:835-841.

159. A report from the NNIS System. National Nosocomial Infections Surveillance (NNIS) System Report, data summary from January 1992 through June 2004, issued October 2004. Am J Infect Control. 2004;32:470-485.

160. Kaur M, Gupta V, Gombar S, Chander J, Sahoo T. Incidence, risk factors, microbiology of venous catheter associated bloodstream infections - A prospective study from a tertiary care hospital. Indian J Med Microbiol. 2015;33:248.

161. Datta P, Rani H, Chauhan R, Gombar S, Chander J. Healthcare-associated infections: Risk factors and epidemiology from an intensive care unit in Northern India. Indian J Anaesth. 2014;58:30.

162. Khanna V, Mukhopadhayay C, K E V, Verma M, Dabke P. Evaluation of central venous catheter associated blood stream infections: a microbiological observational study. J Pathog. 2013;2013:936864.

163. SD SR, Joseph MP, Lavi R, Macaden R. Infections Related to Vascular Catheters in a Pediatric Intensive Care Unit. INDIAN Pediatr. 2005;42:6.

164. Chakrabarti A, Sood P, Rudramurthy SM, Chen S, Kaur H, Capoor M, et al. Incidence, characteristics and outcome of ICU-acquired candidemia in India. Intensive Care Med. 2015;41:285-295.

165. Li J, Zhao QH, Huang KC, Li ZQ, Zhang LY, Qin DY, et al. Linezolid $v s$. vancomycin in treatment of methicillin-resistant Staphylococcus aureus infections: a meta-analysis. Eur Rev Med Pharmacol Sci. 2017;21:3974-3979.

166. Wilcox MH, Tack KJ, Bouza E, Herr DL, Ruf BR, Ijzerman MM, et al. Complicated Skin and Skin-Structure Infections and Catheter-Related Bloodstream Infections: Noninferiority of Linezolid in a Phase 3 Study. Clin Infect Dis. 2009;48: 203-212.

167. Yoon YK, Park DW, Sohn JW, Kim HY, Kim YS, Lee CS, et al. Multicenter Prospective Observational Study of the Comparative Efficacy and Safety of Vancomycin versus Teicoplanin in Patients with Health Care-Associated Methicillin-Resistant Staphylococcus aureus Bacteremia. Antimicrob Agents Chemother. 2014;58:317-324.

168. Raad I, Bompart F, Hachem R. Prospective, randomized dose-ranging open phase II pilot study of quinupristin/ dalfopristin versus vancomycin in the treatment of catheterrelated staphylococcal bacteremia. Eur J Clin Microbiol Infect Dis Off Publ Eur Soc Clin Microbiol. 1999;18:199-202.
169. Fowler VG, Boucher HW, Corey GR, Abrutyn E, Karchmer AW, Rupp ME, et al. Daptomycin versus standard therapy for bacteremia and endocarditis caused by Staphylococcus aureus. N Engl J Med. 2006;355:653-665.

170. Raad I, Darouiche R, Vazquez J, Lentnek A, Hachem R, Hanna $\mathrm{H}$, et al. Efficacy and Safety of Weekly Dalbavancin Therapy for Catheter-Related Bloodstream Infection Caused by GramPositive Pathogens. Clin Infect Dis. 2005;40:374-380.

171. Chong YP, Lee SO, Song EH, Lee EJ, Jang EY, Kim SH, et al. Quinupristin-dalfopristin versus linezolid for the treatment of vancomycin-resistant Enterococcus faecium bacteraemia: efficacy and development of resistance. Scand J Infect Dis. 2010;42:491-499.

172. Mora-Duarte J, Betts R, Rotstein C, Colombo AL, ThompsonMoya L, Smietana J, et al. Comparison of Caspofungin and Amphotericin B for Invasive Candidiasis. N Engl J Med. 2002;347:2020-2029.

173. Rex JH, Bennett JE, Sugar AM, Pappas PG, van der Horst CM, Edwards JE, et al. A randomized trial comparing fluconazole with amphotericin B for the treatment of candidemia in patients without neutropenia. Candidemia Study Group and the National Institute. N Engl J Med. 1994;331:1325-1330.

174. Havey TC, Fowler RA, Daneman N. Duration of antibiotic therapy for bacteremia: a systematic review and metaanalysis. Crit Care. 2011;15:R267.

175. Hooton TM, Bradley SF, Cardenas DD, Colgan R, Geerlings $\mathrm{SE}$, Rice JC, et al. Diagnosis, prevention, and treatment of catheter-associated urinary tract infection in adults: 2009 International Clinical Practice Guidelines from the Infectious Diseases Society of America. Clin Infect Dis Off Publ Infect Dis Soc Am. 2010;50:625-663.

176. Dudeck MA, Horan TC, Peterson KD, Allen-Bridson K, Morrell G, Pollock DA, et al. National Healthcare Safety Network (NHSN) Report, data summary for 2010, deviceassociated module. Am J Infect Control. 2011;39:798-816.

177. Centers for Disease Control. Urinary Tract Infection (Catheter-Associated Urinary Tract Infection [CAUTI] and Non-Catheter-Associated Urinary Tract Infection [UTI]) and Other Urinary System Infection [USI]) Events. Device-Assoc Module UTI. 2018;17.

178. Laupland KB, Bagshaw SM, Gregson DB, Kirkpatrick AW, Ross T, Church DL. Intensive care unit-acquired urinary tract infections in a regional critical care system. Crit Care Lond Engl. 2005;9:R60-65.

179. Rosser CJ, Bare RL, Meredith JW. Urinary tract infections in the critically ill patient with a urinary catheter. Am J Surg. 1999;177:287-90.

180. van der Kooi TII, de Boer AS, Manniën J, Wille JC, Beaumont MT, Mooi BW, et al. Incidence and risk factors of deviceassociated infections and associated mortality at the intensive care in the Dutch surveillance system. Intensive Care Med. 2007;33:271-8.

181. Alvarez-Lerma F, Palomar M, Olaechea P, Otal JJ, Insausti J, Cerdá E. [National Study of Control of Nosocomial Infection in Intensive Care Units. Evolutive report of the years 20032005]. Med Intensiva. 2007;31:6-17.

182. Chant C, Smith OM, Marshall JC, Friedrich JO. Relationship of catheter-associated urinary tract infection to mortality and length of stay in critically ill patients: a systematic review and meta-analysis of observational studies. Crit Care Med. 2011;39:1167-1173.

183. Laupland KB, Zygun DA, Davies HD, Church DL, Louie TJ, Doig CJ. Incidence and risk factors for acquiring nosocomial 
urinary tract infection in the critically ill. J Crit Care. 2002;17:50-57.

184. Tay MKX, Lee JYC, Wee IYJ, Oh HML. Evaluation of intensive care unit-acquired urinary tract infections in Singapore. Ann Acad Med Singapore. 2010;39:460-465.

185. Xie D, Fu X, Wang H, Wang L, Li R, Luo Q, et al. Annual point-prevalence of healthcare-associated infection surveys in a university hospital in China, 2007-2011. J Infect Public Health. 2013;6:416-422.

186. Leone M, Albanèse J, Garnier F, Sapin C, Barrau K, Bimar M-C, et al. Risk factors of nosocomial catheter-associated urinary tract infection in a polyvalent intensive care unit. Intensive Care Med. 2003;29:929-932.

187. Parlak E, Erol S, Kizilkaya M, Altoparlak U, Parlak M. [Nosocomial urinary tract infections in the intensive care unit patients]. Mikrobiyol Bul. 2007;41:39-49.

188. Karlowsky JA, Lagacé-Wiens PRS, Simner PJ, DeCorby MR, Adam HJ, Walkty A, et al. Antimicrobial resistance in urinary tract pathogens in Canada from 2007 to 2009: CANWARD surveillance study. Antimicrob Agents Chemother. 2011;55:3169-3175.

189. Temiz E, Piskin N, Aydemir H, Oztoprak N, Akduman D, Celebi G, et al. Factors associated with catheter-associated urinary tract infections and the effects of other concomitant nosocomial infections in intensive care units. Scand J Infect Dis. 2012;44:344-349.

190. Lewis SS, Knelson LP, Moehring RW, Chen LF, Sexton DJ, Anderson DJ. Comparison of non-intensive care unit (ICU) versus ICU rates of catheter-associated urinary tract infection in community hospitals. Infect Control Hosp Epidemiol. 2013;34:744-747.

191. Alvarez-Lerma F, Gracia-Arnillas MP, Palomar M, Olaechea P, Insausti J, López-Pueyo MJ, et al. Urethral catheter-related urinary infection in critical patients admitted to the ICU. Descriptive data of the ENVIN-UCI study. Med Intensiva. 2013;37:75-82.

192. Agarwal R, Gupta D, Ray P, Aggarwal AN, Jindal SK. Epidemiology, risk factors and outcome of nosocomial infections in a Respiratory Intensive Care Unit in North India. J Infect. 2006;53:98-105.

193. Habibi S, Wig N, Agarwal S, Sharma SK, Lodha R, Pandey $\mathrm{RM}$, et al. Epidemiology of nosocomial infections in medicine intensive care unit at a tertiary care hospital in northern India. Trop Doct. 2008;38:233-235.

194. Dasgupta S, Das S, Chawan NS, Hazra A. Nosocomial infections in the intensive care unit: Incidence, risk factors, outcome and associated pathogens in a public tertiary teaching hospital of Eastern India. Indian J Crit Care Med Peer-Rev Off Publ Indian Soc Crit Care Med. 2015;19:14-20.

195. Sahu MK, Siddharth B, Choudhury A, Vishnubhatla S, Singh $\mathrm{SP}$, Menon R, et al. Incidence, microbiological profile of nosocomial infections, and their antibiotic resistance patterns in a high volume Cardiac Surgical Intensive Care Unit. Ann Card Anaesth. 2016;19:281-287.

196. Vardakas KZ, Tansarli GS, Rafailidis PI, Falagas ME. Carbapenems versus alternative antibiotics for the treatment of bacteraemia due to Enterobacteriaceae producing extendedspectrum $\beta$-lactamases: a systematic review and metaanalysis. J Antimicrob Chemother. 2012;67:2793-803.

197. Savas L, Guvel S, Onlen Y, Savas N, Duran N. Nosocomial urinary tract infections: micro-organisms, antibiotic sensitivities and risk factors. West Indian Med J. 2006;55:188-193.

198. Yoon BI, Kim HS, Kim SD, Cho KJ, Kim SW, Ha U-S, et al. Changes in bacterial species and antibiotic sensitivity in intensive care unit: acquired urinary tract infection during 10 years interval (2001-2011). Urol J. 2014;11:1478-1484.

199. Karlowsky JA, Hoban DJ, Hackel MA, Lob SH, Sahm DF. Resistance among Gram-negative ESKAPE pathogens isolated from hospitalized patients with intra-abdominal and urinary tract infections in Latin American countries: SMART 2013-2015. Braz J Infect Dis Off Publ Braz Soc Infect Dis. 2017;21:343-348.

200. Seo YB, Lee J, Kim YK, Lee SS, Lee J-A, Kim HY, et al. Randomized controlled trial of piperacillin-tazobactam, cefepime and ertapenem for the treatment of urinary tract infection caused by extended-spectrum beta-lactamase-producing Escherichia coli. BMC Infect Dis. 2017;17:404.

201. Maraki S, Samonis G, Rafailidis PI, Vouloumanou EK, Mavromanolakis E, Falagas ME. Susceptibility of Urinary Tract Bacteria to Fosfomycin. Antimicrob Agents Chemother. 2009;53:4508-10.

202. Patel B, Patel K, Shetty A, Soman R, Rodrigues C. Fosfomycin Susceptibility in Urinary Tract Enterobacteriaceae. J Assoc Physicians India. 2017;65:14-16.

203. Johnson JR, Russo TA. Acute Pyelonephritis in Adults. N Engl J Med. 2018;378:48-59.

204. Dinh A, Toumi A, Blanc C, Descatha A, Bouchand F, Salomon J, et al. Management of febrile urinary tract infection among spinal cord injured patients. BMC Infect Dis. 2016;16:156.

205. Osawa K, Shigemura K, Yoshida H, Fujisawa M, Arakawa S. Candida urinary tract infection and Candida species susceptibilities to antifungal agents. J Antibiot (Tokyo). 2013;66:651-654.

206. World Health Organization. The Treatment of Diarrhoea. A Manual of Physicians and Other Senior Health Workers. Geneva: World Health Organization; 1990.

207. McFarland LV. Epidemiology of infectious and iatrogenic nosocomial diarrhea in a cohort of general medicine patients. Am J Infect Control. 1995;23:295-305.

208. PolageCR,SolnickJV,CohenSH.NosocomialDiarrhea:Evaluation and Treatment of Causes Other Than Clostridium difficile. Clin Infect Dis Off Publ Infect Dis Soc Am. 2012;55:982-989.

209. Thibault R, Graf S, Clerc A, Delieuvin N, Heidegger CP, Pichard C. Diarrhoea in the ICU: respective contribution of feeding and antibiotics. Crit Care. 2013;17:R153.

210. Elpern EH, Stutz L, Peterson S, Gurka DP, Skipper A. Outcomes associated with enteral tube feedings in a medical intensive care unit. Am J Crit Care Off Publ Am Assoc CritCare Nurses. 2004;13:221-7.

211. Wiesen P, Van Gossum A, Preiser J-C. Diarrhoea in the critically ill. Curr Opin Crit Care. 2006;12:149-54.

212. Bouza E, Muñoz P, Alonso R. Clinical manifestations, treatment and control of infections caused by Clostridium difficile. Clin Microbiol Infect. 2005;11:57-64.

213. Bobo LD, Dubberke ER. Recognition and Prevention of Hospital-Associated Enteric Infections in the Intensive Care Unit. Crit Care Med. 2010;38:S324-34.

214. Surawicz CM, Brandt LJ, Binion DG, Ananthakrishnan AN, Curry SR, Gilligan PH, et al. Guidelines for diagnosis, treatment, and prevention of Clostridium difficile infections. Am J Gastroenterol. 2013;108:478-98; quiz 499.

215. Planche T, Wilcox M. Reference assays for Clostridium difficile infection: one or two gold standards? J Clin Pathol. 2011; 64:1-5.

216. Surawicz CM, Brandt LJ, Binion DG, Ananthakrishnan AN, Curry SR, Gilligan PH, et al. Guidelines for diagnosis, 
treatment, and prevention of Clostridium difficile infections. Am J Gastroenterol. 2013;108:478-498.

217. Tirlapur N, Puthucheary ZA, Cooper JA, Sanders J, Coen PG, Moonesinghe SR, et al. Diarrhoea in the critically ill is common, associated with poor outcome, and rarely due to Clostridium difficile. Sci Rep. 2016;6:24691.

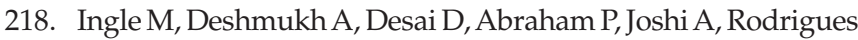
$\mathrm{C}$, et al. Prevalence and clinical course of Clostridium difficile infection in a tertiary-care hospital: a retrospective analysis. Indian J Gastroenterol Off J Indian Soc Gastroenterol. 2011;30:89-93.

219. Vishwanath S, Singhal A, D'Souza A, Mukhopadhyay C, Varma M, Bairy I. Clostridium difficile infection at a tertiary care hospital in south India. J Assoc Physicians India. 2013;61:804-806.

220. Marcon AP, Gamba MA, Vianna LAC. Nosocomial diarrhea in the intensive care unit. Braz J Infect Dis Off Publ Braz Soc Infect Dis. 2006;10:384-389.

221. Guallar C, Ariza J, Dominguez MA, Peña C, Grau I, Verdaguer $\mathrm{R}$, et al. An insidious nosocomial outbreak due to Salmonella enteritidis. Infect Control Hosp Epidemiol. 2004;25:10-15.

222. Johnston CP, Qiu H, Ticehurst JR, Dickson C, Rosenbaum P, Lawson $\mathrm{P}$, et al. Outbreak management and implications of a nosocomial norovirus outbreak. Clin Infect Dis Off Publ Infect Dis Soc Am. 2007;45:534=540.

223. Vasa CV, Glatt AE. Effectiveness and appropriateness of empiric metronidazole for Clostridium difficile-associated diarrhea. Am J Gastroenterol. 2003;98:354-358.

224. Minson Q, Mok S. Relationship between Antibiotic Exposure and Subsequent Clostridium difficile-Associated Diarrhea. Hosp Pharm. 2007;42:430-434.

225. Muto CA, Pokrywka M, Shutt K, Mendelsohn AB, Nouri $\mathrm{K}$, Posey K, et al. A large outbreak of Clostridium difficileassociated disease with an unexpected proportion of deaths and colectomies at a teaching hospital following increased fluoroquinolone use. Infect Control Hosp Epidemiol. 2005;26:273-280.

226. Polgreen PM, Chen YY, Cavanaugh JE, Ward M, Coffman $\mathrm{S}$, Hornick DB, et al. An outbreak of severe Clostridium difficile-associated disease possibly related to inappropriate antimicrobial therapy for community-acquired pneumonia. Infect Control Hosp Epidemiol. 2007;28:212-214.

227. Sundram F, Guyot A, Carboo I, Green S, Lilaonitkul M, Scourfield A. Clostridium difficile ribotypes 027 and 106: clinical outcomes and risk factors. J Hosp Infect. 2009;72:111-118.

228. Baxter R, Ray GT, Fireman BH. Case-control study of antibiotic use and subsequent Clostridium difficile-associated diarrhea in hospitalized patients. Infect Control Hosp Epidemiol. 2008;29:44-50.

229. Hensgens MPM, Goorhuis A, Dekkers OM, Kuijper EJ. Time interval of increased risk for Clostridium difficile infection after exposure to antibiotics. J Antimicrob Chemother. 2012;67:742-748.

230. Kallen AJ, Thompson A, Ristaino P, Chapman L, Nicholson A, Sim $\mathrm{B}-\mathrm{T}$, et al. Complete restriction of fluoroquinolone use to control an outbreak of Clostridium difficile infection at a community hospital. Infect Control Hosp Epidemiol. 2009;30:264-272.

231. Loo VG, Poirier L, Miller MA, Oughton M, Libman MD, Michaud S, et al. A predominantly clonal multi-institutional outbreak of Clostridium difficile-associated diarrhea with high morbidity and mortality. N Engl J Med. 2005;353:2442-2449.

232. McCusker ME, Harris AD, Perencevich E, Roghmann M-C. Fluoroquinolone Use and Clostridium difficile-Associated Diarrhea. Emerg Infect Dis. 2003;9:730-733.
233. Slimings C, Riley TV. Antibiotics and hospital-acquired Clostridium difficile infection: update of systematic review and meta-analysis. J Antimicrob Chemother. 2014;69:881-891.

234. Thomas C, Stevenson M, Williamson DJ, Riley TV. Clostridium difficile-Associated Diarrhea: Epidemiological Data from Western Australia Associated with a Modified Antibiotic Policy. Clin Infect Dis. 2002;35:1457-1462.

235. Musa SA, Robertshaw H, Thomson SJ, Cowan ML, Rahman TM. Clostridium difficile-associated disease acquired in the neurocritical care unit. Neurocrit Care. 2010;13:87-92.

236. Tripathy S, Nair P, Rothburn M. Clostridium difficile Associated Disease in a Neurointensive Care Unit. Front Neurol [Internet]. 2013 [cited 2018 Jun 7];4. Available from:

237. Alvarez-Lerma F, Palomar M, Villasboa A, Amador J, Almirall J, Posada MP, et al. Epidemiological study of Clostridium difficile infection in critical patients admitted to the Intensive Care Unit. Med Intensiva. 2014;38:558-566.

238. Ingle M, Deshmukh A, Desai D, Abraham P, Joshi A, Gupta $\mathrm{T}$, et al. Clostridium difficile as a cause of acute diarrhea: a prospective study in a tertiary care center. Indian J Gastroenterol Off J Indian Soc Gastroenterol. 2013;32: 179-183.

239. Ibrahim EH, Mehringer L, Prentice D, Sherman G, Schaiff R, Fraser $\mathrm{V}$, et al. Early versus late enteral feeding of mechanically ventilated patients: results of a clinical trial. JPEN J Parenter Enteral Nutr. 2002;26:174-181.

240. Lawrence SJ, Puzniak LA, Shadel BN, Gillespie KN, Kollef $\mathrm{MH}$, Mundy LM. Clostridium difficile in the Intensive Care Unit: Epidemiology, Costs, and Colonization Pressure. Infect Control Hosp Epidemiol. 2007;28:123-130.

241. Lübbert C, Johann C, Kekulé AS, Worlitzsch D, Weis S, Mössner J, et al. [Immunosuppressive treatment as a risk factor for the occurrence of Clostridium difficile infection (CDI)]. Z Gastroenterol. 2013;51:1251-1258.

242. Wang X, Cai L, Yu R, Huang W, Zong Z. ICU-Onset Clostridium difficile Infection in a University Hospital in China: A Prospective Cohort Study. McDowell A, editor. PLoS ONE. 2014;9:e111735.

243. Kaneria MV, Paul S. Incidence of Clostridium difficile associated diarrhoea in a tertiary care hospital. J Assoc Physicians India. 2012;60:26-28.

244. Buendgens L, Bruensing J, Matthes M, Dückers H, Luedde T, Trautwein C, et al. Administration of proton pump inhibitors in critically ill medical patients is associated with increased risk of developing Clostridium difficile-associated diarrhea. J Crit Care. 2014;29:696.e11-15.

245. Barletta JF, Sclar DA. Proton pump inhibitors increase the risk for hospital-acquired Clostridium difficile infection in critically ill patients. Crit Care Lond Engl. 2014;18:714.

246. Nelson RL, Suda KJ, Evans CT. Antibiotic treatment for Clostridium difficile-associated diarrhoea in adults. Cochrane Database Syst Rev. 2017;3:CD004610.

247. Johnson S, Louie TJ, Gerding DN, Cornely OA, Chasan-Taber $\mathrm{S}$, Fitts D, et al. Vancomycin, metronidazole, or tolevamer for Clostridium difficile infection: results from two multinational, randomized, controlled trials. Clin Infect Dis Off Publ Infect Dis Soc Am. 2014;59:345-354.

248. Zar FA, Bakkanagari SR, Moorthi KMLST, Davis MB. A comparison of vancomycin and metronidazole for the treatment of Clostridium difficile-associated diarrhea, stratified by disease severity. Clin Infect Dis Off Publ Infect Dis Soc Am. 2007;45:302-307. 
249. Teasley DG, Gerding DN, Olson MM, Peterson LR, Gebhard RL, Schwartz MJ, et al. Prospective randomised trial of metronidazole versus vancomycin for Clostridium-difficileassociated diarrhoea and colitis. Lancet Lond Engl. 1983;2:10431046.

250. Wenisch C, Parschalk B, Hasenhündl M, Hirschl AM, Graninger W. Comparison of vancomycin, teicoplanin, metronidazole, and fusidic acid for the treatment of Clostridium difficile-associated diarrhea. Clin Infect Dis Off Publ Infect Dis Soc Am. 1996;22:813-818.

251. Louie TJ, Miller MA, Mullane KM, Weiss K, Lentnek A, Golan $\mathrm{Y}$, et al. Fidaxomicin versus vancomycin for Clostridium difficile infection. N Engl J Med. 2011;364:422-431.

252. Mullane KM, Miller MA, Weiss K, Lentnek A, Golan Y, Sears PS, et al. Efficacy of fidaxomicin versus vancomycin as therapy for Clostridium difficile infection in individuals taking concomitant antibiotics for other concurrent infections. Clin Infect Dis Off Publ Infect Dis Soc Am. 2011;53:440-447.

253. Cornely OA, Nathwani D, Ivanescu C, Odufowora-Sita $\mathrm{O}$, Retsa P, Odeyemi IAO. Clinical efficacy of fidaxomicin compared with vancomycin and metronidazole in Clostridium difficile infections: a meta-analysis and indirect treatment comparison. J Antimicrob Chemother. 2014;69:2892-2900.

254. de Lalla F, Nicolin R, Rinaldi E, Scarpellini P, Rigoli R, Manfrin $\mathrm{V}$, et al. Prospective study of oral teicoplanin versus oral vancomycin for therapy of pseudomembranous colitis and Clostridium difficile-associated diarrhea. Antimicrob Agents Chemother. 1992;36:2192-2196.

255. Wenisch C, Paschalk B, Hasenhundi M, Hirschi A, Graninger W. Comparison of vancomycin, teicoplanin, metronidazole, and fusidic acid for the treatment of Clostridium difficile-associated diarrhea. - PubMed - NCBI [Internet]. [cited 2018 Jun 8].

256. Drekonja DM, Amundson WH, Decarolis DD, Kuskowski MA, Lederle FA, Johnson JR. Antimicrobial use and risk for recurrent Clostridium difficile infection. Am J Med. 2011;124:1081 e1-7.

257. McFarland LV, Surawicz CM, Greenberg RN, Fekety R, Elmer GW, Moyer KA, et al. A randomized placebocontrolled trial of Saccharomyces boulardii in combination with standard antibiotics for Clostridium difficile disease. JAMA. 1994;271:19131918.

258. Pillai A, Nelson R. Probiotics for treatment of Clostridium difficile-associated colitis in adults. Cochrane Database Syst Rev. 2008;CD004611.

259. Lau CS, Chamberlain RS. Probiotics are effective at preventing Clostridium difficile-associated diarrhea: a systematic review and meta-analysis. Int J Gen Med. 2016;9:27-37.

260. O'Horo JC, Jindai K, Kunzer B, Safdar N. Treatment of recurrent Clostridium difficile infection: a systematic review. Infection. 2014;42:43-59.

261. McFarland LV, Elmer GW, Surawicz CM. Breaking the cycle: treatment strategies for 163 cases of recurrent Clostridium difficile disease. Am J Gastroenterol. 2002;97:1769-1775.

262. Pépin J, Routhier S, Gagnon S, Brazeau I. Management and outcomes of a first recurrence of Clostridium difficile-associated disease in Quebec, Canada. Clin Infect Dis Off Publ Infect Dis Soc Am. 2006;42:758-764.

263. Surawicz CM, McFarland LV, Greenberg RN, Rubin M, Fekety R, Mulligan ME, et al. The search for a better treatment for recurrent Clostridium difficile disease: use of high-dose vancomycin combined with Saccharomyces boulardii. Clin Infect Dis Off Publ Infect Dis Soc Am. 2000;31:1012-1017.
264. Cornely OA, Miller MA, Louie TJ, Crook DW, Gorbach SL. Treatment of first recurrence of Clostridium difficile infection: fidaxomicin versus vancomycin. Clin Infect Dis Off Publ Infect Dis Soc Am. 2012;55 Suppl 2:S154-161.

265. Friedman-Korn T, Livovsky DM, Maharshak N, Aviv Cohen N, Paz K, Bar-Gil Shitrit A, et al. Fecal Transplantation for Treatment of Clostridium difficile Infection in Elderly and Debilitated Patients. Dig Dis Sci. 2018;63:198-203.

266. Banks PA, Bollen TL, Dervenis C, Gooszen HG, Johnson CD, Sarr MG, et al. Classification of acute pancreatitis-2012: revision of the Atlanta classification and definitions by international consensus. Gut. 2013;62:102-111.

267. Yadav D, Lowenfels AB. Trends in the epidemiology of the first attack of acute pancreatitis: a systematic review. Pancreas. 2006;33:323-30.

268. CotéGA, Yadav D, Slivka A, Hawes RH, Anderson MA, Burton FR et al. Alcohol and smoking as risk factors in an epidemiology study of patients with chronic pancreatitis. Clin Gastroenterol Hepatol Off Clin Pract J Am Gastroenterol Assoc. 2011;9:266-273; quiz e27.

269. Marshall JC, Cook DJ, Christou NV, Bernard GR, Sprung CL, Sibbald WJ. Multiple organ dysfunction score: a reliable descriptor of a complex clinical outcome. Crit Care Med. 1995;23:1638-1652.

270. de-Madaria E, Pamies-Guilabert J, García G, Benito JL de, Hinojosa-Guadix J, Álamo FFG del, et al. Su1890 Local and Systemic Complications of Acute Pancreatitis According to the Revised Atlanta Classification: A Nation-Wide Multicenter Prospective Study. Gastroenterology. 2015;148:S-545.

271. Banks PA, Bollen TL, Dervenis C, Gooszen HG, Johnson CD, Sarr MG, et al. Classification of acute pancreatitis--2012: revision of the Atlanta classification and definitions by international consensus. Gut. 2013;62:102-111.

272. Baron TH, Morgan DE. Acute necrotizing pancreatitis. N Engl J Med. 1999;340:1412-7.

273. Beger HG, Rau B, Mayer J, Pralle U. Natural course of acute pancreatitis. World J Surg. 1997;21:130-135.

274. Balthazar EJ, Robinson DL, Megibow AJ, Ranson JH. Acute pancreatitis: value of CT in establishing prognosis. Radiology. 1990;174:331-336.

275. Werge M, Novovic S, Schmidt PN, Gluud LL. Infection increases mortality in necrotizing pancreatitis: A systematic review and meta-analysis. Pancreatol Off J Int Assoc Pancreatol IAP Al. 2016;16:698-707.

276. Beger HG, Rau B, Isenmann R. Natural history of necrotizing pancreatitis. Pancreatol Off J Int Assoc Pancreatol IAP Al. 2003;3:93-101.

277. Tenner S, Baillie J, DeWitt J, Vege SS. American College of Gastroenterology Guideline: Management of Acute Pancreatitis. Am J Gastroenterol. 2013;108:1400-1415.

278. Yokoe M, Takada T, Mayumi T, Yoshida M, Isaji S, Wada K, et al Japanese guidelines for the management of acute pancreatitis: Japanese Guidelines 2015. J Hepato-Biliary-Pancreat Sci. 2015;22:405-432.

279. Garg PK, Khanna S, Bohidar NP, Kapil A, Tandon RK Incidence, spectrum and antibiotic sensitivity pattern of bacterial infections among patients with acute pancreatitis. J Gastroenterol Hepatol. 2001;16:1055-1059.

280. Dellinger EP, Tellado JM, Soto NE, Ashley SW, Barie PS, Dugernier T, et al. Early antibiotic treatment for severe acute necrotizing pancreatitis: a randomized, double-blind, placebocontrolled study. Ann Surg. 2007;245:674. 
281. Isenmann R, Rau B, Beger HG. Bacterial infection and extent of necrosis are determinants of organ failure in patients with acute necrotizing pancreatitis. BJS. 1999;86:1020-1024.

282. Büchler MW, Gloor B, Müller CA, Friess H, Seiler CA, Uhl W. Acute necrotizing pancreatitis: treatment strategy according to the status of infection. Ann Surg. 2000;232:619-626.

283. Mutinga M, Rosenbluth A, Tenner SM, Odze RR, Sica GT, Banks PA. Does mortality occur early or late in acute pancreatitis? Int J Pancreatol. 2000;28:91-95.

284. Mann DV, Hershman MJ, Hittinger R, Glazer G. Multicentre audit of death from acute pancreatitis. BJS. 1994;81:890-893.

285. Padhan RK, Jain S, Agarwal S, Harikrishnan S, Vadiraja P, Behera S, et al. Primary and Secondary Organ Failures Cause Mortality Differentially in Acute Pancreatitis and Should be Distinguished. Pancreas. 2018;47:302-307.

286. Gerzof SG, Banks PA, Robbins AH, Johnson WC, Spechler SJ, Wetzner SM, et al. Early diagnosis of pancreatic infection by computed tomography-guided aspiration. Gastroenterology. 1987;93:1315-1320.

287. Noor MT, Radhakrishna Y, Kochhar R, Ray P, Wig JD, Sinha $\mathrm{SK}$, et al. Bacteriology of infection in severe acute pancreatitis. JOP J Pancreas. 2011;12:19-25.

288. Runkel N. Bacterial Translocation in Acute Pancreatitis. Dig Surg. 1996;13:269-272.

289. Moody FG, Haley-Russell D, Muncy DM. Intestinal transit and bacterial translocation in obstructive pancreatitis. Dig Dis Sci. 1995;40:1798-1804.

290. Jain S, Mahapatra SJ, Gupta S, Shalimar, Garg PK. Infected Pancreatic Necrosis due to Multidrug-Resistant Organisms and Persistent Organ failure Predict Mortality in Acute Pancreatitis. Clin Transl Gastroenterol. 2018;9:190.

291. Bassi C, Falconi M, Girelli R, Nifosi F, Elio A, Martini N, et al. Microbiological findings in severe pancreatitis. Surg Res Commun. 1989;5:1-4.

292. Widdison AL, Karanjia ND. Pancreatic infection complicating acute pancreatitis. Br J Surg. 1993;80:148-154.

293. Talukdar R, Nechutova H, Clemens M, Vege SS. Could rising BUN predict the future development of infected pancreatic necrosis? Pancreatol Off J Int Assoc Pancreatol IAP Al. 2013;13: 355-359.

294. Howard TJ, Temple MB. Prophylactic antibiotics alter the bacteriology of infected necrosis in severe acute pancreatitis. J Am Coll Surg. 2002;195:759-67.

295. Ji L, Lv J-C, Song Z-F, Jiang M-T, Li L, Sun B. Risk factors of infected pancreatic necrosis secondary to severe acute pancreatitis. Hepatobiliary Pancreat Dis Int. 2016;15:428-433.

296. Isenmann R, Rünzi M, Kron M, Kahl S, Kraus D, Jung N, et al. Prophylactic antibiotic treatment in patients with predicted severe acute pancreatitis: a placebo-controlled, double-blind trial1. Gastroenterology. 2004;126:997-1004.

297. Lim CLL, Lee W, Liew YX, Tang SSL, Chlebicki MP, Kwa AL-H. Role of antibiotic prophylaxis in necrotizing pancreatitis: a meta-analysis. J Gastrointest Surg. 2015;19:480-491.

298. Bassi C, Pederzoli P, Vesentini S, Falconi M, Bonora A, Abbas $\mathrm{H}$, et al. Behavior of antibiotics during human necrotizing pancreatitis. Antimicrob Agents Chemother. 1994;38: 830-836.

299. Büchler M, Malfertheiner P, Frie $\beta$ H, Isenmann R, Vanek E, Grimm $\mathrm{H}$, et al. Human pancreatic tissue concentration of bactericidal antibiotics. Gastroenterology. 1992;103:1902-1908.

300. Ibrahim N, Chan L, Li Ling T, Mat Nor MB. Guide to Antimicrobial Therapy 2017. 2017.
301. Runzi M, Niebel W, Goebell H, Gerken G, Layer P. Severe acute pancreatitis: nonsurgical treatment of infected necroses. Pancreas. 2005;30:195-199.

302. Hartwig W, Maksan S-M, Foitzik T, Schmidt J, Herfarth C, Klar E. Reduction in mortality with delayed surgical therapy of severe pancreatitis. J Gastrointest Surg. 2002;6:481-487.

303. Garg PK, Sharma M, Madan K, Sahni P, Banerjee D, Goyal R. Primary conservative treatment results in mortality comparable to surgery in patients with infected pancreatic necrosis. Clin Gastroenterol Hepatol. 2010;8:1089-1094.

304. Mouli VP, Sreenivas V, Garg PK. Efficacy of conservative treatment, without necrosectomy, for infected pancreatic necrosis: a systematic review and meta-analysis. Gastroenterology. 2013;144:333-340.

305. Adler DG, Chari ST, Dahl TJ, Farnell MB, Pearson RK. Conservative management of infected necrosis complicating severe acute pancreatitis. Am J Gastroenterol. 2003;98:98.

306. Leung JW, Ling TK, Chan RC, Cheung SW, Lai CW, Sung JJ, et al. Antibiotics, biliary sepsis, and bile duct stones. Gastrointest Endosc. 1994;40:716-721.

307. Kiriyama S, Takada T, Strasberg SM, Solomkin JS, Mayumi T, Pitt HA, et al. New diagnostic criteria and severity assessment of acute cholangitis in revised Tokyo Guidelines. J HepatoBiliary-Pancreat Sci. 2012;19:548-556.

308. Williams EJ, Green J, Beckingham I, Parks R, Martin D, Lombard M. Guidelines on the management of common bile duct stones (CBDS). Gut. 2008;57:1004-1021.

309. Friedman GD. Natural history of asymptomatic and symptomatic gallstones. Am J Surg. 1993;165:399-404.

310. McSherry CK, Ferstenberg H, Calhoun WF, Lahman E, Virshup $\mathrm{M}$. The natural history of diagnosed gallstone disease in symptomatic and asymptomatic patients. Ann Surg. 1985;202:59.

311. Andriulli A, Loperfido S, Napolitano G, Niro G, Valvano MR, Spirito $\mathrm{F}$, et al. Incidence rates of post-ERCP complications: a systematic survey of prospective studies. Am J Gastroenterol. 2007;102:1781.

312. Anderson DJ, Shimpi RA, McDonald JR, Branch MS, Kanafani ZA, Harger J, et al. Infectious complications following endoscopic retrograde cholangiopancreatography: an automated surveillance system for detecting postprocedure bacteremia. Am J Infect Control. 2008;36:592-594.

313. Lipsett PA, Pitt HA. Acute cholangitis. Surg Clin North Am. 1990;70:1297-1312.

314. Gigot JF, Leese T, Dereme T, Coutinho J, Castaing D, Bismuth H. Acute cholangitis. Multivariate analysis of risk factors. Ann Surg. 1989;209:435.

315. Melzer M, Toner R, Lacey S, Bettany E, Rait G. Biliary tract infection and bacteraemia: presentation, structural abnormalities, causative organisms and clinical outcomes. Postgrad Med J. 2007;83:773-776.

316. Saharia PC, Zuidema GD, Cameron JL. Primary common duct stones. Ann Surg. 1977;185:598.

317. Sung YK, Lee JK, Lee KH, Lee KT, Kang C-I. The clinical epidemiology and outcomes of bacteremic biliary tract infections caused by antimicrobial-resistant pathogens. Am J Gastroenterol. 2012;107:473.

318. Bapat RD, Supe AN, Patwardhan A, Kocher HM, Parab S, Sathe MJ. Biliary sepsis: an ascending infection. Indian J Gastroenterol Off J Indian Soc Gastroenterol. 1996;15:126-128.

319. Shenoy SM, Shenoy S, Gopal S, Tantry BV, Baliga S, Jain A. Clinicomicrobiological analysis of patients with cholangitis. Indian J Med Microbiol. 2014;32:157. 
320. Ortega M, Marco F, Soriano A, Almela M, Martinez JA, Lopez J, et al. Epidemiology and prognostic determinants of bacteraemic biliary tract infection. J Antimicrob Chemother. 2012;67:1508-1513.

321. Sahu MK, Chacko A, Dutta AK, Prakash JAJ. Microbial profile and antibiotic sensitivity pattern in acute bacterial cholangitis. Indian J Gastroenterol. 2011;30:204.

322. Englesbe MJ, Dawes LG. Resistant pathogens in biliary obstruction: importance of cultures to guide antibiotic therapy. Hpb. 2005;7:144-148.

323. Miura F, Okamoto K, Takada T, Strasberg SM, Asbun HJ, Pitt HA, et al. Tokyo Guidelines 2018: initial management of acute biliary infection and flowchart for acute cholangitis. J Hepato-Biliary-Pancreat Sci. 2018;25:31-40.

324. Tanaka A, Takada T, Kawarada Y, Nimura Y, Yoshida M, Miura F, et al. Antimicrobial therapy for acute cholangitis: Tokyo Guidelines. J Hepatobiliary Pancreat Surg. 2007;14:59-67.

325. Solomkin JS, Mazuski JE, Bradley JS, Rodvold KA, Goldstein EJ, Baron EJ, et al. Diagnosis and management of complicated intra-abdominal infection in adults and children: guidelines by the Surgical Infection Society and the Infectious Diseases Society of America. Clin Infect Dis. 2010;50:133-164.

326. Kaplan G, Gregson D, Laupland K. Population-based study of the epidemiology of and the risk factors for pyogenic liver abscess. Clin Gastroenterol Hepatol. 2004;2:1032-1038.

327. Chan K-S, Chen C-M, Cheng K-C, Hou C-C, Lin H-J, Yu W-L. Pyogenic liver abscess: a retrospective analysis of 107 patients during a 3-year period. Jpn J Infect Dis. 2005;58:366-368.

328. Tsai F-C, Huang Y-T, Chang L-Y, Wang J-T. Pyogenic Liver Abscess as Endemic Disease, Taiwan. Emerg Infect Dis. 2008;14:1592-1600.

329. Abbas MT, Khan FY, Muhsin SA, Al-Dehwe B, Abukamar M, Elzouki A-N. Epidemiology, Clinical Features and Outcome of Liver Abscess: A single Reference Center Experience in Qatar. Oman Med J. 2014;29:260-263.

330. Jain V, Manjavkar S, Kapur P, Durfishan ., Rajput D, Mir T. Clinical and biochemical profile of liver abscess patients. Int J Res Med Sci. 2017;5:2596.

331. Lok K-H, Li K-F, Li K-K, Szeto M-L. Pyogenic liver abscess: clinical profile, microbiological characteristics, and management in a Hong Kong hospital. J Microbiol Immunol Infect Wei Mian Yu Gan Ran Za Zhi. 2008;41:483-490.

332. Huang C-J, Pitt HA, Lipsett PA, Osterman FA, Lillemoe KD, Cameron JL, et al. Pyogenic Hepatic Abscess: Changing Trends Over 42 Years. Ann Surg. 1996;223:600-609.

333. Mohsen AH. Liver abscess in adults: ten years experience in a UK centre. QJM. 2002;95:797-802.

334. Ng FH, Wong WM, Wong BCY, Kng C, Wong SY, Lai KC, et al. Sequential intravenous/oral antibiotic vs. continuous intravenous antibiotic in the treatment of pyogenic liver abscess. Aliment Pharmacol Ther. 2002;16:1083-1090.

335. Rahimian J, Wilson T, Oram V, Holzman RS. Pyogenic Liver Abscess: Recent Trends in Etiology and Mortality. Clin Infect Dis. 2004;39:1654-1659.

336. Luo M, Yang X-X, Tan B, Zhou X-P, Xia H-M, Xue J, et al Distribution of common pathogens in patients with pyogenic liver abscess in China: a meta-analysis. Eur J Clin Microbiol Infect Dis. 2016:35:1557-1565.

337. Ghosh S, Sharma S, Gadpayle AK, Gupta HK, Mahajan RK, Sahoo R, et al. Clinical, Laboratory, and Management Profile in Patients of Liver Abscess from Northern India. J Trop Med. 2014;2014:1-8.

338. Poovorawan K, Pan-ngum W, Soonthornworasiri N, Kulrat C, Kittitrakul C, Wilairatana P, et al. Burden of Liver Abscess and Survival Risk Score in Thailand: A Population-Based Study. Am J Trop Med Hyg. 2016;95:683-688.

339. Peris J, Bellot P, Roig P, Reus S, Carrascosa S, González-Alcaide $\mathrm{G}$, et al. Clinical and epidemiological characteristics of pyogenic liver abscess in people 65 years or older versus people under 65: a retrospective study. BMC Geriatr. 2017;17:161.

340. Ghosh S, Sharma S, Gadpayle AK, Gupta HK, Mahajan RK, Sahoo R, et al. Clinical, laboratory, and management profile in patients of liver abscess from northern India. J Trop Med. 2014;2014:142382

341. Sharma MP, Rai RR, Acharya SK, Ray JC, Tandon BN. Needle aspiration of amoebic liver abscess. BMJ. 1989;299:1308-1309.

342. Lasserre R, Jaroonvesama N, Kurathong S, Soh C-T. SingleDay Drug Treatment of Amebic Liver Abscess. Am J Trop Med Hyg. 1983;32:723-726.

343. Bhatia S, Karnad DR, Oak JL. Randomized double-blind trial of metronidazole versus secnidazole in amebic liver abscess. Indian J Gastroenterol Off J Indian Soc Gastroenterol. 1998;17:53-54.

344. McGarr PL, Madiba TE, Thomson SR, Corr P. Amoebic liver abscess - results of a conservative management policy. 2003;93:5.

345. Cheng H-P, Siu LK, Chang F-Y. Extended-Spectrum Cephalosporin Compared to Cefazolin for Treatment of Klebsiella pneumoniae-Caused Liver Abscess. Antimicrob Agents Chemother. 2003;47:2088-2092.

346. Liu Y, Wang J, Jiang W. An Increasing Prominent Disease of Klebsiella pneumoniae Liver Abscess: Etiology, Diagnosis, and Treatment. Gastroenterol Res Pract. 2013;2013:1-12.

347. Lübbert C, Wiegand J, Karlas T. Therapy of Liver Abscesses. Viszeralmedizin. 2014;30:3-3.

348. Pal P, Ray S, Moulick A, Dey S, Jana A, Banerjee K. Liver abscess caused by Burkholderia pseudomallei in a young man: A case report and review of literature. World J Clin Cases WJCC. 2014;2:604.

349. Sentochnik DE, Eliopoulos GM, Ferraro MJ, Moellering RC, Jr. Comparative in vitro activity of SM7338, a new carbapenem antimicrobial agent. Antimicrob Agents Chemother. 1989;33:1232

350. Pastagia M, Arumugam V. Klebsiella pneumoniae liver abscesses in a public hospital in Queens, New York. Travel Med Infect Dis. 2008;6:228-233.

351. Yu SCH, Ho SSM, Lau WY, Yeung DTK, Yuen EHY, Lee PSF, et al. Treatment of pyogenic liver abscess: prospective randomized comparison of catheter drainage and needle aspiration. Hepatol Baltim Md. 2004;39:932-938.

352. Zerem E, Hadzic A. Sonographically guided percutaneous catheter drainage versus needle aspiration in the management of pyogenic liver abscess. AJR Am J Roentgenol. 2007;189:W138142.

353. Cai Y-L, Xiong X-Z, Lu J, Cheng Y, Yang C, Lin Y-X, et al. Percutaneous needle aspiration versus catheter drainage in the management of liver abscess: a systematic review and meta-analysis. HPB. 2015;17:195-201.

354. Heneghan HM, Healy NA, Martin ST, Ryan RS, Nolan N, Traynor $\mathrm{O}$, et al. Modern management of pyogenic hepatic abscess: a case series and review of the literature. BMC Res Notes. 2011;4:80 
355. Andreu M, Sola R, Sitges-Serra A, Alia C, Gallen M, Vila MC, et al. Risk factors for spontaneous bacterial peritonitis in cirrhotic patients with ascites. Gastroenterology. 1993;104:1133-1138.

356. Uncu N, Bülbül M, Yildiz N, Noyan A, Koşan C, Kavukçu S, et al. Primary peritonitis in children with nephrotic syndrome: results of a 5-year multicenter study. Eur J Pediatr. 2010;169:73-76.

357. Mishra SP, Tiwary SK, Mishra M, Gupta SK. An introduction of Tertiary Peritonitis. J Emerg Trauma Shock. 2014;7:121.

358. Weiss G, Steffanie W, Lippert H. [Peritonitis: main reason of severe sepsis in surgical intensive care]. Zentralbl Chir. 2007;132:130-137.

359. Calandra T, Cohen J, International Sepsis Forum Definition of Infection in the ICU Consensus Conference. The international sepsis forum consensus conference on definitions of infection in the intensive care unit. Crit Care Med. 2005;33:1538-1548.

360. Reemst PH, van Goor H, Goris RJ. SIRS, MODS and tertiary peritonitis. Eur J Surg Suppl Acta Chir Suppl. 1996;47-48; discussion 49.

361. Buijk SE, Bruining HA. Future directions in the management of tertiary peritonitis. Intensive Care Med. 2002;28:1024-1029.

362. Evans HL, Raymond DP, Pelletier SJ, Crabtree TD, Pruett TL, Sawyer RG. Diagnosis of intra-abdominal infection in the critically ill patient. Curr Opin Crit Care. 2001;7:117-121.

363. Ballus J, Lopez-Delgado JC, Sabater-Riera J, Perez-Fernandez XL, Betbese AJ, Roncal JA. Factors Associated with the Development of Tertiary Peritonitis in Critically Ill Patients. Surg Infect. 2017;18:588-595.

364. França A, Giordano HM, Sevá-Pereira T, Soares EC. Five days of ceftriaxone to treat spontaneous bacterial peritonitis in cirrhotic patients. J Gastroenterol. 2002;37:119-122.

365. Marshall JC, Innes M. Intensive care unit management of intra-abdominal infection. Crit Care Med. 2003;31:2228-2237.

366. Nathens AB, Rotstein OD, Marshall JC. Tertiary peritonitis: clinical features of a complex nosocomial infection. World J Surg. 1998;22:158-163.

367. Rimola A, Salmerón JM, Clemente G, Rodrigo L, Obrador A, Miranda ML, et al. Two different dosages of cefotaxime in the treatment of spontaneous bacterial peritonitis in cirrhosis: results of a prospective, randomized, multicenter study. Hepatol Baltim Md. 1995;21:674-679.

368. European Association for the Study of the Liver. EASL clinical practice guidelines on the management of ascites, spontaneous bacterial peritonitis, and hepatorenal syndrome in cirrhosis. J Hepatol. 2010;53:397-417.

369. Navasa M, Follo A, Llovet JM, Clemente G, Vargas V, Rimola A, et al. Randomized, comparative study of oral ofloxacin versus intravenous cefotaxime in spontaneous bacterial peritonitis. Gastroenterology. 1996;111:1011-1017.

370. Terg R, Cobas S, Fassio E, Landeira G, Ríos B, Vasen W, et al. Oral ciprofloxacin after a short course of intravenous ciprofloxacin in the treatment of spontaneous bacterial peritonitis: results of a multicenter, randomized study. J Hepatol. 2000;33:564-569.

371. Jindal A, Kumar M, Bhadoria AS, Maiwall R, Sarin SK. A randomized open label study of "imipenem vs. cefepime" in spontaneous bacterial peritonitis. Liver Int Off J Int Assoc Study Liver. 2016;36:677-687.

372. Brismar B, Malmborg AS, Tunevall G, Wretlind B, Bergman L, Mentzing LO, et al. Piperacillin-tazobactam versus imipenemcilastatin for treatment of intra-abdominal infections. Antimicrob Agents Chemother. 1992;36:2766-2773.
373. Montravers P, Lepape A, Dubreuil L, Gauzit R, Pean Y, Benchimol D, et al. Clinical and microbiological profiles of community-acquired and nosocomial intra-abdominal infections: results of the French prospective, observational EBIIA study. J Antimicrob Chemother. 2009;63:785-94.

374. Steinbach CL, Töpper C, Adam T, Kees MG. Spectrum adequacy of antibiotic regimens for secondary peritonitis: a retrospective analysis in intermediate and intensive care unit patients. Ann Clin Microbiol Antimicrob. 2015;14:48.

375. Sawyer RG, Claridge JA, Nathens AB, Rotstein OD, Duane TM, Evans HL, et al. Trial of Short-Course Antimicrobial Therapy for Intraabdominal Infection. N Engl J Med. 2015;372:1996-2005.

376. Thigpen MC, Whitney CG, Messonnier NE, Zell ER, Lynfield $\mathrm{R}$, Hadler JL, et al. Bacterial meningitis in the United States, 1998-2007. N Engl J Med. 2011;364:2016-2025.

377. Moon S-Y, Chung DR, Kim S-W, Chang HH, Lee H, Jung DS, et al. Changing etiology of community-acquired bacterial meningitis in adults: a nationwide multicenter study in Korea. Eur J Clin Microbiol Infect Dis. 2010;29:793-800.

378. Durand ML, Calderwood SB, Weber DJ, Miller SI, Southwick FS, Caviness VS, et al. Acute Bacterial Meningitis in Adults - A Review of 493 Episodes. N Engl J Med. 1993;328:21-28.

379. Faustini A, Arca' M, Fusco D, Perucci CA. Prognostic factors and determinants of fatal outcome due to bacterial meningitis in the Lazio region of Italy, 1996-2000. Int J Infect Dis. 2007;11:137-144.

380. Dzupova O, Rozsypal H, Prochazka B, Benes J. Acute bacterial meningitis in adults: Predictors of outcome. Scand J Infect Dis. 2009;41:348-354.

381. Glimaker M, Johansson B, Grindborg O, Bottai M, Lindquist L, Sjolin J. Adult Bacterial Meningitis: Earlier Treatment and Improved Outcome Following Guideline Revision Promoting Prompt Lumbar Puncture. Clin Infect Dis. 2015;60:1162-1169.

382. Grindborg ö., Naucler P, Sjölin J, Glimåker M. Adult bacterial meningitis—a quality registry study: earlier treatment and favourable outcome if initial management by infectious diseases physicians. Clin Microbiol Infect. 2015;21:560-566.

383. Armstrong D, Ashworth M, Dregan A, White P. The relationship between prior antimicrobial prescription and meningitis: a case-control study. Br J Gen Pract. 2016;66:e228-233.

384. Domingo P, Pomar V, de Benito N, Coll P. The spectrum of acute bacterial meningitis in elderly patients. BMC Infect Dis. 2013;13:108.

385. Tankhiwale S S, Jagtap P M, Khadse R K, Jalgaonkar S V. Bacteriological study of pyogenic meningitis with special reference to C-reactive protein. Indian J Med Microbiol. 2001;19:159.

386. Chandramuki A, Mani R, Pradhan S, Nagarathna S, Wasiulla R. Bacteriological profile of community acquired acute bacterial meningitis: A ten-year retrospective study in a tertiary neurocare centre in South India. Indian J Med Microbiol. 2007;25:108.

387. Madhumita P, Gupta N. Clinical and bacteriological spectrum of community-acquired acute bacterial meningitis in adults at a tertiary care hospital in northern India. Int J Nutr Pharmacol Neurol Dis. 2011;1:194.

388. Vibha D, Bhatia R, Prasad K, Srivastava MVP, Tripathi M, Singh MB. Clinical Features and Independent Prognostic Factors for Acute Bacterial Meningitis in Adults. Neurocrit Care. 2010;13:199-204. 
389. Williamson RA, Phillips-Bute BG, McDonagh DL, Gray MC, Zomorodi AR, Olson DM, et al. Predictors of extraventricular drain-associated bacterial ventriculitis. J Crit Care. 2014;29:77-82.

390. Chi H, Chang K-Y, Chang H-C, Chiu N-C, Huang F-Y. Infections associated with indwelling ventriculostomy catheters in a teaching hospital. Int J Infect Dis. 2010;14:e216-219.

391. Chen C-H, Chang C-Y, Lin L-J, Chen WL, Chang Y-J, Wang $\mathrm{S}-\mathrm{H}$, et al. Risk factors associated with postcraniotomy meningitis: A retrospective study. Medicine (Baltimore). 2016;95:e4329.

392. Murthy SB, Moradiya Y, Shah J, Hanley DF, Ziai WC. Incidence, Predictors, and Outcomes of Ventriculostomy-Associated Infections in Spontaneous Intracerebral Hemorrhage. Neurocrit Care. 2016;24:389-396.

393. Citerio G, Signorini L, Bronco A, Vargiolu A, Rota M, Latronico N. External Ventricular and Lumbar Drain Device Infections in ICU Patients: A Prospective Multicenter Italian Study. Crit Care Med. 2015;43:1630-1637.

394. Arabi Y, Memish ZA, Balkhy HH, Francis C, Ferayan A, Al Shimemeri A, et al. Ventriculostomy-associated infections: Incidence and risk factors. Am J Infect Control. 2005;33:137-143.

395. Beer R, Lackner P, Pfausler B, Schmutzhard E. Nosocomial ventriculitis and meningitis in neurocritical care patients. J Neurol. 2008;255:1617-1624.

396. Korinek A-M, Korinek A-M, Laisne M-J, Achou C, Bromberg N, Dagreou F, et al. Risk Factors for Neurosurgical Site Infections after Craniotomy: A Prospective Multicenter Study of 2944 Patients. Neurosurgery. 1997;41:1073-1081.

397. Kourbeti IS, Vakis AF, Ziakas P, Karabetsos D, Potolidis E, Christou $\mathrm{S}$, et al. Infections in patients undergoing craniotomy: risk factors associated with post-craniotomy meningitis. J Neurosurg. 2014;122:1113-1119.

398. Inoue T, Shimizu H, Fujimura M, Sato K, Endo H, Niizuma K, et al. Risk factors for meningitis after craniotomy in patients with subarachnoid hemorrhage due to anterior circulation aneurysms rupture. Clin Neurol Neurosurg. 2015;139:302-306.

399. Chen C, Zhang B, Yu S, Sun F, Ruan Q, Zhang W, et al. The Incidence and Risk Factors of Meningitis after Major Craniotomy in China: A Retrospective Cohort Study. PLOS ONE. 2014;9:e101961.

400. Patir R, Mahapatra AK, Banerji AK. Risk factors in postoperative neurosurgical infection: A prospective study. Acta Neurochir (Wien). 1992;119:80-84.

401. Lietard C, Thébaud V, Besson G, Lejeune B. Risk factors for neurosurgical site infections: an 18-month prospective survey: Clinical article. J Neurosurg. 2008;109:729-34.

402. De Bels D, Korinek A-M, Bismuth R, Trystram D, Coriat P, Puybasset L. Empirical Treatment of Adult Postsurgical Nosocomial Meningitis. Acta Neurochir (Wien). 2002;144:989-995.

403. Chidambaram S, Nair MN, Krishnan SS, Cai L, Gu W, Vasudevan MC. Postoperative Central Nervous System Infection After Neurosurgery in a Modernized, ResourceLimited Tertiary Neurosurgical Center in South Asia. World Neurosurg. 2015;84:1668-1673.

404. Alotaibi AF, Hulou MM, Vestal M, Alkholifi F, Asgarzadeh M, Cote DJ, et al. The Efficacy of Antibacterial Prophylaxis Against the Development of Meningitis After Craniotomy: A Meta-Analysis. World Neurosurg. 2016;90:597-603.e1.

405. Moen V, Dahlgren N, Irestedt L. Severe Neurological Complications after Central Neuraxial Blockades in Sweden 1990-1999. Anesthesiol J Am Soc Anesthesiol. 2004;101: 950-959.
406. Baer ET. Post-dural puncture bacterial meningitis. Anesthesiology. 2006;105:381-93.

407. Baltas I, Tsoulfa S, Sakellariou P, Vogas V, Fylaktakis M, Kondodimou A. Posttraumatic MeningitisBacteriology, Hydrocephalus, and Outcome. Neurosurgery. 1994;35:422427.

408. Kourbeti IS, Vakis AF, Papadakis JA, Karabetsos DA, Bertsias $\mathrm{G}$, Filippou $\mathrm{M}$, et al. Infections in traumatic brain injury patients. Clin Microbiol Infect. 2012;18:359-364.

409. Ratilal BO, Costa J, Pappamikail L, Sampaio C. Antibiotic prophylaxis for preventing meningitis in patients with basilar skull fractures. Cochrane Database Syst Rev. 2015;CD004884.

410. Vinchon M, Dhellemmes P. Cerebrospinal fluid shunt infection: risk factors and long-term follow-up. Childs Nerv Syst. 2006;22:692-697.

411. Kulkarni AV, Drake JM, Lamberti-Pasculli M. Cerebrospinal fluid shunt infection: a prospective study of risk factors. J Neurosurg. 2001;94:195-201.

412. Tulipan N, Cleves MA. Effect of an intraoperative doublegloving strategy on the incidence of cerebrospinal fluid shunt infection. J Neurosurg. 2006;104:5-8.

413. Meyer CN, Samuelsson IS, Galle M, Bangsborg JM. Adult bacterial meningitis: aetiology, penicillin susceptibility, risk factors, prognostic factors and guidelines for empirical antibiotic treatment. Clin Microbiol Infect. 2004;10:709-717.

414. Erdem H, Elaldi N, Öztoprak N, Sengoz G, Ak O, Kaya S, et al. Mortality indicators in pneumococcal meningitis: therapeutic implications. Int J Infect Dis. 2014;19:13-19.

415. Prasad K, Kumar A, Singhal T, Gupta PK. Third generation cephalosporins versus conventional antibiotics for treating acute bacterial meningitis. Cochrane Database Syst Rev. 2007;CD001832.

416. Sarvepalli AK, Dharana PK. Clinical profile, bacterial profile and outcomes of acute bacterial meningitis in a tertiary care hospital- one year study. Int J Adv Med. 2017;4:502.

417. Tunkel AR, Hartman BJ, Kaplan SL, Kaufman BA, Roos KL, Scheld WM, et al. Practice Guidelines for the Management of Bacterial Meningitis. Clin Infect Dis. 2004;39:1267-1284.

418. Falagas ME, Bliziotis IA, Tam VH. Intraventricular or intrathecal use of polymyxins in patients with Gram-negative meningitis: a systematic review of the available evidence. Int J Antimicrob Agents. 2007;29:9-25.

419. Ng K, Mabasa VH, Chow I, Ensom MHH. Systematic Review of Efficacy, Pharmacokinetics, and Administration of Intraventricular Vancomycin in Adults. Neurocrit Care. 2014;20:158-171.

420. Tunkel AR, Hasbun R, Bhimraj A, Byers K, Kaplan SL, Scheld WM, et al. 2017 Infectious Diseases Society of America's Clinical Practice Guidelines for Healthcare-Associated Ventriculitis and Meningitis*. Clin Infect Dis. 2017;64:e34-65.

421. Zhang Z, Cai X, Li J, Kang X, Wang H, Zhang L, et al Retrospective analysis of 620 cases of brain abscess in Chinese patients in a single center over a 62-year period. Acta Neurochir (Wien). 2016;158:733-739.

422. Yang S-Y. Brain abscess: a review of 400 cases. J Neurosurg. 1981;55:794-799.

423. Brouwer MC, Coutinho JM, van de Beek D. Clinical characteristics and outcome of brain abscess: Systematic review and meta-analysis. Neurology. 2014;82:806-813.

424. Lakshmi V, Rao RR, Dinakar I. Bacteriology of brain abscess observations on 50 cases. J Med Microbiol. 1993;38: 187-190. 
425. Lakshmi V, Umabala P, Anuradha K, Padmaja K, Padmasree C, Rajesh A, et al. Microbiological Spectrum of Brain Abscess at a Tertiary Care Hospital in South India: 24-Year Data and Review. Pathol Res Int. 2011;2011:1-12.

426. Prasad KN, Mishra AM, Gupta D, Husain N, Husain M, Gupta RK. Analysis of microbial etiology and mortality in patients with brain abscess. J Infect. 2006;53:221-227.

427. Menon S, Bharadwaj R, Chowdhary A, Kaundinya DV, Palande DA. Current epidemiology of intracranial abscesses: a prospective 5 year study. J Med Microbiol. 2008;57:1259-1268.

428. Brouwer MC, Coutinho JM, van de Beek D. Clinical characteristics and outcome of brain abscess: systematic review and meta-analysis. Neurology. 2014;82:806-813.

429. Arlotti M, Grossi P, Pea F, Tomei G, Vullo V, De Rosa FG, et al. Consensus document on controversial issues for the treatment of infections of the central nervous system: bacterial brain abscesses. Int J Infect Dis. 2010;14:S79-92.

430. Angus DC, Linde-Zwirble WT, Lidicker J, Clermont G, Carcillo J, Pinsky MR. Epidemiology of severe sepsis in the United States: analysis of incidence, outcome, and associated costs of care. Crit Care Med. 2001;29:1303-1310.

431. Mayr FB, Yende S, Angus DC. Epidemiology of severe sepsis. Virulence. 2014;5:4-11.

432. Eron LJ. Managing skin and soft tissue infections: expert panel Recommendationss on key decision points. J Antimicrob Chemother. 2003;52:3i-17.

433. Stevens DL, Bisno AL, Chambers HF, Dellinger EP, Goldstein EJC, Gorbach SL, et al. Practice Guidelines for the Diagnosis and Management of Skin and Soft Tissue Infections: 2014 Update by the Infectious Diseases Society of America. Clin Infect Dis. 2014;59:e10-52.

434. Mohanty A, Mohapatra K, Pal B. Isolation and Identification of Staphylococcus aureus from Skin and Soft Tissue Infection in Sepsis Cases, Odisha. J Pure Appl Microbiol. 2018;12:419-424.

435. Zarrin Afroz JP. Bacteriological Profile and Antimicrobial Susceptibility Pattern of Skin and Soft Tissue Infections among Gram Negative Bacilli in a Tertiary Care Hospital of South India. J Pharm Sci. 2015;7:4

436. Mohanty S, Kapil A, Dhawan B, Das BK. Bacteriological and antimicrobial susceptibility profile of soft tissue infections from Northern India. Indian J Med Sci. 2004;58:10-15.

437. Joshi S, Ray P, Manchanda V, Bajaj J, Chitnis DS, Gautam V, et al. Methicillin resistant Staphylococcus aureus (MRSA) in India: Prevalence \& susceptibility pattern. Indian J Med Res. 2013;137:363-369.

438. Giuliano A, Lewis F, Hadley K, Blaisdell FW. Bacteriology of necrotizing fasciitis. Am J Surg. 1977;134:52-57.

439. Wang J-M, Lim H-K. Necrotizing fasciitis: eight-year experience and literature review. Braz J Infect Dis Off Publ Braz Soc Infect Dis. 2014;18:137-143.

440. Singh G, Ray P, Sinha SK, Adhikary S, Khanna SK. Bacteriology of necrotizing infections of soft tissues. Aust N Z J Surg. 1996;66:747-750.

441. Arabi YM, Dara SI, Tamim HM, Rishu AH, Bouchama A, Khedr $\mathrm{MK}$, et al. Clinical characteristics, sepsis interventions and outcomes in the obese patients with septic shock: an international multicenter cohort study. Crit Care Lond Engl. 2013;17:R72.

442. Shen H-N, Lu C-L. Skin and soft tissue infections in hospitalized and critically ill patients: a nationwide population-based study. BMC Infect Dis. 2010;10:151.

443. McClaine RJ, Husted TL, Hebbeler-Clark RS, Solomkin JS. Meta-Analysis of Trials Evaluating Parenteral Antimicrobial
Therapy for Skin and Soft Tissue Infections. Clin Infect Dis. 2010;50:1120-1126.

444. Neville LO, Brumfitt W, Hamilton-Miller JMT, Harding I. Teicoplanin $v s$. vancomycin for the treatment of serious infections: a randomised trial. Int J Antimicrob Agents. 1995;5: 187-193.

445. Liu C-Y, Lee W-S, Fung C-P, Cheng N-C, Liu C-L, Yang S-P, et al. Comparative Study of Teicoplanin vs Vancomycin for the Treatment of Methicillin-Resistant Staphylococcus aureus Bacteraemia. Clin Drug Investig. 1996;12:80-87.

446. Peng Y, Ye X, Li Y, Bu T, Chen X, Bi J, et al. Teicoplanin as an Effective Alternative to Vancomycin for Treatment of MRSA Infection in Chinese Population: AMeta-analysis of Randomized Controlled Trials. Schlievert PM, editor. PLoS ONE. 2013;8: e79782.

447. Davis SL, McKinnon PS, Hall LM, Delgado G, Rose W, Wilson $\mathrm{RF}$, et al. Daptomycin versus vancomycin for complicated skin and skin structure infections: clinical and economic outcomes. Pharmacotherapy. 2007;27:1611-1618.

448. White B, Seaton RA. Complicated skin and soft tissue infections: literature review of evidence for and experience with daptomycin. Infect Drug Resist. 2011;4:115-127.

449. Hepburn MJ, Dooley DP, Skidmore PJ, Ellis MW, Starnes WF, Hasewinkle WC. Comparison of short-course (5 days) and standard (10 days) treatment for uncomplicated cellulitis. Arch Intern Med. 2004;164:1669-1674.

450. Shankar-Hari M, Phillips GS, Levy ML, Seymour CW, Liu VX, Deutschman CS, et al. Developing a New Definition and Assessing New Clinical Criteria for Septic Shock: For the Third International Consensus Definitions for Sepsis and Septic Shock (Sepsis-3). JAMA. 2016;315:775-787.

451. Hatfield KM, Dantes RB, Baggs J, Sapiano MRP, Fiore $\mathrm{AE}$, Jernigan JA, et al. Assessing Variability in HospitalLevel Mortality Among U.S. Medicare Beneficiaries With Hospitalizations for Severe Sepsis and Septic Shock*. Crit Care Med. 2018;46:1753.

452. Kaukonen K-M, Bailey M, Suzuki S, Pilcher D, Bellomo R. Mortality Related to Severe Sepsis and Septic Shock Among Critically Ill Patients in Australia and New Zealand, 20002012. JAMA. 2014;311:1308.

453. Ferrer R, Martin-Loeches I, Phillips G, Osborn TM, Townsend S, Dellinger RP, et al. Empiric Antibiotic Treatment Reduces Mortality in Severe Sepsis and Septic Shock From the First Hour: Results From a Guideline-Based Performance Improvement Program*. Crit Care Med. 2014;42:1749-1755.

454. Dellinger RP, Schorr CA, Levy MM. A Users' Guide to the 2016 Surviving Sepsis Guidelines: Crit Care Med. 2017;45:381-385.

455. Kumar A, Zarychanski R, Light B, Parrillo J, Maki D, Simon D, et al. Early combination antibiotic therapy yields improved survival compared with monotherapy in septic shock: A propensitymatched analysis*: Crit Care Med. 2010;38:1773-1785.

456. Kumar A, Safdar N, Kethireddy S, Chateau D. A survival benefit of combination antibiotic therapy for serious infections associated with sepsis and septic shock is contingent only on the risk of death: A meta-analytic/meta-regression study: Crit Care Med. 2010;38:1651-1664.

457. Kullberg BJ, Arendrup MC. Invasive Candidiasis. Campion EW, editor. N Engl J Med. 2015;373:1445-56.

458. Vincent J-L. International Study of the Prevalence and Outcomes of Infection in Intensive Care Units. JAMA. 2009;302:2323. 
459. Horn DL, Neofytos D, Anaissie EJ, Fishman JA, Steinbach WJ, Olyaei AJ, et al. Epidemiology and Outcomes of Candidemia in 2019 Patients: Data from the Prospective Antifungal Therapy Alliance Registry. Clin Infect Dis. 2009;48:1695-1703.

460. McKinnon PS. Temporal Assessment of Candida Risk Factors in the Surgical Intensive Care Unit. Arch Surg. 2001;136:1401.

461. Michalopoulos AS, Geroulanos S, Mentzelopoulos SD. Determinants of candidemia and candidemia-related death in cardiothoracic ICU patients. Chest. 2003;124:2244-2255.

462. Muskett H, Shahin J, Eyres G, Harvey S, Rowan K, Harrison D. Risk factors for invasive fungal disease in critically ill adult patients: a systematic review. Crit Care. 2011;15:R287.

463. PC G, HK J. Routine versus selective antifungal administration for control of fungal infections in patients with cancer. 2014;50.

464. Cruciani M, de Lalla F, Mengoli C. Prophylaxis of Candida infections in adult trauma and surgical intensive care patients: a systematic review and meta-analysis. Intensive Care Med. 2005;31:1479-1487.

465. Patel TS, Eschenauer GA, Stuckey LJ, Carver PL. Antifungal Prophylaxis in Lung Transplant Recipients: Transplantation. 2016;100:1815-26.

466. Fekkar A, Dannaoui E, Meyer I, Imbert S, Brossas JY, Uzunov $\mathrm{M}$, et al. Emergence of echinocandin-resistant Candida spp. in a hospital setting: a consequence of 10 years of increasing use of antifungal therapy? Eur J Clin Microbiol Infect Dis. 2014;33:1489-1496.

467. Lortholary O, Desnos-Ollivier M, Sitbon K, Fontanet A, Bretagne S, Dromer F. Recent Exposure to Caspofungin or Fluconazole Influences the Epidemiology of Candidemia: a Prospective Multicenter Study Involving 2,441 Patients. Antimicrob Agents Chemother. 2011;55:532-538.

468. Pfaller MA. Antifungal Drug Resistance: Mechanisms, Epidemiology, and Consequences for Treatment. Am J Med. 2012;125:S3-13.

469. Garbino J, Lew D, Jacques-A. R, Hugonnet S, Auckenthaler $\mathrm{R}$, Pittet D. Prevention of severe Candida infections in nonneutropenic, high-risk, critically ill patients: a randomized, double-blind, placebo-controlled trial in patients treated by selective digestive decontamination. Intensive Care Med. 2002;28:1708-1717.

470. RexJH,Pappas PG, Karchmer AW, Sobel J, EdwardsJE, Hadley S, et al. A Randomized and Blinded Multicenter Trial of High-Dose Fluconazole plusPlaceboversus Fluconazole plus Amphotericin B as Therapy for Candidemia and Its Consequences in Nonneutropenic Subjects. Clin Infect Dis. 2003;36:1221-8.

471. Ostrosky-Zeichner L, Al-Obaidi M. Invasive Fungal Infections in the Intensive Care Unit. Infect Dis Clin North Am. 2017;31:475-487.

472. Namikawa T, Kitagawa H, Yamatsuji T, Naomoto Y, Kobayashi M, Hanazaki K. Pre-emptive treatment of fungal infection based on plasma $\beta$-D-glucan levels after gastric surgery for gastric cancer in elderly patients: Pre-emptive therapy for fungal infection. J Gastroenterol Hepatol. 2013;28:1457-1461.

473. Timsit J-F, Azoulay E, Schwebel C, Charles PE, Cornet M, Souweine B, et al. Empirical Micafungin Treatment and Survival Without Invasive Fungal Infection in Adults With ICU-Acquired Sepsis, Candida Colonization, and Multiple Organ Failure: The EMPIRICUS Randomized Clinical Trial. JAMA. 2016;316:1555.
474. Cortegiani A, Russotto V, Maggiore A, Attanasio M, Naro AR Raineri SM, et al. Antifungal agents for preventing fungal infections in non-neutropenic critically ill patients. Cochrane Database Syst Rev. 2016;CD004920.

475. Mathur P, Gunjiyal J, Tak V, Varghese P, Xess I, Misra M. The epidemiological profile of candidemia at an Indian trauma care center. J Lab Physicians. 2014;6:96.

476. Society for Healthcare Epidemiology of America, Infectious Diseases Society of America, Pediatric Infectious Diseases Society. Policy statement on antimicrobial stewardship by the Society for Healthcare Epidemiology of America (SHEA), the Infectious Diseases Society of America (IDSA), and the Pediatric Infectious Diseases Society (PIDS). Infect Control Hosp Epidemiol. 2012;33:322-327.

477. Tamma PD, Avdic E, Li DX, Dzintars K, Cosgrove SE. Association of Adverse Events With Antibiotic Use in Hospitalized Patients. JAMA Intern Med. 2017;177:1308-1315.

478. Centers for Disease Control. Core Elements of Hospital Antibiotic Stewardship Programs Antibiotic Use. 2017.

479. Schuts EC, Hulscher MEJL, Mouton JW, Verduin CM, Stuart JWTC, Overdiek HWPM, et al. Current evidence on hospital antimicrobial stewardship objectives: a systematic review and meta-analysis. Lancet Infect Dis. 2016;16:847-856.

480. Tabah A, Cotta MO, Garnacho-Montero J, Schouten J, Roberts JA, Lipman J, et al. A Systematic Review of the Definitions, Determinants, and Clinical Outcomes of Antimicrobial Deescalation in the Intensive Care Unit. Clin Infect Dis Off Publ Infect Dis Soc Am. 2016;62:1009-1017.

481. Leone M, Bechis C, Baumstarck K, Lefrant J-Y, Albanèse J, Jaber S, et al. De-escalation versus continuation of empirical antimicrobial treatment in severe sepsis: a multicenter non-blinded randomized noninferiority trial. Intensive Care Med. 2014;40:1399-1408.

482. Davey P, Marwick CA, Scott CL, Charani E, McNeil K, Brown $\mathrm{E}$, et al. Interventions to improve antibiotic prescribing practices for hospital inpatients. In: Cochrane Database of Systematic Reviews [Internet]. John Wiley \& Sons, Ltd; 2017.

483. Barlam TF, Cosgrove SE, Abbo LM, MacDougall C, Schuetz AN, Septimus EJ, et al. Implementing an Antibiotic Stewardship Program: Guidelines by the Infectious Diseases Society of America and the Society for Healthcare Epidemiology of America. Clin Infect Dis. 2016;62:e51-77.

484. Dellit TH, Owens RC, McGowan JE, Gerding DN, Weinstein RA, Burke JP, et al. Infectious Diseases Society of America and the Society for Healthcare Epidemiology of America Guidelines for Developing an Institutional Program to Enhance Antimicrobial Stewardship. Clin Infect Dis. 2007;44:159-177.

485. Cosgrove SE, Seo SK, Bolon MK, Sepkowitz KA, Climo MW, Diekema DJ, et al. Evaluation of postprescription review and feedback as a method of promoting rational antimicrobial use: a multicenter intervention. Infect Control Hosp Epidemiol. 2012;33:374-380.

486. Vettese N, Hendershot J, Irvine M, Wimer S, Chamberlain D, Massoud N. Outcomes associated with a thrice-weekly antimicrobial stewardship programme in a 253-bed community hospital. J Clin Pharm Ther. 2013;38:401-404.

487. Tamma PD, Avdic E, Keenan JF, Zhao Y, Anand G, Cooper J, et al. What Is the More Effective Antibiotic Stewardship Intervention: Preprescription Authorization or Postprescription Review With Feedback? Clin Infect Dis Off Publ Infect Dis Soc Am. 2017;64:537-543.

488. Mehta JM, Haynes K, Wileyto EP, Gerber JS, Timko DR, Morgan SC, et al. Comparison of Prior Authorization 
and Prospective Audit with Feedback for Antimicrobial Stewardship. Infect Control Hosp Epidemiol. 2014;35: 1092-1099.

489. Kollef MH, Vlasnik J, Sharpless L, Pasque C, Murphy D, Fraser V. Scheduled change of antibiotic classes: a strategy to decrease the incidence of ventilator-associated pneumonia. Am J Respir Crit Care Med. 1997;156:1040-1048.

490. Gruson D, Hilbert G, Vargas F, Valentino R, Bebear C, Allery A, et al. Rotation and restricted use of antibiotics in a medical intensive care unit. Impact on the incidence of ventilator-associated pneumonia caused by antibioticresistant gram-negative bacteria. Am J Respir Crit Care Med. 2000;162:837-843.

491. Raymond DP, Pelletier SJ, Crabtree TD, Gleason TG, Hamm LL, Pruett TL, et al. Impact of a rotating empiric antibiotic schedule on infectious mortality in an intensive care unit. Crit Care Med. 2001;29:1101-1108.

492. Cobos-Trigueros N, Solé M, Castro P, Torres JL, Rinaudo M, De Lazzari E, et al. Evaluation of a Mixing versus a Cycling Strategy of Antibiotic Use in Critically-Ill Medical Patients: Impact on Acquisition of Resistant Microorganisms and Clinical Outcomes. PloS One. 2016;11:e0150274.

493. Jones M, Huttner B, Madaras-Kelly K, Nechodom K, Nielson C, Goetz MB, et al. Parenteral to Oral Conversion of Fluoroquinolones: Low-Hanging Fruit for Antimicrobial Stewardship Programs? Infect Control Hosp Epidemiol. 2012;33:362-367.

494. Sevinç F, Prins JM, Koopmans RP, Langendijk PN, Bossuyt PM, Dankert J, et al. Early switch from intravenous to oral antibiotics: guidelines and implementation in a large teaching hospital. J Antimicrob Chemother. 1999;43:601-606.
495. Mertz D, Koller M, Haller P, Lampert ML, Plagge H, Hug B, et al. Outcomes of early switching from intravenous to oral antibiotics on medical wards. J Antimicrob Chemother. 2009;64:188-199.

496. Oosterheert JJ, Bonten MJM, Schneider MME, Buskens E, Lammers J-WJ, Hustinx WMN, et al. Effectiveness of early switch from intravenous to oral antibiotics in severe community acquired pneumonia: multicentre randomised trial. BMJ. 2006;333:1193.

497. Omidvari K, de Boisblanc BP, Karam G, Nelson S, Haponik E, Summer W. Early transition to oral antibiotic therapy for community-acquired pneumonia: duration of therapy, clinical outcomes, and cost analysis. Respir Med. 1998;92:1032-1039.

498. van Niekerk AC, Venter DJL, Boschmans S-A. Implementation of intravenous to oral antibiotic switch therapy guidelines in the general medical wards of a tertiary-level hospital in South Africa. J Antimicrob Chemother. 2012;67:756-762.

499. Guo Y, Gao W, Yang H, Ma C' 'en, Sui S. De-escalation of empiric antibiotics in patients with severe sepsis or septic shock: A meta-analysis. Heart Lung J Crit Care. 2016;45:454-459.

500. Westwood M, Ramaekers B, Whiting P, Tomini F, Joore M, Armstrong N, et al. Procalcitonin testing to guide antibiotic therapy for the treatment of sepsis in intensive care settings and for suspected bacterial infection in emergency department settings: a systematic review and cost-effectiveness analysis. Health Technol Assess Winch Engl. 2015;19:v-xxv, 1-236.

501. Jong E de, Oers JA van, Beishuizen A, Vos P, Vermeijden WJ, Haas LE, et al. Efficacy and safety of procalcitonin guidance in reducing the duration of antibiotic treatment in critically ill patients: a randomised, controlled, open-label trial. Lancet Infect Dis. 2016;16:819-827. 